UCRL-TR-203529

Document UCRL-TR-203529

This document contains $\underline{64}$ pages.

\title{
Neutrons and Granite: Transport and Activation
}

\author{
Peter J. Bedrossian
}

April 2004

Lawrence Livermore National Laboratory

Livermore CA 94551

Work was performed at Lawrence Livermore National Laboratory under the auspices of the University of California under US-DOE Contract W-7405-Eng-48. 
This document was prepared as an account of work sponsored by an agency of the United States Government. Neither the United States Government nor the University of California nor any of their employees, makes any warranty, express or implied, or assumes any legal liability or responsibility for the accuracy, completeness, or usefulness of any information, apparatus, product, or process disclosed, or represents that its use would not infringe privately owned rights. Reference herein to any specific commercial product, process, or service by trade name, trademark, manufacturer, or otherwise, does not necessarily constitute or imply its endorsement, recommendation, or favoring by the United States Government or the University of California. The views and opinions of authors expressed herein do not necessarily state or reflect those of the United States Government or the University of California, and shall not be used for advertising or product endorsement purposes. 
UCRL-TR-203529

This page intentionally left blank to facilitate double-sided printing 


\begin{abstract}
In typical ground materials, both energy deposition and radionuclide production by energetic neutrons vary with the incident particle energy in a non-monotonic way. We describe the overall balance of nuclear reactions involving neutrons impinging on granite to demonstrate these energy-dependencies. While granite is a useful surrogate for a broad range of soil and rock types, the incorporation of small amounts of water (hydrogen) does alter the balance of nuclear reactions.
\end{abstract}


UCRL-TR-203529

This page intentionally left blank to facilitate double-sided printing. 


\section{Table of Contents}

Abstract .




\section{List of Tables}

Table 1: Composition of simplified granite used in this study. The calculations reflect the natural isotopic abundances for each element.............................................. 34

Table 2: Radionuclides included in published lists of contaminant nuclides which may be created by neutron irradiation of ground. Those nuclides shown in red are radioactive. Note that not all of these radionuclides can be produced from the isotopes included in the simplified granite of Table 1, but they may be produced by neutron reactions with trace elements............................................................... 35

Table 3: Available cross-sections for producing each of the radionuclides listed in Table

2. The reactions included in calculations described in this work are highlighted in yellow. Those which are not highlighted involve target nuclides which are not included in the simplified granite formulation considered here .......................... 36

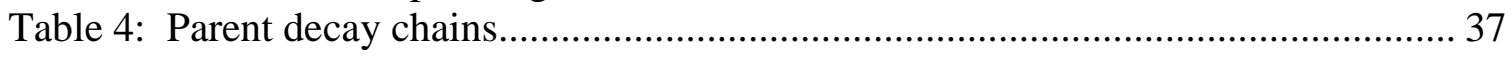


UCRL-TR-203529

\section{List of Figures}

Figure 1: Test problem geometry.

20

Figure 2: Calculated cumulative energy deposition into granite from monoenergetic, planar neutron sources with incident angles as indicated. Note zero suppression on

the vertical scale.

Figure 3: Calculated cumulative energy deposition into granite and into the principal atomic components of granite individually from monoenergetic, planar neutron sources and normal incidence.

Figure 4: Calculated cumulative energy deposition into granite by depth. This and all subsequent plots involve neutrons at normal incidence.

Figure 5: Calculated ${ }^{16} \mathrm{O}$ (n,elastic) reactions in granite by depth for normal incidence.. 22

Figure 6: Calculated ${ }^{28} \mathrm{Si}(\mathrm{n}$,elastic) reactions in granite by depth. ............................... 23

Figure 7: Calculated inelastic reactions of neutrons and ${ }^{16} \mathrm{O}$ in granite by depth............ 23

Figure 8: Calculated inelastic reactions of neutrons and ${ }^{28} \mathrm{Si}$ in granite by depth.......... 24

Figure 9: Calculated ${ }^{16} \mathrm{O}(\mathrm{n}, \alpha)$ reactions in granite by depth......................................... 24

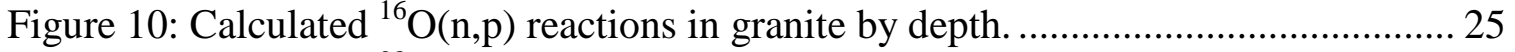

Figure 11: Calculated ${ }^{28} \mathrm{Si}(\mathrm{n}, \alpha)$ reactions in granite by depth. ......................................2. 25

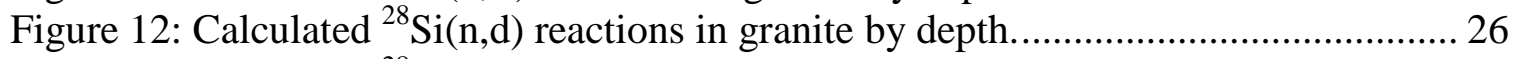

Figure 13: Calculated ${ }^{28} \mathrm{Si}(\mathrm{n}, \gamma)$ reactions in granite by depth...................................... 26

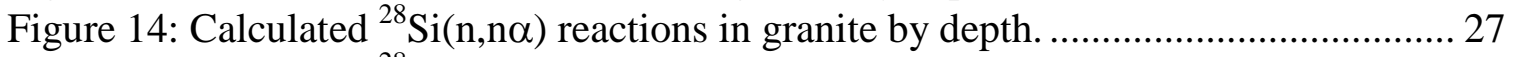

Figure 15: Calculated ${ }^{28} \mathrm{Si}(\mathrm{n}, \mathrm{p})$ reactions in granite by depth..................................... 27

Figure 16: Calculated ${ }^{28} \mathrm{Si}(\mathrm{n}, \mathrm{np})$ reactions in granite by depth..................................... 28

Figure 17: Calculated energy deposition by depth into the ${ }^{16} \mathrm{O}$ component of granite only.

Other components are removed from the problem. ............................................... 29

Figure 18: Calculated, total neutron reactions by depth into the ${ }^{16} \mathrm{O}$ component of granite

only. Other components are removed from the problem.................................... 29

Figure 19: Calculated energy deposition by depth into the ${ }^{28} \mathrm{Si}$ component of granite only.

Other components are removed from the problem. .......................................... 30

Figure 20: Calculated, total neutron reactions by depth into the ${ }^{28} \mathrm{Si}$ component of granite

only. Other components are removed from the problem.................................... 30

Figure 21: Summary of the dominant reactions for neutrons incident on granite.

Reactions involving ${ }^{16} \mathrm{O}$ are represented with solid curves, while reactions involving

${ }^{28} \mathrm{Si}$ are represented with dashed curves.......................................................... 31

Figure 22: Calculated energy deposition by depth in granite vs. percentage of added

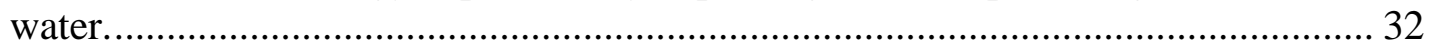

Figure 23: Effects of added water on neutron reactions in granite. ............................... 32

Figure 24: Total production of specific radioisotopes in granite from planar,

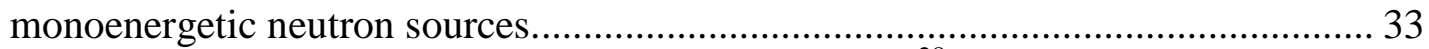

Figure 25: (Appendix A) Neutron cross-sections for target ${ }^{28} \mathrm{Si}$ from ENDF/B-VI.8.... 38 Figure 26: (Appendix A) Total Cross Section for neutrons incident on ${ }^{28} \mathrm{Si}$, from ENDF/B-VI.8 
Figure 27: (Appendix A) Neutron cross-sections for target ${ }^{16} \mathrm{O}$ from ENDF/B-VI.8 ..... 40

Figure 28: (Appendix A) Neutron cross-sections for target ${ }^{16} \mathrm{O}$ from ENDL99. .............. 41

Figure 29: (Appendix A) Total Cross Section for neutrons incident on ${ }^{16} \mathrm{O}$, from both ENDF/B-VI.8 and ENDL99.

Figure 30: (Appendix A) ENDF/B-VI cross sections for dominant transmutation reactions in granite, WEIGHTED BY THE ATOMIC COMPOSITIONS IN

GRANITE. The vertical scale is the cross section multiplied by the number of atoms/barn-cm of the target nuclide.

Figure 31: (Appendix A) Neutron elastic cross-sections for ${ }^{1} \mathrm{H},{ }^{16} \mathrm{O}$, and ${ }^{28} \mathrm{Si} . \ldots \ldots \ldots \ldots \ldots . . . . . .44$

Figure 32: (Appendix A) Capture cross-sections for ${ }^{1} \mathrm{H},{ }^{16} \mathrm{O}$, and ${ }^{28} \mathrm{Si}$........................ 45 


\section{Introduction}

The release of energetic neutrons in the vicinity of the earth's surface, as might occur in a nuclear explosion, will affect the ground in two ways: (i) collisions and nuclear reactions will transfer energy from the incident neutrons to the ground, resulting in ground heating that can lead to melting, vaporization, and shock generation; and (ii) neutron reactions can generate hazardous radionuclides. ${ }^{1}$ Trace elements present in soil can be activated to generate non-negligible quantities of radioisotopes. Mitigation of collateral effects from the release of energetic neutrons requires a detailed understanding of the interaction of neutrons with the ground. The methodology described here provides a tool to evaluate collateral damage from radioisotope activation for specific conditions of neutron release, ground composition, and terrain. While this study uses simple models that capture the essential physics, the methodology is easily generalizable to a broad range of general neutron sources, ground compositions, and details of terrain.

Specifically, we consider a planar, monoenergetic neutron source at a height of $1 \mathrm{~m}$ above granite. (Figure 1) Granite is a natural surrogate for many soils and rocks because it is composed primarily of $\mathrm{SiO}_{2}$, and its composition closely approximates that of the earth's crust. Apart from Limestone, the more common soils and rocks on the earth's surface are composed primarily of Si and O. [1] Using MCNP, we calculate the energy deposition as a function of depth in the ground and correlate the energy deposition with neutron reactions taking place for each incident neutron energy. The production of hazardous radionuclides is included in the calculations. Finally, we consider small variations in granite composition.

\footnotetext{
${ }^{1}$ C. Bridgman, The Effects of Nuclear Weapons (VA: DTRA, 2001).
} 


\section{Method}

All of the calculations described below used Version 5 of the MCNP code. A comprehensive description of the capabilities of the code and the underlying physics is provided in the documentation for the code. ${ }^{2}$ Each calculation uses a planar source of monoenergetic neutrons of radius $10 \mathrm{~m}$, located $1 \mathrm{~m}$ above the surface of the ground. The total problem radius within which neutrons and neutron-induced gammas are tallied is typically $100 \mathrm{~m}$. The source neutrons are all directed toward the ground, either at normal incidence or at a specified angle. The ground is partitioned into flat slabs with logarithmically-spaced boundaries parallel to the flat surface. The calculations were typically started with 20,000 census particles and with biasing such that particle importance increased with depth into the ground. We have used a simplified granite composition for the ground, given in Table $1 .^{3}$ For reference we have included in the table the concentrations of various elements in the earth's crust, and in pure $\mathrm{SiO}_{2}$. Because the dominant constituents of granite correspond generally to those in the earth's crust, the physics of neutron transport in granite may be expected to apply generally to a variety of soils and rocks with similar compositions. The model captures the essential features of the energy-dependence of neutron-ground coupling and demonstrates the calculation of radionuclide production rates. It can also be generalized easily to specific environments of interest.

All results are presented below in a normalized form and can therefore be scaled to arbitrary source strength. For example, energy deposition and reaction tallies are both normalized to the total incident energy fluence (number of source neutrons $\times$ energy per neutron), while radionuclide production calculations are normalized to the number of incident neutrons.

The Monte Carlo transport calculations described below use cross-sections from Release 8 of the ENDF/B-VI library where available. This is the most current library of

\footnotetext{
2 "MCNP_A General Monte Carlo N-Particle Transport Code, Version 5," LANL document LA-UR-03-1987. (2003).

${ }^{3}$ Source: T. S. Carman, private communication.
} 
evaluated cross-sections included wih Release 5 of MCNP. If no cross-section set is available in an ENDF library for a particular nuclide, then we use the ENDL-92 library. The latter is the most current release of an ENDL library which is available for use with MCNP.

In order to calculate radionuclide activation, we set up tallies for each reaction channel which might yield a particular radionuclide for which cross-sections are available. In this study we consider the hazardous radionuclides which are listed in either one of two standard lists. ${ }^{4,5}$ The consolidated list of hazardous radionuclides is presented in Table 2 below. The isotopes on the list fall into two categories: those with shorter half-lives (minutes, hours) pose a direct radiation hazard until they are depleted by radioactive decay, while the longer-lived isotopes can enter the food chain and become incorporated in biological tissue. While this is not a comprehensive list of all radionuclides which may be produced by neutron activation of soil, it is representative of the two, broad classes of hazardous isotopes, and the methodology below is easily generalizable to include other isotopes as needed for specific cases. ${ }^{6}$

In order to calculate the number of radionuclides of a particular species produced in reactions, it is necessary to add the contributions from any reactions that might produce that nuclide. In general, the evaluated cross-sections for those reactions may be scattered among different libraries. Appendix G of the MCNP manual [2] lists the target nuclides included in each cross-section library accessible to MCNP but not the specific reactions included in each library for a particular target. Lists of specific reactions are available for some evaluated cross-section libraries. ${ }^{7}$ However, the specific task of locating crosssections for each reaction that might generate a particular nuclide requires a backward

\footnotetext{
${ }^{4}$ John Northrop, ed., Handbook of Nuclear Weapon Effects, (Alexandria, Virginia: Defense Special Weapons Agency, 1996), p. 8-22.

${ }^{5}$ E. Teller, W. Talley, G. Higgins, and G. Johnson, The Constructive Uses of Nuclear Explosives (New York: McGraw-Hill, 1968), p, 107.

${ }^{6}$ Isotope data cited in the tables are derived from R. Firestone and V. Shirley, eds, Table of Isotopes, (John Wiley, 1999).

${ }^{7}$ See, for example, <<http://nuclear.1lnl.gov 〉>.
} 
directory which would provide targets, reactions, and source libraries for a given product nuclide. Because no such directory is generally available, we present one in Appendix C below. The table is generally applicable to any trace elements for which evaluated crosssections are available. Only radioactive product nuclei are represented in that table. If more than one library contains a cross-section for a particular reaction, we select a crosssection for the purpose of this study according to the following heirarchy: (i) ENDF/B$\mathrm{IV}^{8,9}$, (ii) ENDL92 ${ }^{10}$, (iii) ACTL and (iv) 532dos. The first contains the most up-to-date evaluations and therefore represents the most current experimental data. Either of the first two libraries may be used for transport calculations. The third and fourth libraries on the list are vintage 1970-1980 libraries from LLNL and LANL respectively which may be used only for dosimetry, not transport. Neither is actively maintained at this time, for many relevant target isotopes they hold the only accessible, evaluated cross-section data available.

Appendix C offers a complete directory of evaluated cross-sections available for use with MCNP for the production of radioisotopes. Using that table, we present in Table 3 a list of the available cross-sections for production of the radionuclides listed in Table 2, with source libraries selected on the basis of the hierarchy given above. Of the reactions listed in Table 3, those which are highlighted are included in the calculations represented in this work. Those which are not highlighted involve target nuclei which are not included in the simplified granite formulation used for this study.

In principle a complete accounting of radionuclide production should include not only generation by direct neutron irradiation but also generation by decay of parent nuclei. Table 4 presents an accounting of possible parent nuclei for each of the radionuclides listed in Table 2. According to Appendix C, production cross-sections

\footnotetext{
${ }^{8}$ J. M. Campbell, S. Frankle, and R. Little, "ENDF66: A continuous-Energy Neutron Data Library based on ENDF/B-VI Release 6," LANL Document LA-UR-03-0954.

9 J. Hendricks, S. Frankle, and J. Court, "ENDF/B-VI Data for MCNP," LANL Document LA-12891 (1994).

${ }^{10}$ S. Frankle, "Summary Documentation for the ENDL92 Continuous-Energy Neutron Data Library," LANL Document LA-UR-96-327 (1996)
} 
exist only for those few parent nuclei which are highlighted in Table 4. This component of radionuclide production is not included in the work described below.

We emphasize that this study is limited to the transport and reactions of neutrons and neutron-induced gammas. Other processes which might be consequences of energy deposition in the ground, such as hydrodynamics and material phase or strength evolution are beyond the scope of this work and should be addressed separately. 


\section{Results and Discussion}

\section{Neutron Transport and Energy Deposition in Dry Granite}

Because the principal atomic components of granite are $\mathrm{Si}$ and $\mathrm{O}$, we concentrate on the interaction of incident neutrons with each of those species. We shall show that combination of the results of neutron transport calculations for these two elements individually accounts for the essential features of neutron transport and energy deposition in granite. Because $\mathrm{Si}$ and $\mathrm{O}$ are also the dominant elemental species in most soils and rocks, with the exception of limestone, the results are applicable more generally.

The following discussion makes frequent reference to plots of evaluated crosssections in Appendix A. Figures 25 and 26 present the the evaluated cross-sections used in this study for ${ }^{28} \mathrm{Si}$, while figures $27-29$ present the evaluated cross-sections for ${ }^{16} \mathrm{O}$ targets. Figure 30 presents plots of the dominant transmutation reactions involving neutrons in granite, weighted by the concentration of each atomic species in the simplified granite formulation used in this work.

Figure 2 shows that the total energy deposition, normalized to the total source energy, for monoenergetic neutrons normally incident on granite rises strongly between incident energies of 1 and $2 \mathrm{MeV}$. As the source particle energy rises, the energy deposition falls to a minimum near $6 \mathrm{MeV}$, then rises, peaks again near $9 \mathrm{MeV}$, then falls off at higher energies. As the angle of incidence increases, the total energy deposition falls for all incident particle energies, but it falls slightly more strongly at lower energies $(<7 \mathrm{MeV})$. We shall show that the dependence of energy deposition on incident angle arises because lower-energy neutrons have a higher incidence of near-surface collisions which increase the likelihood of reflection and escape into the air.

In Figure 3, the energy deposition of incident neutrons is considered separately for ${ }^{16} \mathrm{O}$ and ${ }^{28} \mathrm{Si}$, the two dominant nuclides in granite. Oxygen and silicon respond differently to incident neutrons at any given, incident energy. Only the oxygen target shows the sharp rise in energy deposition between 1 and $2 \mathrm{MeV}$. Above $2 \mathrm{MeV}$, the oxygen target shows a decrease in energy deposition and a minum at $6 \mathrm{MeV}$, then rises 
between 6 and $8 \mathrm{MeV}$ and falls off at higher energies. The silicon target shows a peak in energy deposition near $3 \mathrm{MeV}$, then decreases to a minimum near $8 \mathrm{MeV}$.

Figure 4 presents the depth profiles of energy deposition in granite from monoenergetic surface sources. This and all subsequent figures reflect the results of calculations with neutrons at normal incidence. The lowest-energy neutrons $(1-2 \mathrm{MeV})$ deposit a higher proportion of their energy near the surface than do higher-energy neutrons.

${ }^{16} \mathrm{O}$ is characterized by a very low cross-section for neutron capture across the range of incident particle energies considered in this work. (Figure 27) For the lowest incident neutron eneries considered here, elastic scattering dominates. At higher energies, the $(\mathrm{n}, \alpha)$ channel opens first. In contrast, ${ }^{28} \mathrm{Si}$ has non-negligible capture cross-sections throughout the range of incident particle energies considered here (Figure 25), and inelastic neutron scattering off ${ }^{28} \mathrm{Si}$ has a significant cross-section at lower energies than for ${ }^{16} \mathrm{O}$.

Individual reaction tallies from MCNP calculations illustrate the consequences of these details of the ${ }^{16} \mathrm{O}$ and ${ }^{28} \mathrm{Si}$ neutron cross-sections. For the lowest incident neutron energies, elastic scattering off ${ }^{16} \mathrm{O}$ dominates. (Figure 5 and Figure 6) Inelastic scattering from ${ }^{16} \mathrm{O}$ is significant only for incident energies above $6 \mathrm{MeV}$ (Figure 7), but inelastic scattering from ${ }^{28} \mathrm{Si}$ is significant for incident energies $\geq 2 \mathrm{MeV}$ (Figure 8). For $1 \mathrm{MeV}$ incident neutrons, elastic scattering from ${ }^{16} \mathrm{O}$ leads to significant reflection from the surface. The ${ }^{16} \mathrm{O}(\mathrm{n}, \alpha)$ reaction channel, which is endothermic, opens above $2 \mathrm{MeV}$. (Figure 9) The energy deposition into ${ }^{16} \mathrm{O}$ falls to a local minimum near $6 \mathrm{MeV}$ but rises at higher energies as inelastic scattering becomes significant. Another endothermic channel associated with the ${ }^{16} \mathrm{O}(\mathrm{n}, \mathrm{p})$ reaction opens above $10 \mathrm{MeV}$ and leads to another decrease in energy deposition for incident particles exceeding that energy.

Because of the non-negligible capture cross section for $1 \mathrm{MeV}$ neutrons on ${ }^{28} \mathrm{Si}$, absorption of neutrons by ${ }^{28} \mathrm{Si}$ in granite is significant but small relative to elastic scattering over all incident neutron energies (Figure 13), and appreciable energy is still absorbed by the silicon target in the absence of oxygen even for $1 \mathrm{MeV}$ incident neutrons. (Figure 3). The dip in energy deposition for the silicon target in Figure 3 is associated with two threshold reactions, the ${ }^{28} \mathrm{Si}(\mathrm{n}, \mathrm{a})$ reaction (Figure 11) and of the ${ }^{28} \mathrm{Si}(\mathrm{n}, \mathrm{p})$ 
reaction (Figure 15) The opening of other endothermic reaction channels for ${ }^{28} \mathrm{Si}$ at higher still higher energies (Figure 12, Figure 14, and Figure 16) is associated with decreases in the energy deposition by incident neutrons at the highest energies considered here.

Energy deposition and neutron reactions for ${ }^{16} \mathrm{O}$ alone are summarized in Figure 17 and Figure 18 respectively, and for ${ }^{28} \mathrm{Si}$ in Figure 19 and Figure 20 respectively. For $1 \mathrm{MeV}$ neutrons incident on ${ }^{16} \mathrm{O}$ with the density of oxygen in granite, most energy deposition takes place within $50 \mathrm{~cm}$ of the surface. For incident energies $\geq 3 \mathrm{MeV}$ most energy deposition takes place within $1.8 \mathrm{~m}$ of the surface. In contrast, significant energy deposition into ${ }^{28} \mathrm{Si}$ with the density of silicon in granite takes place at depths exceeding $10 \mathrm{~m}$ for all incident energies.

A coherent picture for the transport and energy deposition of incident neutrons in granite emerges from these considerations. Figure 21 shows that elastic scattering from ${ }^{16} \mathrm{O}$ dominates elastic scattering from ${ }^{28} \mathrm{Si}$ for all incident neutron energies. At the lowest energies the ${ }^{28} \mathrm{Si}$ absorption reaction is dominated by elastic scattering from ${ }^{16} \mathrm{O}$. Therefore, lower-energy neutrons are confined near the surface by collisions, and the lowest-energy neutrons considered here $(\mathrm{MeV})$ are most likely to be reflected back to air and deposit the lowest proportion of their energy in the granite. Other features of the deposition profile for normal incidence (red curve in Figure 2 and Figure 3) are predominantly consequences of the competition among different reaction channels in ${ }^{16} \mathrm{O}$ and ${ }^{28} \mathrm{Si}$ with various cross-sections and threshold energy as described above.

We note here the importance of treating all reactions for a given target nuclide with a consistent cross-section library. Figure 27 and Figure 28 presat the evaluated cross sections for neutron reacctions in the ENDF/B-VI and ENDL99 libraries, respectively. While the total cross-sections from the two libraries are very close (Figure 29), the individual cross-sections differ because the two libraries may partition individual physical processes among the various cross-sections in different ways. For example, ENDL99 defines a ${ }^{16} \mathrm{O}(\mathrm{n}, \mathrm{n} \alpha)$ reaction. ENDF/B-VI instead folds that reaction into the cross-section for the inelastic reaction, ${ }^{16} \mathrm{O}\left(\mathrm{n}, \mathrm{n}^{\prime}\right)$, and treats the ${ }^{16} \mathrm{O}(\mathrm{n}, \mathrm{n} \alpha)$ reaction as two separate physical processes: (i) inelastic neutron scattering and promotion of the oxygen nuclide to an excited state, and (ii) alpha-decay of the excided state of ${ }^{16} \mathrm{O}$ to ${ }^{12} \mathrm{C}$, which 
is not included in scope of MCNP and is therefore not included in this work. The different shapes of the ${ }^{16} \mathrm{O}\left(\mathrm{n}, \mathrm{n}^{\prime}\right)$ cross-section curves from the two libraries above the ${ }^{16} \mathrm{O}(\mathrm{n}, \mathrm{n} \alpha)$ threshold reflect the different treatments of this process.

\section{Effect of composition variations}

The calculations described above are easily generalized for variations in ground composition. As an example we consider the effect of water inclusion in granite. Figure 31 shows that the elastic cross-sections for ${ }^{1} \mathrm{H},{ }^{16} \mathrm{O}$, and ${ }^{28} \mathrm{Si}$ are comparable in magnitude over the range of incident neutron energies considered in this work. Figure 32 shows that the capture cross-sections for ${ }^{1} \mathrm{H}$ and ${ }^{28} \mathrm{Si}$ are comparable in magnitude over the same range of neutron energies, except for resonances in the ${ }^{28} \mathrm{Si}$ capture cross-section. Figure 22 shows the energy deposition into granite from $14 \mathrm{MeV}$ incident neutrons increases monotonically with increasing water concentration from $68 \%$ to $72 \%$ of the total source energy for realistic variations in water content up through 5 weight percent. Figure 23 displays the variation of the two processes most susceptible to changing water content: elastic scattering and neutron capture. The occurrence of both processes per incident neutron energy fluence rises monotonically with water content for ${ }^{1} \mathrm{H}$ target nuclei and falls monotonically for ${ }^{16} \mathrm{O}$ and ${ }^{28} \mathrm{Si}$ target nuclei in the granite. We have seen above that elastic scattering from ${ }^{16} \mathrm{O}$ tended to promote reflection of neutrons and therefore to supress energy deposition in the granite. However, the close mass match of the incident neutrons and the ${ }^{1} \mathrm{H}$ nuclei would tend to impart more kinetic energy to the hydrogen nuclei than to oxygen. Therefore, enhancement of elastic scattering from hydrogen at the expense of scattering from oxygen would be expected to enhance overall energy deposition in the granite.

\section{Generation of hazardous radioisotopes}

The radionuclides produced by neutron activation of ground constituents fall into two categories, each of which presents a different hazard. The short-lived isotopes, with half-lives of the order of hours and minutes, present a direct radiation hazard while they remain radioactive. Isotopes with longer half-lives can enter the food chain and therefore present a longer-term hazard. Representatives of both classes are included in the 
calculations. In this work we calculate only the production of radioisotopes which can be generated by neutron irradiation of the simplified granite formulation. Other nuclides can be generated by neutron irradiation of trace elements, which can be added to calculations for specific environments as appropriate.

The figures in Appendix B present plots of all of the evaluated cross-sections available for the reactions represented in Table 3, organized by product nuclide. Figure 24 presents the results of MCNP calculations for the generation of each of the hazardous radioisotopes for which cross-sections involving the target nuclei in our simplified granite are available in the standard libraries. The energy-dependence of production of each nuclide is generally a result of the types of reactions included. A steady or slightlyfalling curve in Figure 24 generally suggests domination by a capture reaction, whereas a sharply-rising curve suggests a threshold reaction. For example, the calculation of ${ }^{45} \mathrm{Ca}$ production includes two reactions, ${ }^{44} \mathrm{C}(\mathrm{n}, \gamma)$ and ${ }^{48} \mathrm{Ti}(\mathrm{n}, \mathrm{a})$. The former is a capture reaction with a cross-section which decreases monotonically with increasing neutron energy above $1 \mathrm{MeV}$. The latter reaction has a threshold above $6 \mathrm{MeV}$, corresponding to the rise in ${ }^{45} \mathrm{Ca}$ production in granite for incident neutron energies above $6 \mathrm{MeV}$. 


\begin{abstract}
Summary
Using a simplified model for the interaction of energetic neutrons with granite and the MCNP code, we find that the energy-dependence of energy deposition by neutrons in granite follows from a balance of competing nuclear reactions primarily involving ${ }^{16} \mathrm{O}$ and ${ }^{28} \mathrm{Si}$ targets. Incorporation of water in the granite enhances energy deposition. The strong dependence of the quantities of certain radionuclides generated by neutron irradiation on the energy of the incident neutrons follows from the energy-dependence of the cross-sections of the various reaction channels which contribute to production of each radionuclide.
\end{abstract}

\title{
Acknowledgments
}

The authors are grateful to T. Scott Carman, D. Dearborn, T. Hoover, and P. Stry, and T. Thomson for helpful discussions, to D. McNabb and M. McKinley for assistance with access to nuclear data, and to L. Chase and R. Harding for assistance with the code. 


\section{Figures}

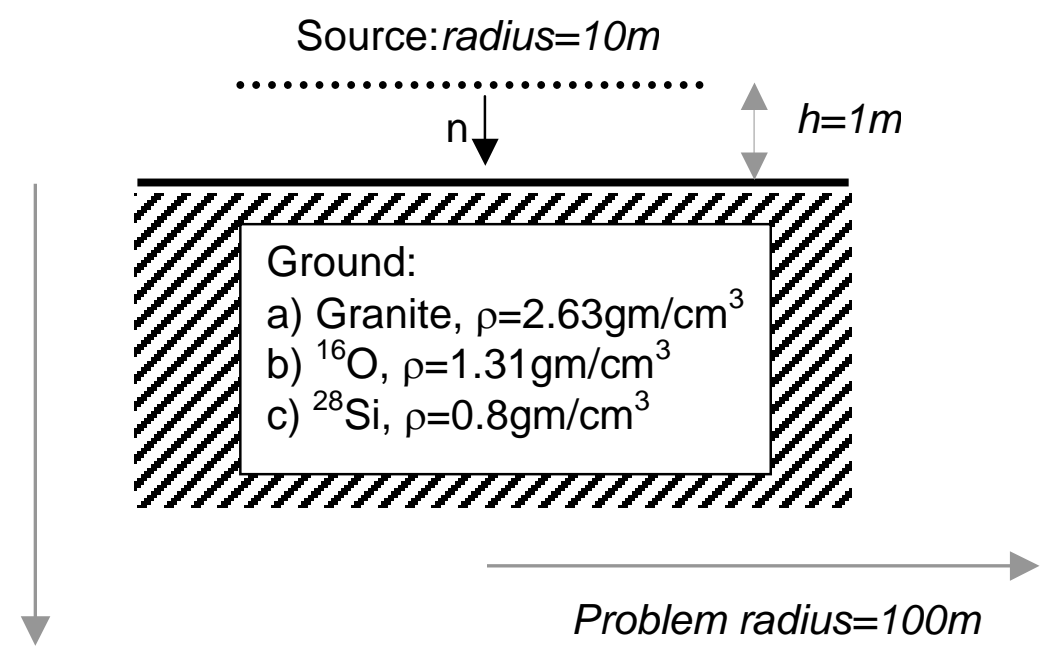

Problem depth $=32.6 \mathrm{~m}$

Figure 1: Test problem geometry. 
UCRL-TR-203529

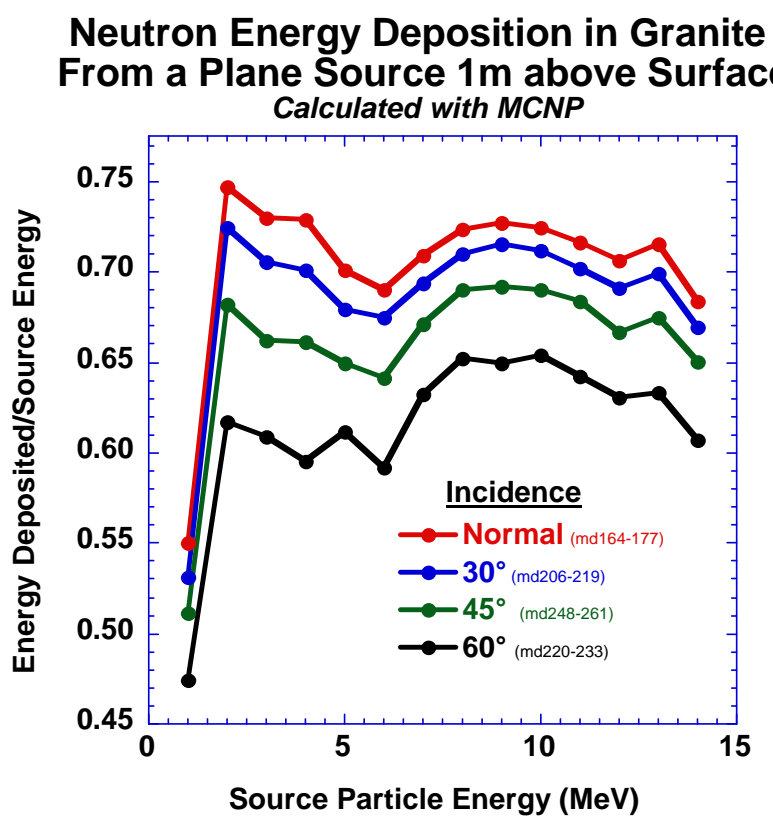

Figure 2: Calculated cumulative energy deposition into granite from monoenergetic, planar neutron sources with incident angles as indicated. Note zero suppression on the vertical scale.

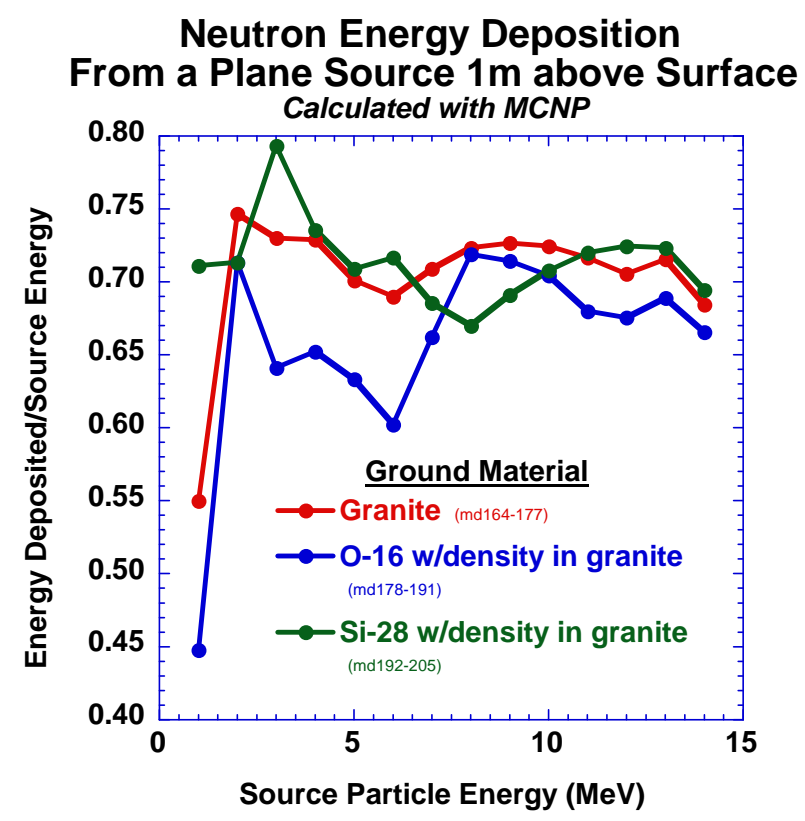

Figure 3: Calculated cumulative energy deposition into granite and into the principal atomic components of granite individually from monoenergetic, planar neutron sources and normal incidence. 
UCRL-TR-203529

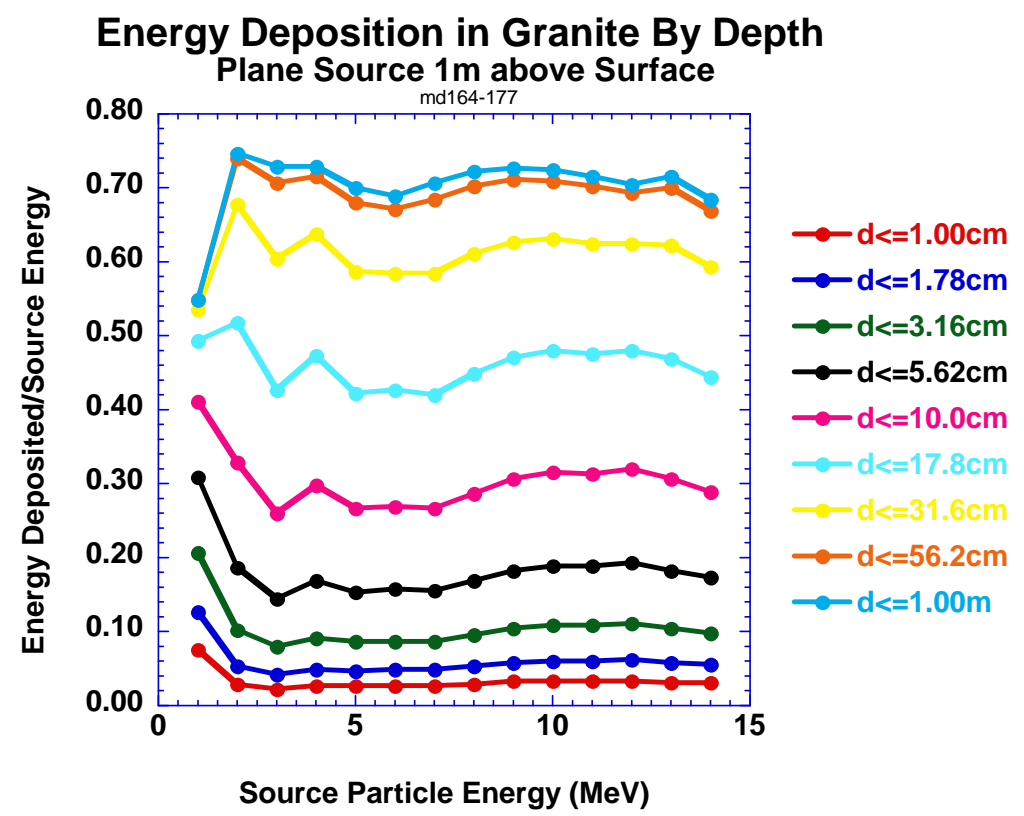

Figure 4: Calculated cumulative energy deposition into granite by depth. This and all subsequent plots involve neutrons at normal incidence.

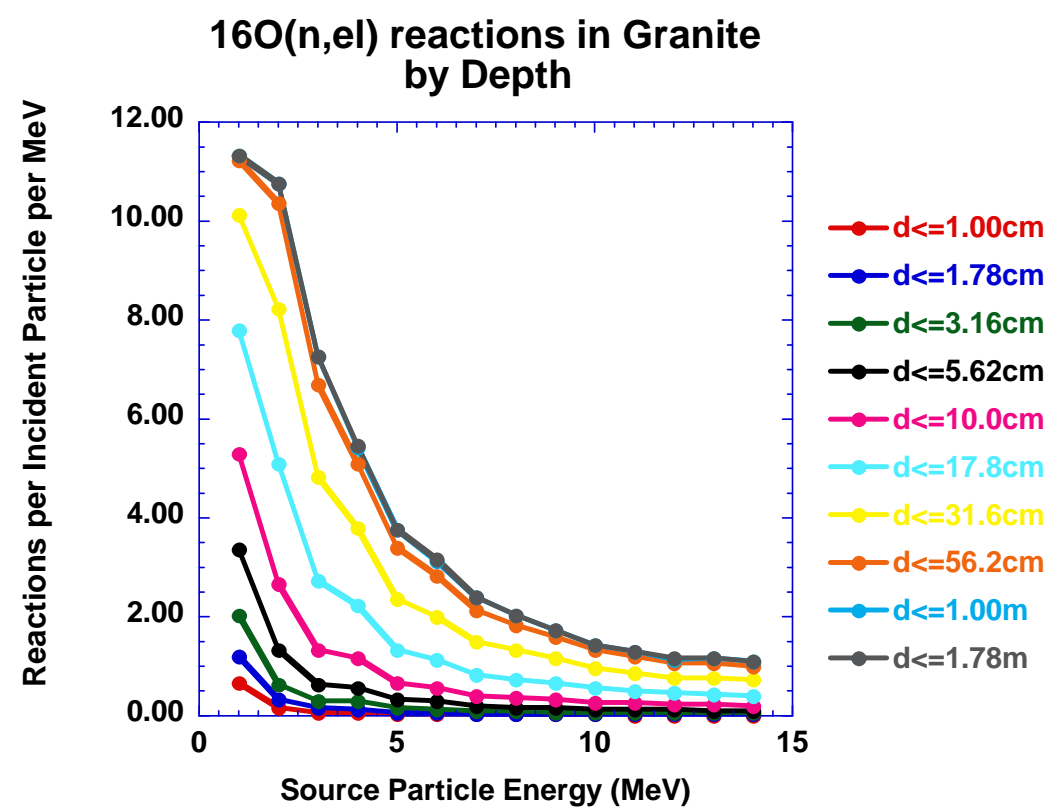

Figure 5: Calculated ${ }^{16} \mathrm{O}$ (n,elastic) reactions in granite by depth for normal incidence. 
UCRL-TR-203529

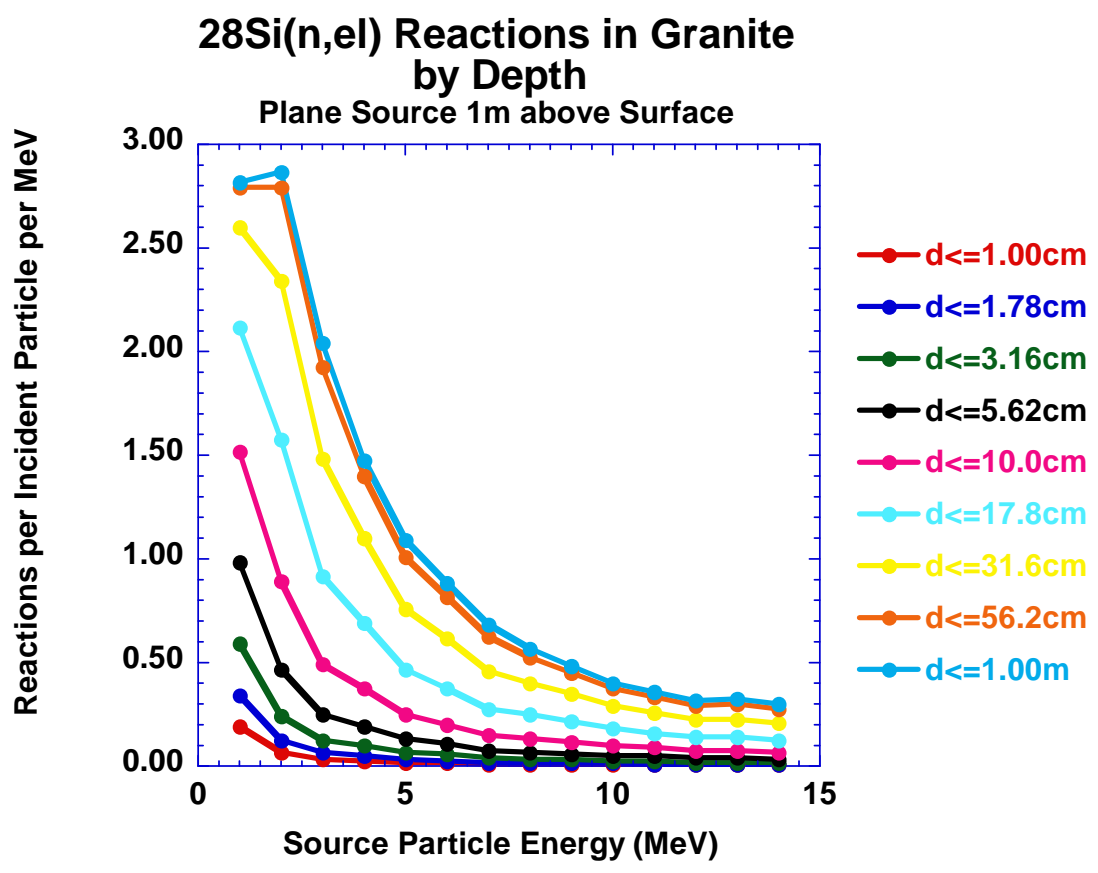

Figure 6: Calculated ${ }^{28} \mathrm{Si}(\mathrm{n}$,elastic) reactions in granite by depth.

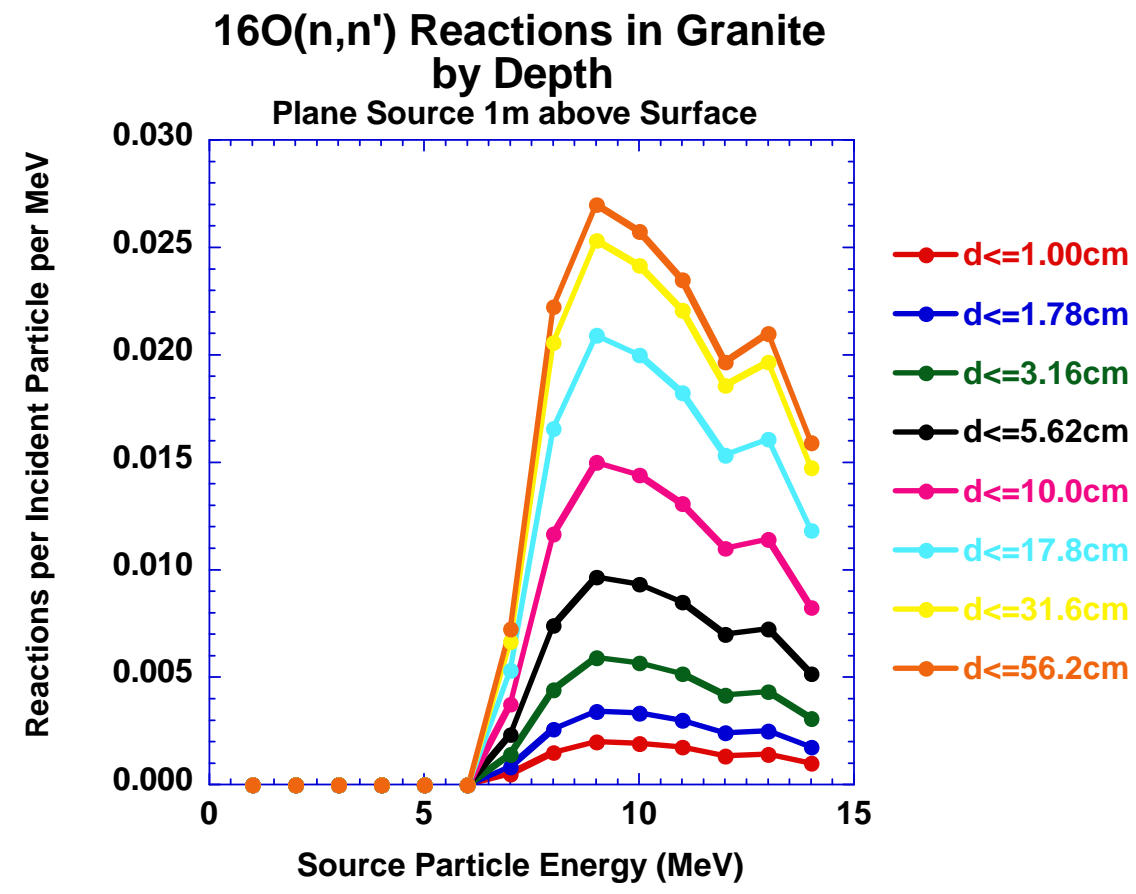

Figure 7: Calculated inelastic reactions of neutrons and ${ }^{16} \mathrm{O}$ in granite by depth. 
UCRL-TR-203529

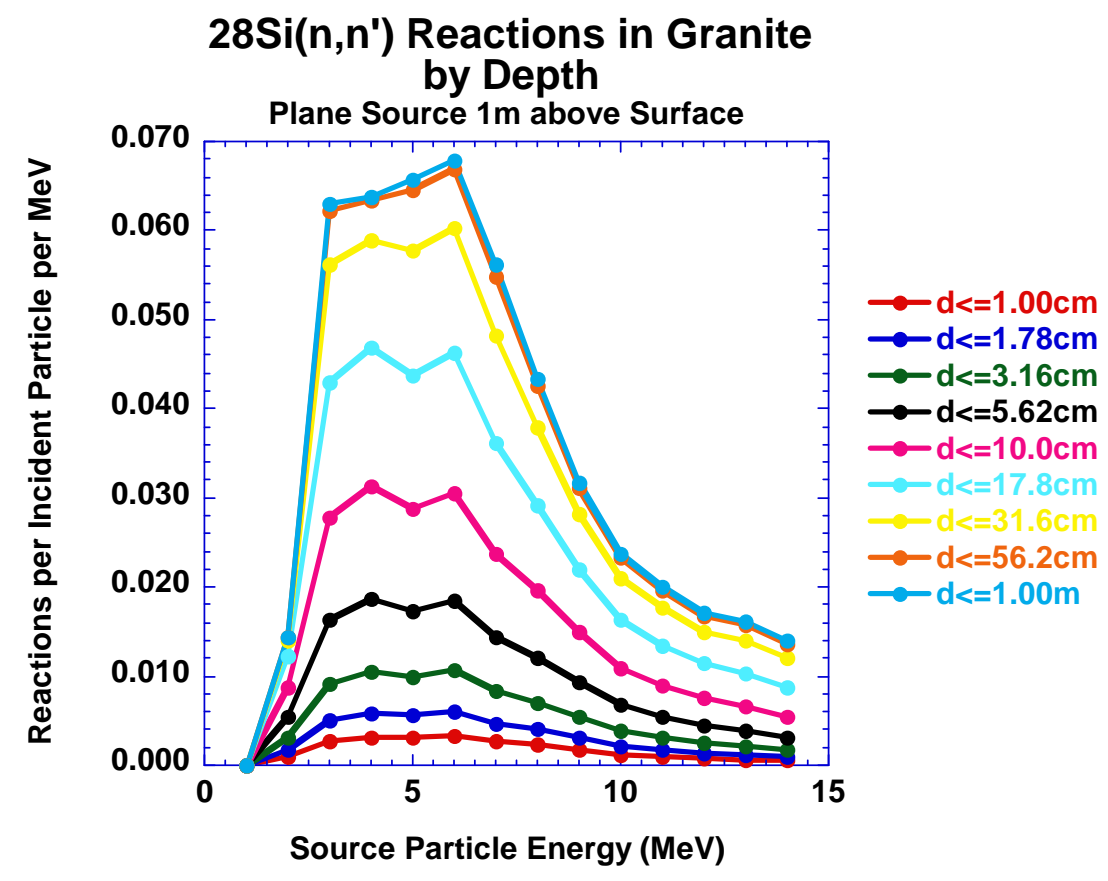

Figure 8: Calculated inelastic reactions of neutrons and ${ }^{28} \mathrm{Si}$ in granite by depth.

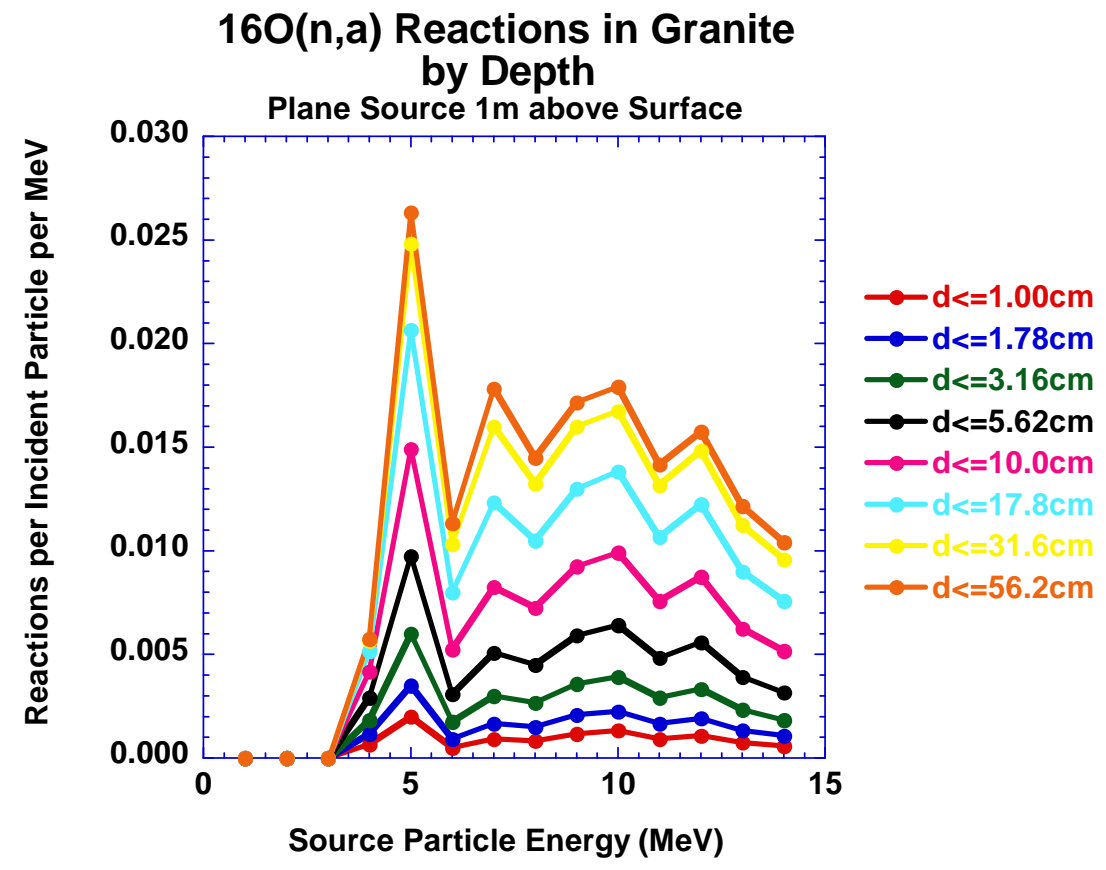

Figure 9: Calculated ${ }^{16} \mathrm{O}(\mathrm{n}, \alpha)$ reactions in granite by depth. 
UCRL-TR-203529

160(n,p) Reactions in Granite by Depth

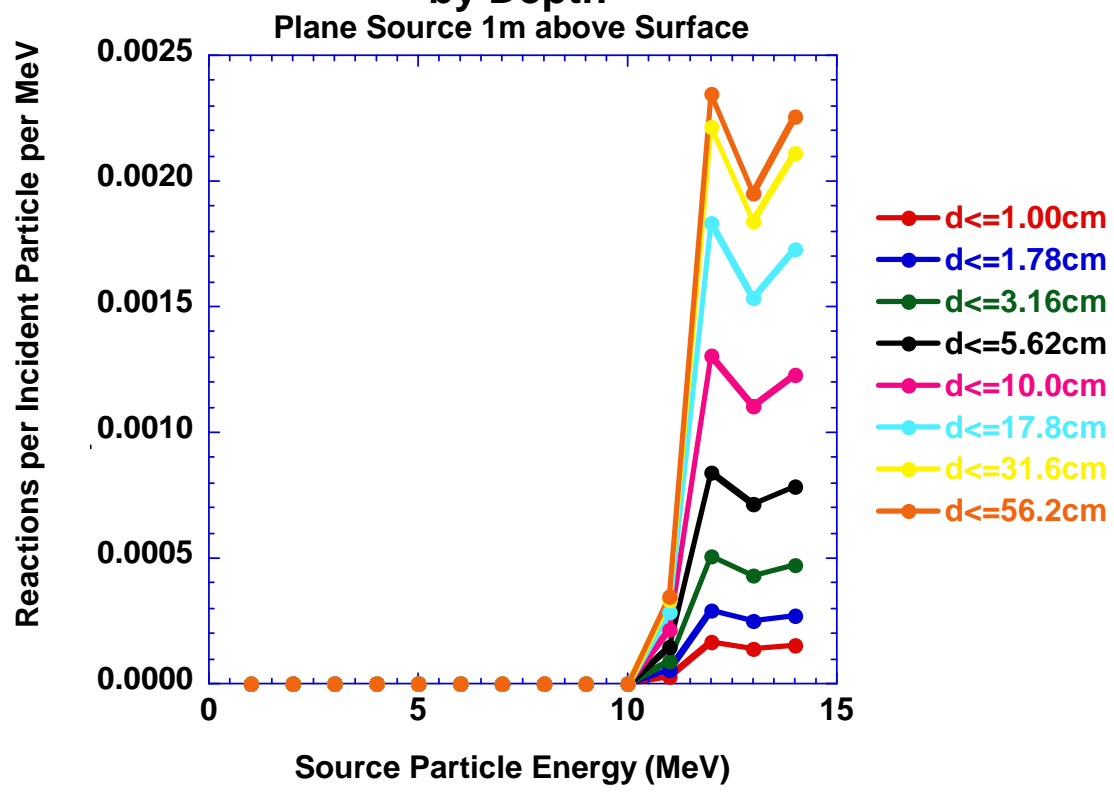

Figure 10: Calculated ${ }^{16} \mathrm{O}(\mathrm{n}, \mathrm{p})$ reactions in granite by depth.

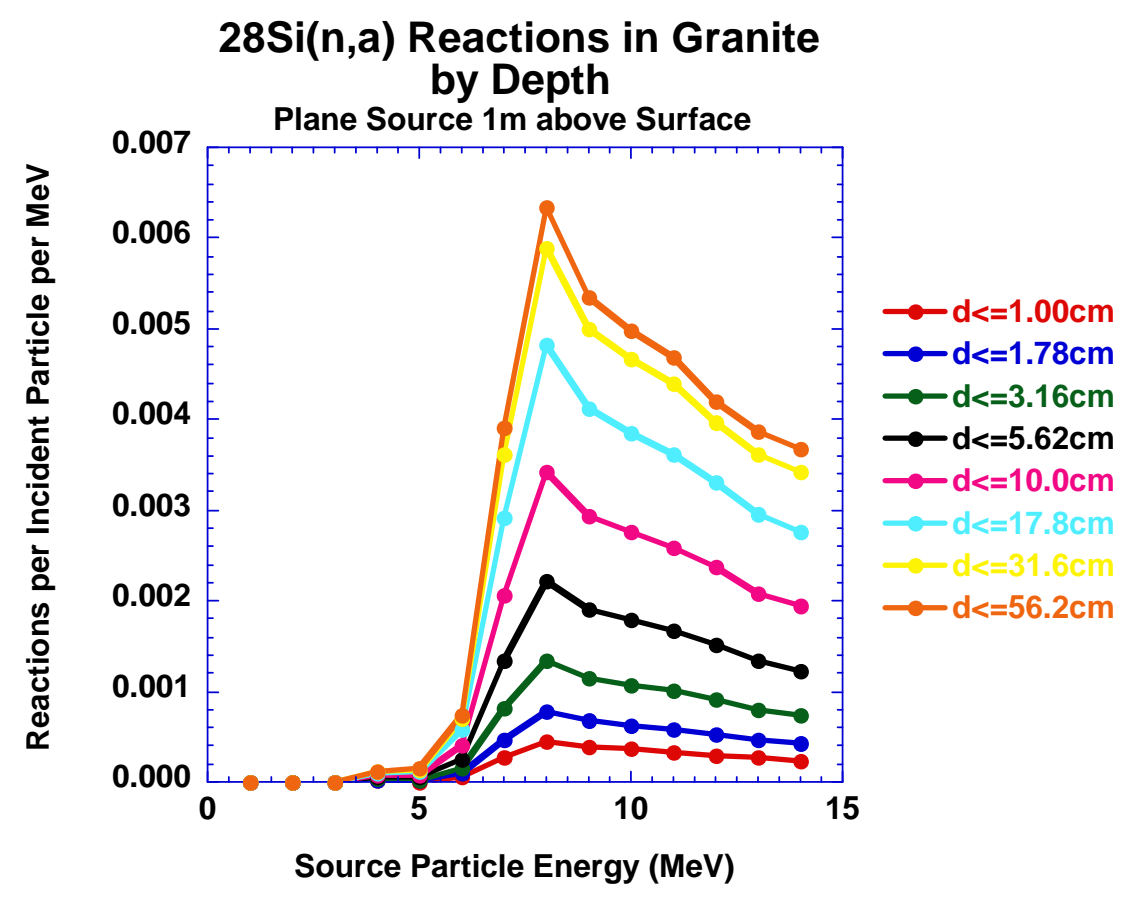

Figure 11: Calculated ${ }^{28} \mathrm{Si}(\mathrm{n}, \alpha)$ reactions in granite by depth. 
UCRL-TR-203529

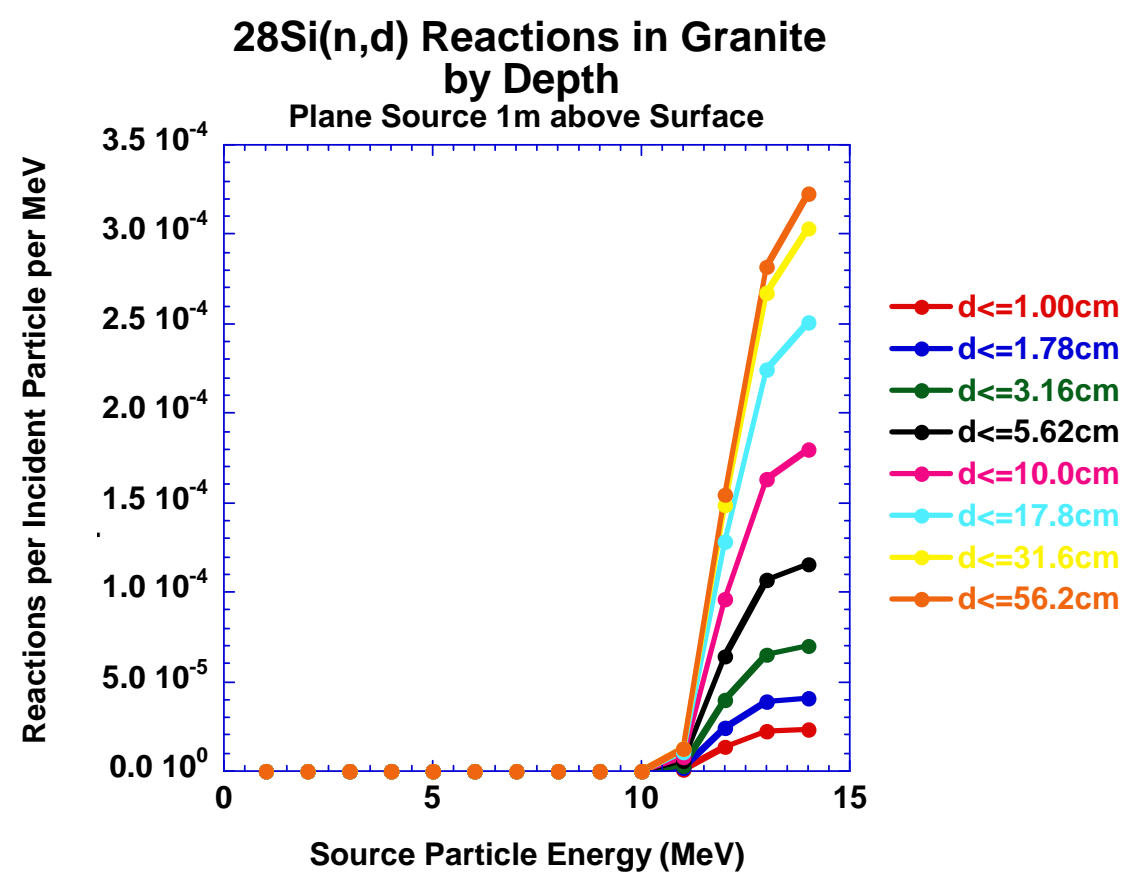

Figure 12: Calculated ${ }^{28} \mathrm{Si}(\mathrm{n}, \mathrm{d})$ reactions in granite by depth.

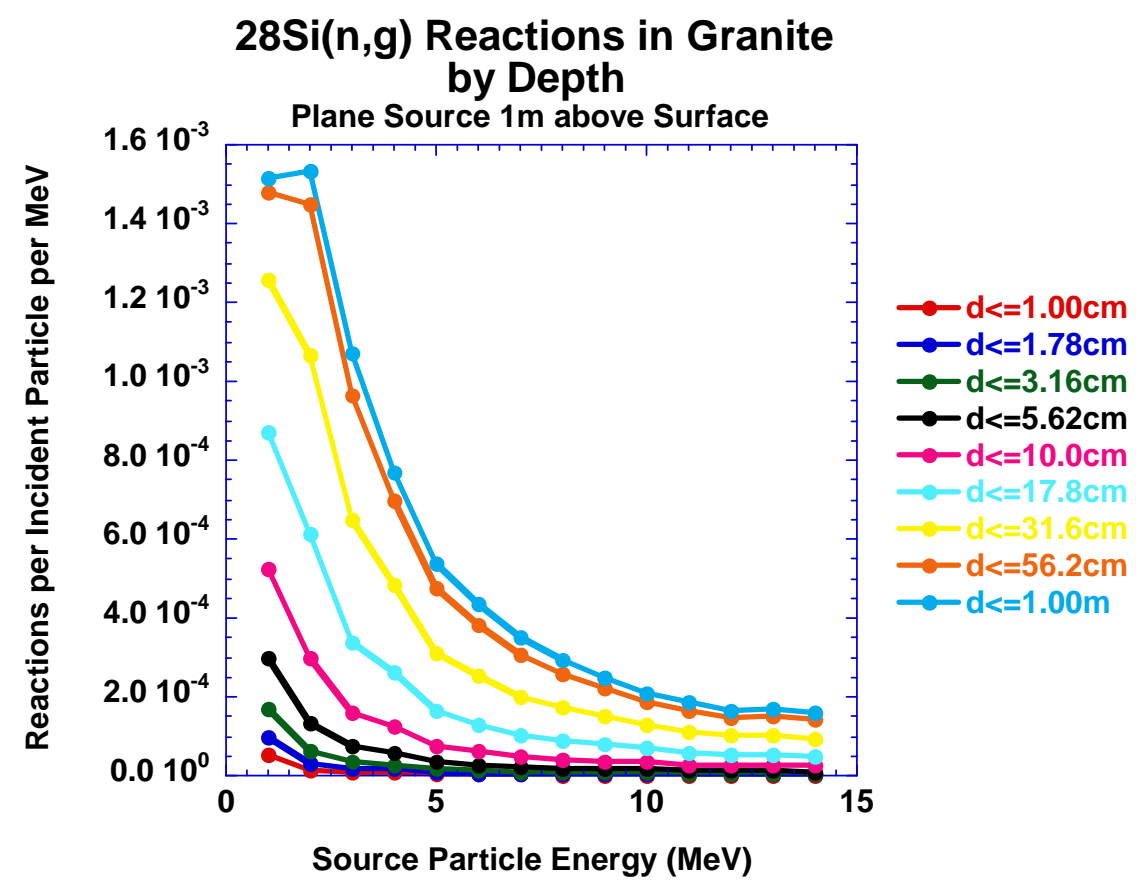

Figure 13: Calculated ${ }^{28} \mathrm{Si}(\mathrm{n}, \gamma)$ reactions in granite by depth. 
UCRL-TR-203529

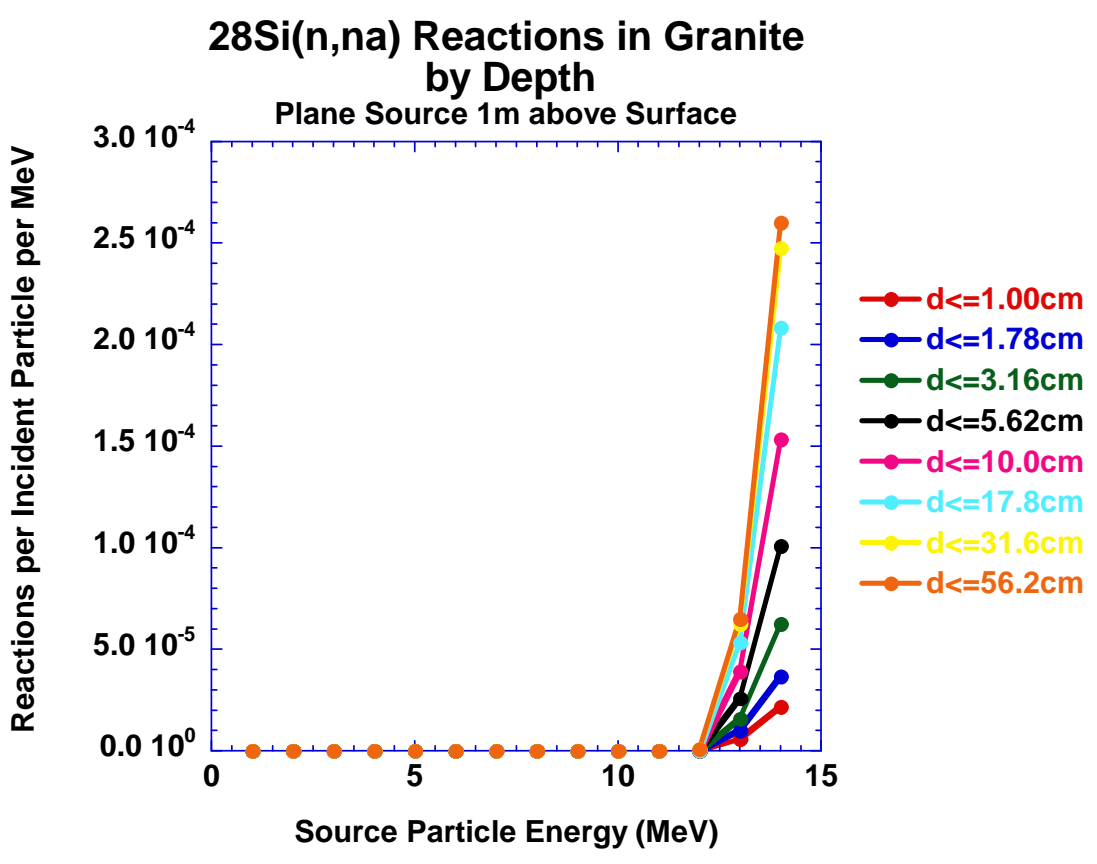

Figure 14: Calculated ${ }^{28} \mathrm{Si}(\mathrm{n}, \mathrm{n} \alpha)$ reactions in granite by depth.

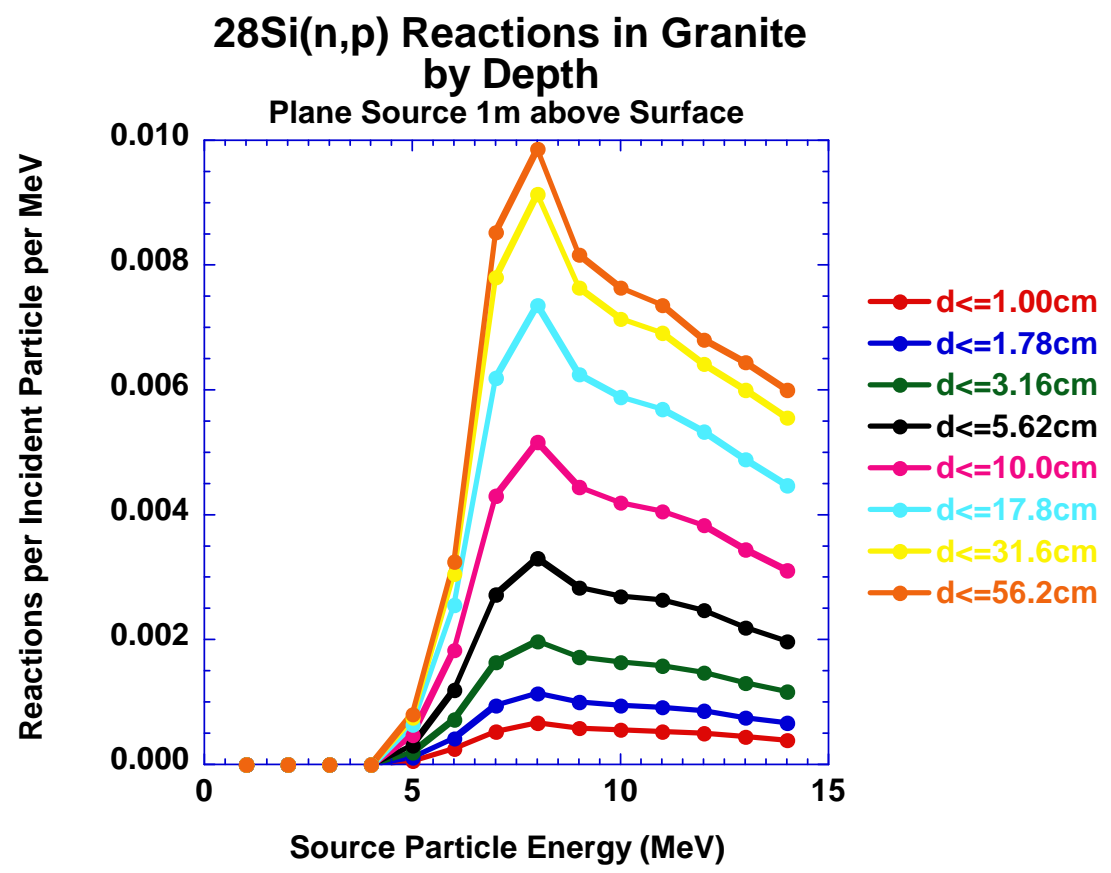

Figure 15: Calculated ${ }^{28} \mathrm{Si}(\mathrm{n}, \mathrm{p})$ reactions in granite by depth. 
UCRL-TR-203529

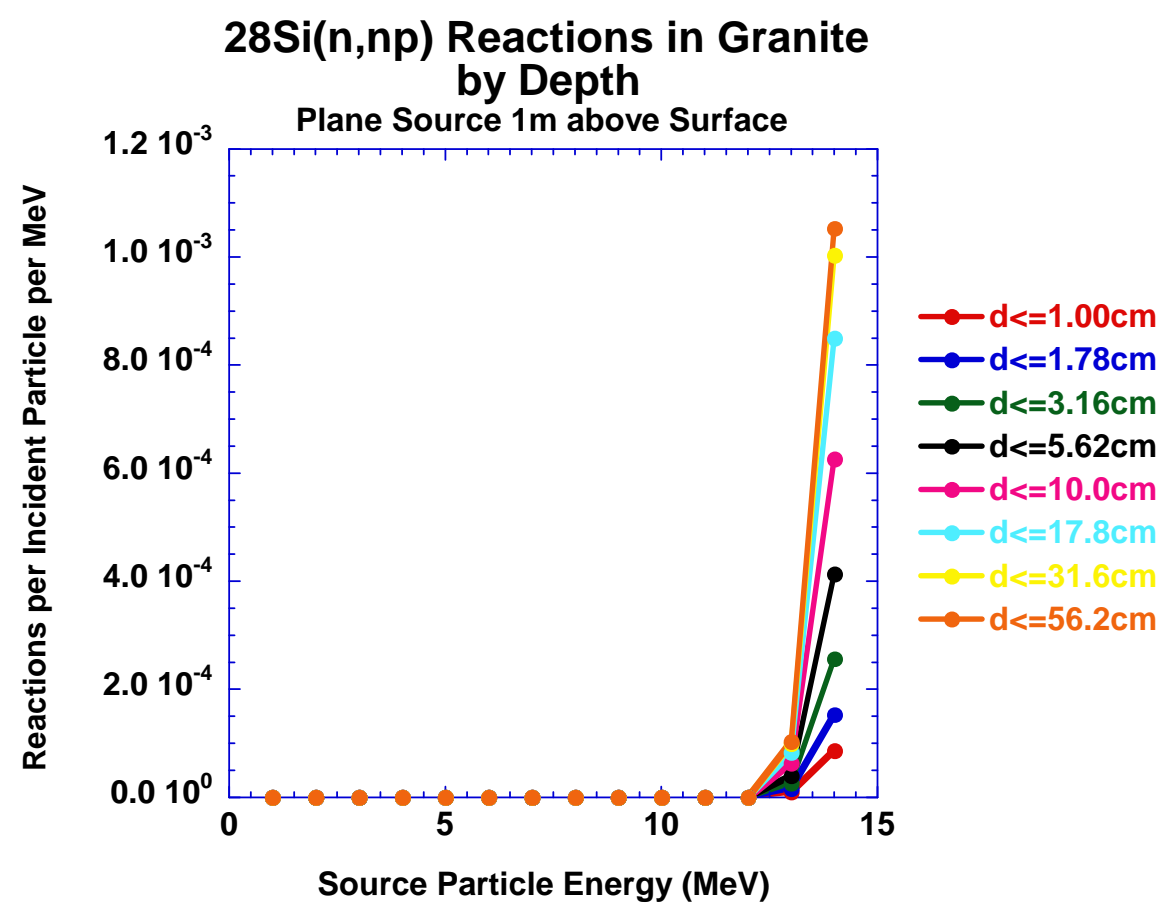

Figure 16: Calculated ${ }^{28} \mathrm{Si}(\mathrm{n}, \mathrm{np})$ reactions in granite by depth. 
UCRL-TR-203529

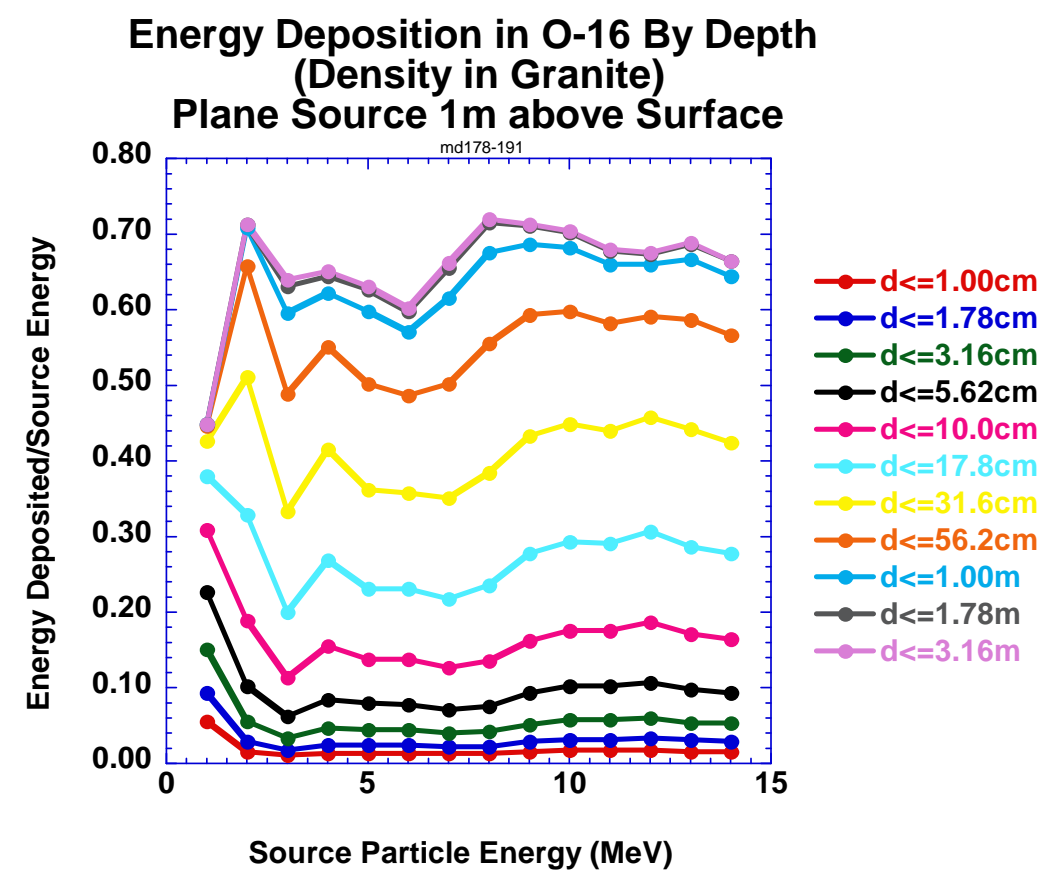

Figure 17: Calculated energy deposition by depth into the ${ }^{16} \mathrm{O}$ component of granite only. Other components are removed from the problem.

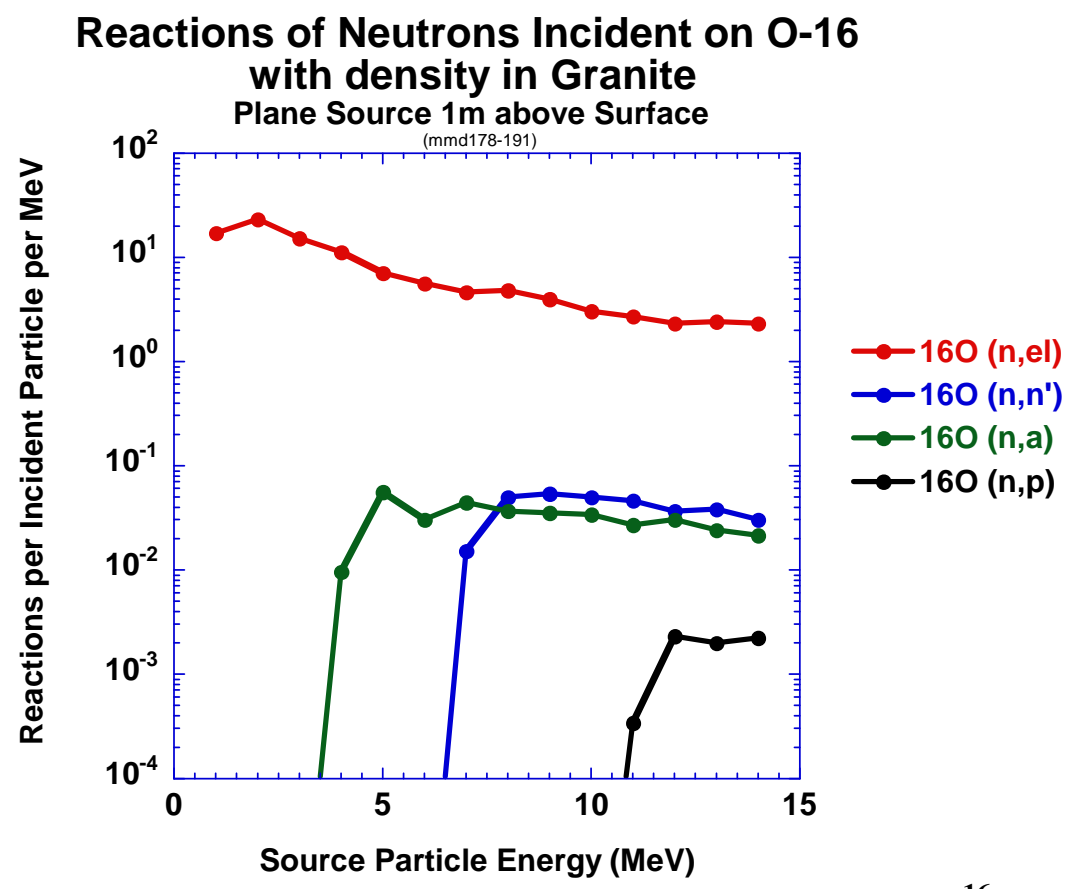

Figure 18: Calculated, total neutron reactions by depth into the ${ }^{16} \mathrm{O}$ component of granite only. Other components are removed from the problem. 
UCRL-TR-203529

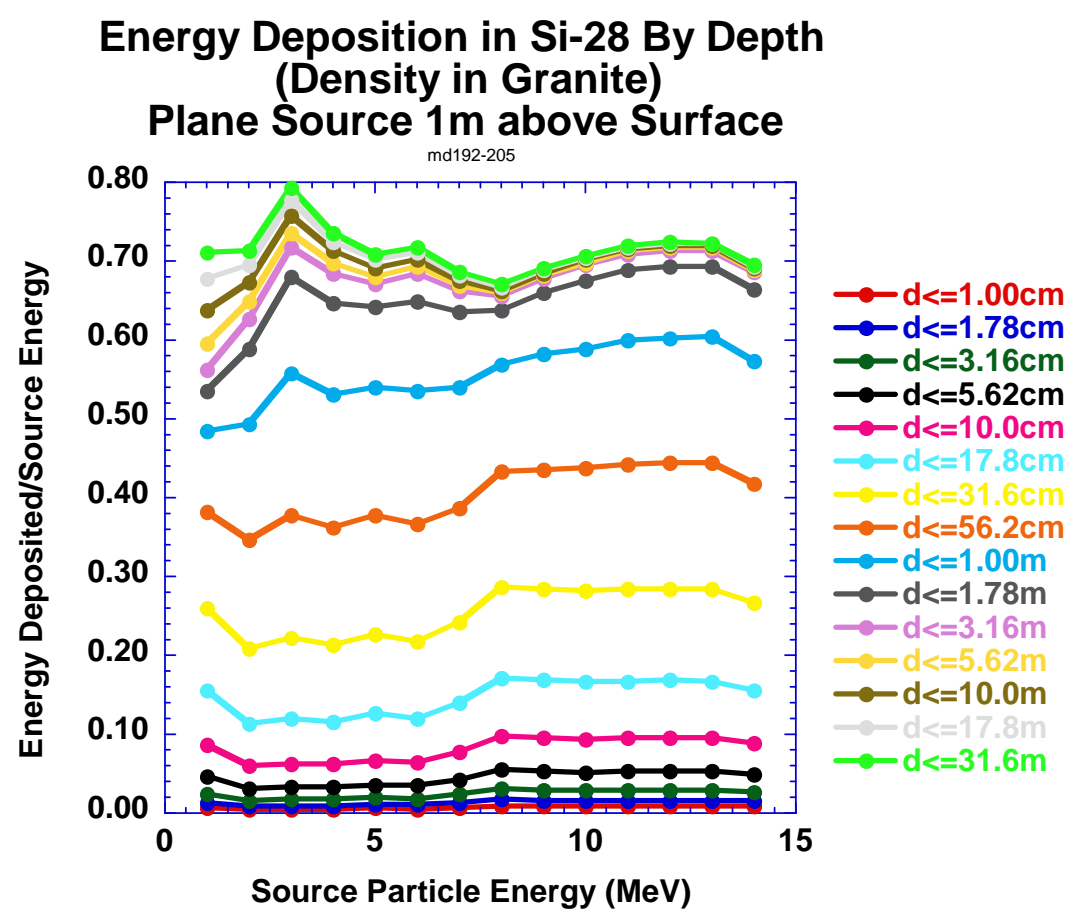

Figure 19: Calculated energy deposition by depth into the ${ }^{28} \mathrm{Si}$ component of granite only. Other components are removed from the problem.

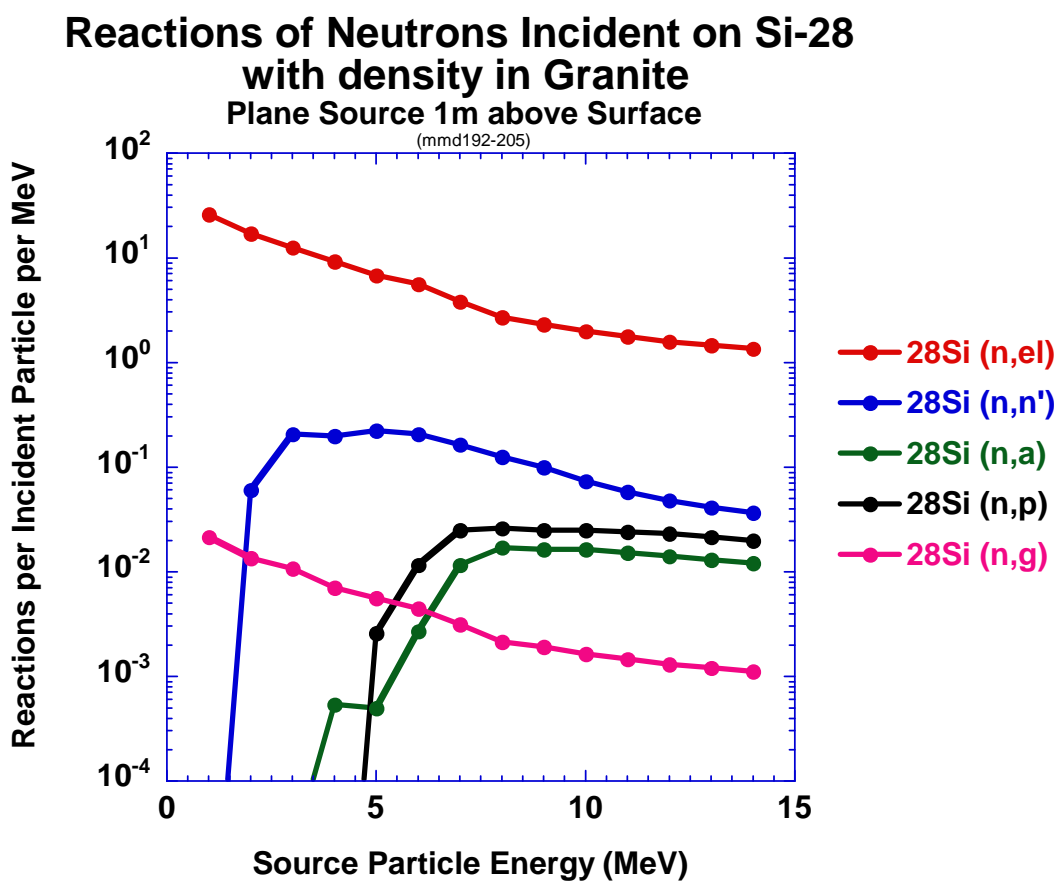

Figure 20: Calculated, total neutron reactions by depth into the ${ }^{28} \mathrm{Si}$ component of granite only. Other components are removed from the problem. 
UCRL-TR-203529

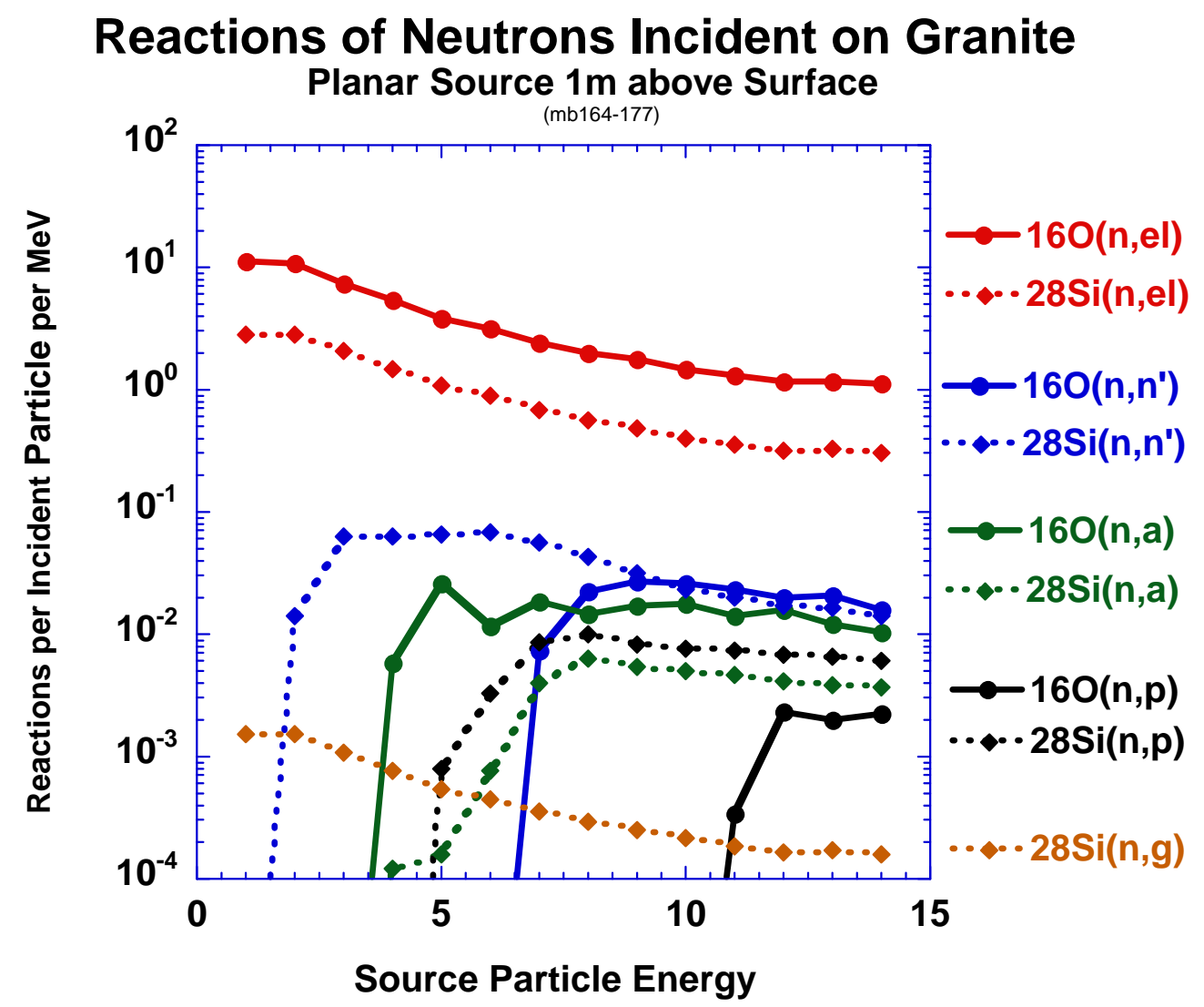

Figure 21: Summary of the dominant reactions for neutrons incident on granite. Reactions involving ${ }^{16} \mathrm{O}$ are represented with solid curves, while reactions involving ${ }^{28} \mathrm{Si}$ are represented with dashed curves. 
UCRL-TR-203529

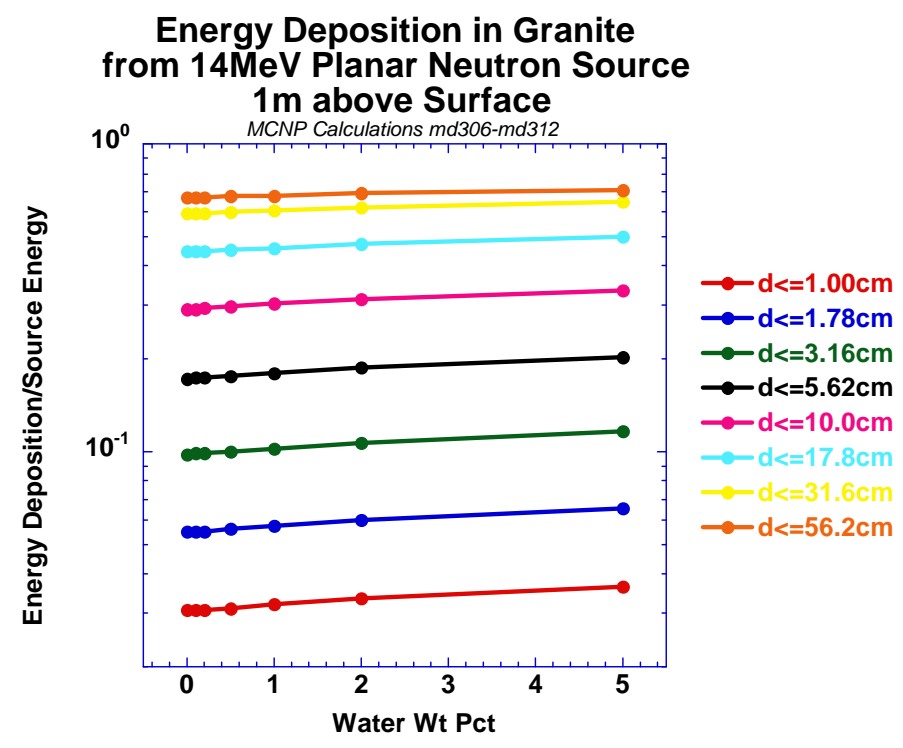

Figure 22: Calculated energy deposition by depth in granite vs. percentage of added water.
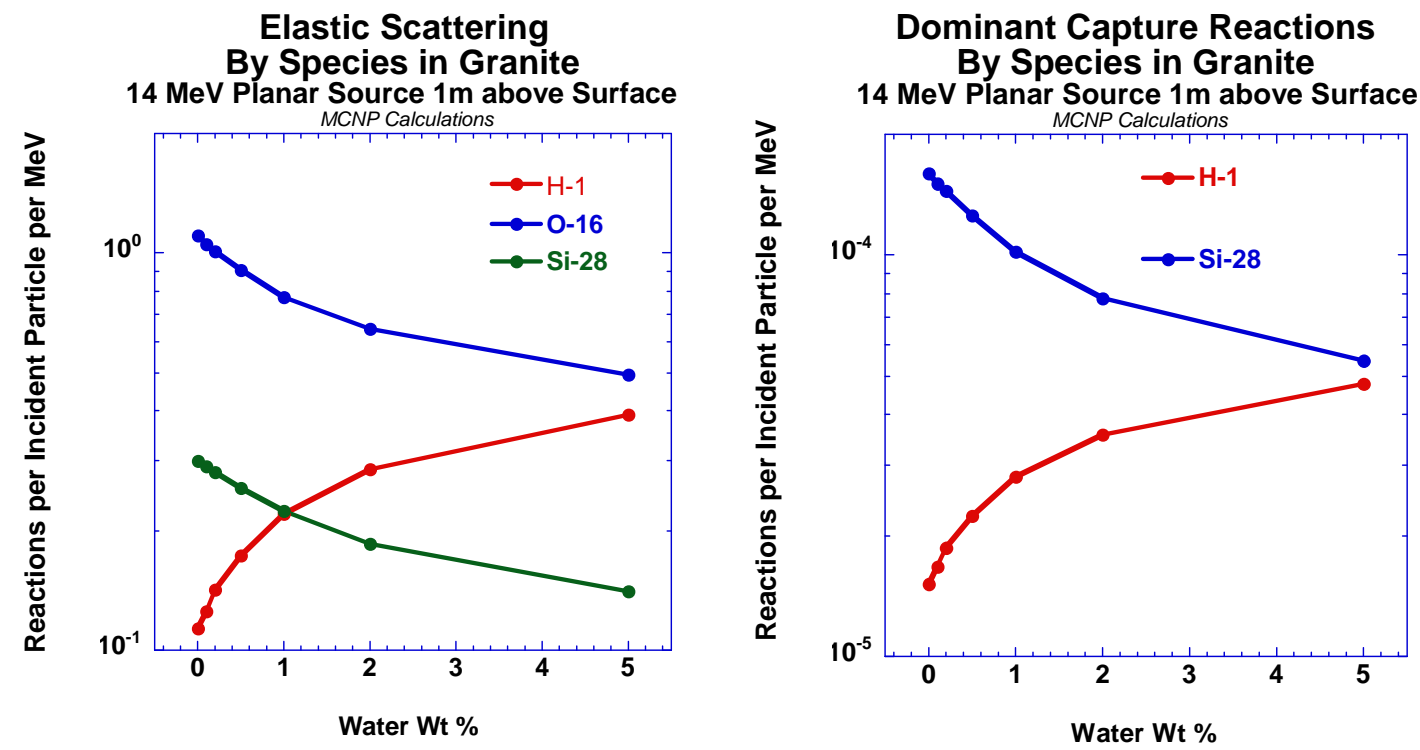

Figure 23: Effects of added water on neutron reactions in granite. 
UCRL-TR-203529
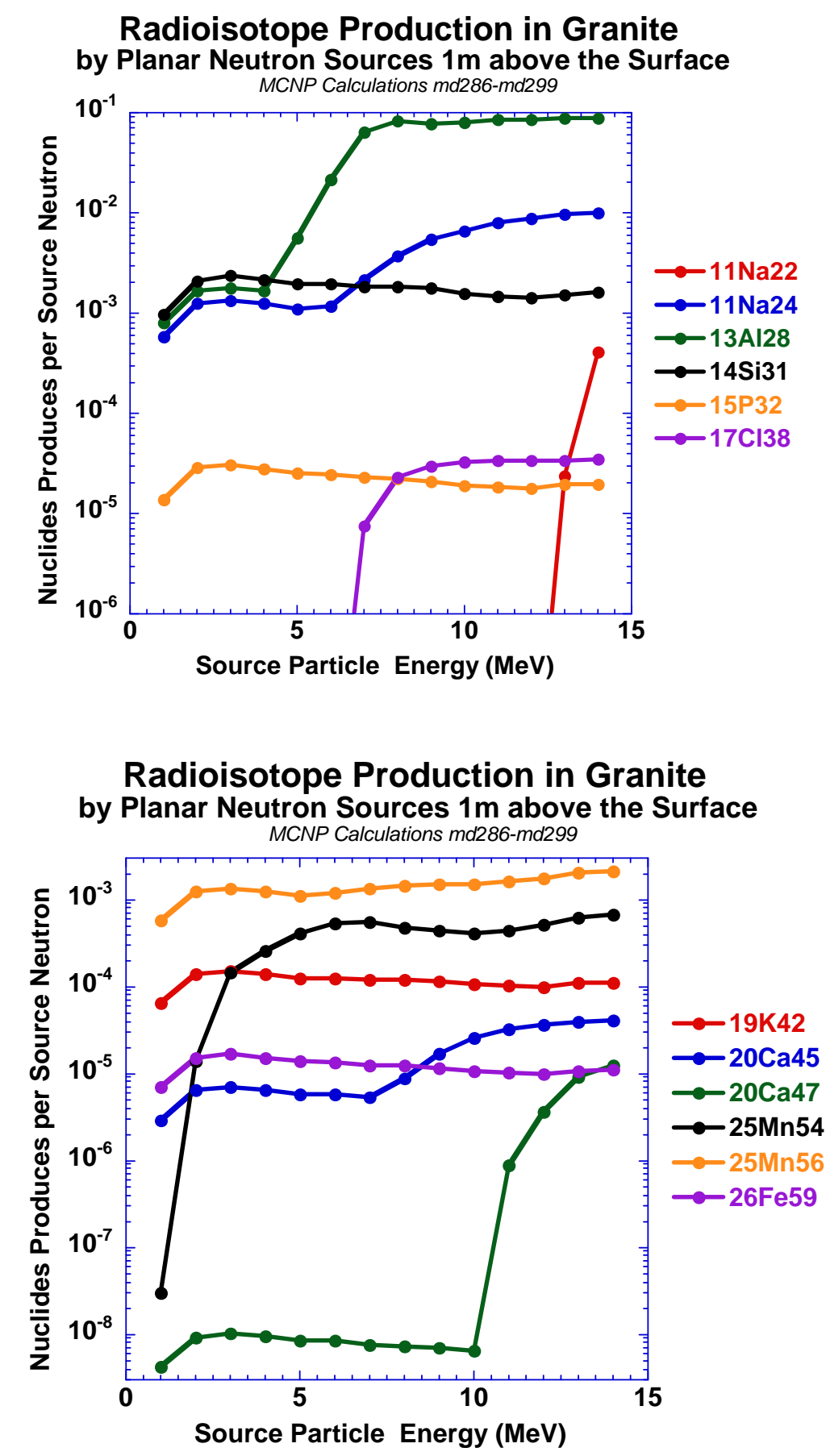

Figure 24: Total production of specific radioisotopes in granite from planar, monoenergetic neutron sources. 
UCRL-TR-203529

Tables

Simplified granite composition.

\begin{tabular}{|c|c|c|c|}
\hline Species & $\underline{\text { Wt Fraction }}$ & $\begin{array}{l}\text { Wt Fraction in } \\
\text { Earth's Crust }^{11} \\
\end{array}$ & $\begin{array}{l}\text { Wt Fraction } \\
\text { of pure } \mathrm{SiO}\end{array}$ \\
\hline H & 0.00093 & 0.0014 & \\
\hline 0 & 0.49813 & 0.461 & 0.533 \\
\hline $\mathrm{Na}$ & 0.02582 & 0.0236 & \\
\hline Mg & 0.00528 & 0.0233 & \\
\hline Al & 0.07661 & 0.0823 & \\
\hline Si & 0.32751 & 0.282 & 0.467 \\
\hline $\mathbf{P}$ & 0.00083 & 0.00105 & \\
\hline K & 0.02258 & 0.0209 & \\
\hline $\mathrm{Ca}$ & 0.01421 & 0.0415 & \\
\hline $\mathrm{Ti}$ & 0.00234 & 0.00565 & \\
\hline Mn & 0.00093 & 0.000095 & \\
\hline $\mathrm{Fe}$ & 0.02483 & 0.0563 & \\
\hline
\end{tabular}

Table 1: Composition of simplified granite used in this study. The calculations reflect the natural isotopic abundances for each element.

${ }^{11}$ Source: CRC Handbook of Chemistry and Physics (Boca Raton: CRC Press, 1998), p. 14-14. 
UCRL-TR-203529

Residual Radionuclides and their decay properties.

\begin{tabular}{|c|c|c|c|c|c|c|c|}
\hline \multicolumn{2}{|c|}{ Radionuclide } & \multirow{2}{*}{$\frac{\text { Half-Life }}{5730 y}$} & Decay & Prod. & \multirow[t]{2}{*}{ Half-Life } & \multirow[t]{2}{*}{ Decay } & Prod \\
\hline $6 \mathrm{C}$ & 14 & & $7 \mathrm{~N}$ & 14 & & & \\
\hline $11 \mathrm{Na}$ & 22 & $2.62 y$ & $10 \mathrm{Na}$ & 22 & & & \\
\hline $11 \mathrm{Na}$ & 24 & $15 \mathrm{~h}$ & $12 \mathrm{Mg}$ & 24 & & & \\
\hline $13 \mathrm{Al}$ & 28 & $2.3 \mathrm{~m}$ & $14 \mathrm{Si}$ & 28 & & & \\
\hline $14 \mathrm{Si}$ & 31 & $2.62 \mathrm{~h}$ & $15 \mathrm{P}$ & 31 & & & \\
\hline $14 \mathrm{P}$ & 32 & $24 d$ & $16 \mathrm{~S}$ & 32 & & & \\
\hline $17 \mathrm{Cl}$ & 38 & $37.3 \mathrm{~m}$ & $18 \mathrm{Ar}$ & 38 & & & \\
\hline $19 \mathrm{~K}$ & 42 & $12.36 \mathrm{~h}$ & $20 \mathrm{Ca}$ & 42 & & & \\
\hline $20 \mathrm{Ca}$ & 45 & $165 d$ & $21 \mathrm{Sc}$ & 45 & & & \\
\hline $20 \mathrm{Ca}$ & 47 & $4.535 d$ & $21 \mathrm{Sc}$ & 47 & $3.44 \mathrm{~d}$ & $22 \mathrm{Ti}$ & 47 \\
\hline $25 \mathrm{Mn}$ & 54 & $303 d$ & $24 \mathrm{Cr}$ & 54 & & & \\
\hline $25 \mathrm{Mn}$ & 56 & $2.576 h$ & $26 \mathrm{Fe}$ & 56 & & & \\
\hline $26 \mathrm{Fe}$ & 59 & $44.6 d$ & $27 \mathrm{Co}$ & 59 & & & \\
\hline $27 \mathrm{Co}$ & 60 & $5.26 y$ & $28 \mathrm{Ni}$ & 60 & & & \\
\hline $28 \mathrm{Ni}$ & 65 & $2.56 h$ & $29 \mathrm{Cu}$ & 65 & & & \\
\hline $29 \mathrm{Cu}$ & 64 & $12.8 \mathrm{~h}$ & $30 \mathrm{zn}$ & 64 & (or 28Ni64) & & \\
\hline $30 \mathrm{zn}$ & 65 & $245 d$ & $29 \mathrm{Cu}$ & 65 & & & \\
\hline $38 \mathrm{Sr}$ & 89 & $52 d$ & $39 Y$ & 89 & & & \\
\hline $39 \mathrm{Sr}$ & 91 & $9.7 \mathrm{~h}$ & $39 Y$ & 91 & $58 d$ & $40 \mathrm{zr}$ & 91 \\
\hline
\end{tabular}

Table 2: Radionuclides included in published lists of contaminant nuclides which may be created by neutron irradiation of ground. Those nuclides shown in red are radioactive. Note that not all of these radionuclides can be produced from the isotopes included in the simplified granite of Table 1, but they may be produced by neutron reactions with trace elements. 
Channels with available cross-sections for producing residual radionuclides.

\begin{tabular}{|c|c|c|c|c|c|c|c|}
\hline Product & $\underline{\text { Library }}$ & $\underline{\text { Target }}$ & $\underline{\mathrm{Rxn}}$ & Product & $\underline{\text { Library }}$ & Target & $\underline{\mathrm{R} \times \mathrm{n}}$ \\
\hline$C-14$ & ENDFb 6 & $\mathrm{~N}-15$ & $(n, n p)$ & $\mathrm{Ca}-47$ & ACTL & $\mathrm{Ca}-48$ & $(n, 2 n)$ \\
\hline$C-14$ & ENDL 92 & $C-13$ & $(n, g)$ & $\mathrm{Ca}-47$ & ACTL & $\mathrm{Ca}-46$ & $(n, g)$ \\
\hline$C-14$ & ENDFb 6 & $\mathrm{~N}-14$ & $(n, p)$ & $\mathrm{Ca}-47$ & ENDFb 6 & $\mathrm{Ti}-50$ & $(n, a)$ \\
\hline$C-14$ & ENDFb 6 & $\mathrm{~N}-15$ & $(n, d)$ & $M n-54$ & ENDFb 6 & $M n-55$ & $(n, 2 n)$ \\
\hline$C-14$ & ENDFb 6 & $0-17$ & $(n, a)$ & $M n-54$ & ENDFb 6 & $\mathrm{Fe}-54$ & $(n, p)$ \\
\hline $\mathrm{Na}-22$ & ENDFb 6 & $\mathrm{Na}-23$ & $(n, 2 n)$ & $M n-54$ & ENDFb 6 & $\mathrm{Fe}-56$ & $(n, t)$ \\
\hline $\mathrm{Na}-24$ & ENDFb 6 & $\mathrm{Na}-23$ & $(n, g)$ & $M n-56$ & ENDFb 6 & $\mathrm{Fe}-57$ & $(n, n p)$ \\
\hline $\mathrm{Na}-24$ & ENDFb 6 & $M g-24$ & $(\mathrm{n}, \mathrm{p})$ & $M n-56$ & ENDFb 6 & $M n-55$ & $(n, g)$ \\
\hline $\mathrm{Na}-24$ & ENDFb 6 & $\mathrm{Al}-27$ & $(n, a)$ & $M n-56$ & ENDFb 6 & $\mathrm{Fe}-56$ & $(n, p)$ \\
\hline$A l-28$ & ENDFb 6 & Si-29 & $(n, n p)$ & $M n-56$ & ACTL & $\mathrm{Fe}-58$ & $(n, t)$ \\
\hline Al-28 & ENDFb 6 & $\mathrm{~A} I-27$ & $(n, g)$ & $\mathrm{Mn}-56$ & ENDFb 6 & Co-59 & $(n, a)$ \\
\hline Al-28 & ENDFb 6 & $\mathrm{Si}-28$ & $(n, p)$ & $F e-59$ & ENDFb 6 & $\mathrm{Fe}-58$ & $(n, g)$ \\
\hline $\mathrm{Al}-28$ & ENDFb 6 & $P-31$ & $(n, a)$ & $F e-59$ & ENDFb 6 & Co-59 & $(n, p)$ \\
\hline $\mathrm{Si}-31$ & ENDFb 6 & $\mathrm{Si}-30$ & $(n, g)$ & $\mathrm{Fe}-59$ & ENDFb 6 & $\mathrm{Ni}-62$ & $(n, a)$ \\
\hline Si-31 & ENDFb 6 & $P-31$ & $(n, p)$ & $\mathrm{Co}-60$ & ENDFb 6 & $\mathrm{Ni}-61$ & $(n, n p)$ \\
\hline Si-31 & ACTL & $s-34$ & $(n, a)$ & Co- 60 & ENDFb 6 & Co-59 & $(n, g)$ \\
\hline$P-32$ & ACTL & $s-33$ & $(n, n p)$ & $\mathrm{Co}-60$ & ENDFb 6 & $\mathrm{Ni}-60$ & $(\mathrm{n}, \mathrm{p})$ \\
\hline$P-32$ & ENDFb 6 & $P-31$ & $(n, g)$ & $\mathrm{Co}-60$ & ACTL & $\mathrm{Ni}-61$ & $(n, d)$ \\
\hline$P-32$ & ENDFb 6 & $s-32$ & $(n, p)$ & $\mathrm{Co}-60$ & ACTL & $\mathrm{Ni}-62$ & $(n, t)$ \\
\hline$P-32$ & ACTL & $s-33$ & $(n, d)$ & $\mathrm{Co}-60$ & ENDFb 6 & $\mathrm{Cu}-63$ & $(n, a)$ \\
\hline$P-32$ & ACTL & $\mathrm{Cl}-35$ & $(n, a)$ & $\mathrm{Ni}-65$ & ENDFb 6 & $\mathrm{Ni}-64$ & $(n, g)$ \\
\hline $\mathrm{Cl}-38$ & ACTL & $\mathrm{Cl}-37$ & $(n, g)$ & $\mathrm{Ni}-65$ & ENDFb 6 & $\mathrm{Cu}-65$ & $(n, p)$ \\
\hline $\mathrm{Cl}-38$ & ACTL & Ar-38 & $(n, p)$ & $\mathrm{Ni}-65$ & ACTL & $2 n-68$ & $(n, a)$ \\
\hline $\mathrm{Cl}-38$ & ACTL & $\mathrm{K}-41$ & $(n, a)$ & $\mathrm{Cu}-64$ & ENDFb 6 & $\mathrm{Cu}-65$ & $(n, 2 n)$ \\
\hline$K-42$ & ACTL & $K-41$ & $(n, g)$ & $\mathrm{Cu}-64$ & ENDFb 6 & $\mathrm{Cu}-63$ & $(n, g)$ \\
\hline $\mathrm{K}-42$ & ACTL & $\mathrm{Ca}-42$ & $(n, p)$ & $\mathrm{Cu}-64$ & ACTL & $\mathrm{Zn}-64$ & $(\mathrm{n}, \mathrm{p})$ \\
\hline$K-42$ & ACTL & $\mathrm{Ca}-43$ & $(n, d)$ & $Z n-65$ & ACTL & $Z n-66$ & $(n, 2 n)$ \\
\hline $\mathrm{K}-42$ & ENDFb 6 & $S C-45$ & $(\mathrm{n}, \mathrm{a})$ & $Z n-65$ & ACTL & $Z n-64$ & $(n, g)$ \\
\hline $\mathrm{Ca}-45$ & ACTL & $\mathrm{Ca}-46$ & $(n, 2 n)$ & Sr-89 & ENDFb 6 & $\mathrm{Sr}-88$ & $(n, g)$ \\
\hline $\mathrm{Ca}-45$ & ACTL & $\mathrm{Ti}-49$ & $(\mathrm{n}, \mathrm{na})$ & Sr-89 & ENDFb 6 & Zr-92 & $(n, a)$ \\
\hline $\mathrm{Ca}-45$ & ACTL & $\mathrm{Ca}-44$ & $(n, g)$ & Sr-90 & ACTL & Zr-94 & $(n, n a)$ \\
\hline $\mathrm{Ca}-45$ & ENDFb 6 & $S c-45$ & $(n, p)$ & Sr-91 & ENDFb 6 & Zr-94 & $(n, a)$ \\
\hline $\mathrm{Ca}-45$ & ENDFb 6 & $\mathrm{~T} i-48$ & $(n, a)$ & Sr-93 & ENDFb 6 & Zr-96 & $(n, a)$ \\
\hline
\end{tabular}

Table 3: Available cross-sections for producing each of the radionuclides listed in Table 2. The reactions included in calculations described in this work are highlighted in yellow. Those which are not highlighted involve target nuclides which are not included in the simplified granite formulation considered here. 
UCRL-TR-203529

\section{Residual Radionuclides with their parent chains and half-lives.}

Radionuclides produced bv decavs from parent chains. denotes production cross-section availabilitv.

\begin{tabular}{|c|c|c|c|c|c|c|c|c|c|}
\hline $\begin{array}{l}\text { Product } \\
\mathrm{C}-14\end{array}$ & $\begin{array}{l}\text { Half-Life } \\
5730 y\end{array}$ & $\begin{array}{l}\text { Parent } \\
\mid \begin{array}{l}\mathrm{B}-14 \\
\mathrm{~N}-18\end{array}\end{array}$ & $\begin{array}{l}\text { Half-Life } \\
13.8 \mathrm{~ms} \\
624 \mathrm{~ms}\end{array}$ & $\begin{array}{l}\text { Parent } \\
\begin{array}{|l}\text { Be-14 } \\
\mathrm{C}-18 \\
\mathrm{C}-19\end{array} \\
\end{array}$ & $\begin{array}{l}\text { Half-Life } \\
4.35 \mathrm{~ms} \\
95 \mathrm{~ms} \\
46 \mathrm{~ms}\end{array}$ & Parent & Half-Life & Parent & Half-Life \\
\hline $\mathrm{Na}-22$ & $2.6019 y$ & $\begin{array}{l}\mathrm{Al}-23 \\
\mathrm{Mg}-22\end{array}$ & $\begin{array}{l}0.47 \mathrm{~s} \\
3.857 \mathrm{~s}\end{array}$ & Al-22 & $70 \mathrm{~ms}$ & Si-22 & $6 \mathrm{~ms}$ & & \\
\hline $\mathrm{Na}-24$ & $14.959 \mathrm{~h}$ & $\mathrm{Ne}-24$ & $3.38 \mathrm{~m}$ & $\begin{array}{l}F-24 \\
F-25\end{array}$ & $\begin{array}{l}0.34 \mathrm{~s} \\
59 \mathrm{~ms}\end{array}$ & $0-24$ & $61 \mathrm{~ms}$ & & \\
\hline$A 1-28$ & $2.2414 \mathrm{~m}$ & $M g-28$ & $20.9 \mathrm{~h}$ & $\begin{array}{l}\mathrm{Na}-28 \\
\mathrm{Na}-29 \mathrm{~m}\end{array}$ & $\begin{array}{l}30.5 \mathrm{~ms} \\
44.9 \mathrm{~ms}\end{array}$ & $\begin{array}{l}\mathrm{Ne}-28 \\
\mathrm{Ne}-29\end{array}$ & $\begin{array}{l}17 \mathrm{~ms} \\
0.2 \mathrm{~s}\end{array}$ & & \\
\hline $\mathrm{Si}-31$ & $157.3 \mathrm{~m}$ & Al-31 & $644 \mathrm{~ms}$ & $\begin{array}{l}\mathrm{Mg}-31 \\
\mathrm{Mg}-32\end{array}$ & $\begin{array}{l}230 \mathrm{~ms} \\
120 \mathrm{~ms}\end{array}$ & $\begin{array}{l}\mathrm{Na}-31 \\
\mathrm{Na}-32 \\
\mathrm{Na}-32 \\
\mathrm{Na}-33\end{array}$ & $\begin{array}{l}17 \mathrm{~ms} \\
13.2 \mathrm{~ms} \\
13.2 \mathrm{~ms} \\
8.2 \mathrm{~ms}\end{array}$ & & \\
\hline $\mathrm{P}-32$ & $14.262 d$ & $\mathrm{Si}-32$ & $150 y$ & Al-32 & $33 \mathrm{~ms}$ & $\begin{array}{l}\mathrm{Mg}-32 \\
\mathrm{Mg}-33\end{array}$ & $\begin{array}{l}120 \mathrm{~ms} \\
90 \mathrm{~ms}\end{array}$ & $\begin{array}{l}\mathrm{Na}-32 \\
\mathrm{Na}-33 \\
\mathrm{Na}-33 \\
\mathrm{Na}-34\end{array}$ & $\begin{array}{l}13.2 \mathrm{~ms} \\
8.2 \mathrm{~ms} \\
8.2 \mathrm{~ms} \\
5.5 \mathrm{~ms} \\
\end{array}$ \\
\hline $\mathrm{Cl}-38$ & $37.24 \mathrm{~m}$ & $S-38$ & $170.3 \mathrm{~m}$ & $\begin{array}{l}\mathrm{P}-38 \\
\mathrm{P}-39\end{array}$ & $\begin{array}{l}0.64 \mathrm{~s} \\
0.16 \mathrm{~s}\end{array}$ & & & & \\
\hline $\mathrm{K}-42$ & $12.36 \mathrm{~h}$ & $\operatorname{Ar42}$ & $32.9 y$ & $\mathrm{Cl}-42$ & $6.8 \mathrm{~s}$ & $\begin{array}{l}S-42 \\
S-43\end{array}$ & $\begin{array}{l}0.56 \mathrm{~s} \\
220 \mathrm{~ms} \\
\end{array}$ & $\begin{array}{l}P-42 \\
P-43 \\
P-43\end{array}$ & $\begin{array}{l}110 \mathrm{~ms} \\
33 \mathrm{~ms} \\
33 \mathrm{~ms}\end{array}$ \\
\hline $\mathrm{Ca}-45$ & $162.61 d$ & $K-45$ & $17.3 \mathrm{~m}$ & Ar-45 & $21.48 s$ & $\begin{array}{l}\mathrm{Cl}-45 \\
\mathrm{Cl}-46\end{array}$ & $\begin{array}{l}400 \mathrm{~ms} \\
223 \mathrm{~ms}\end{array}$ & $S-45$ & $82 \mathrm{~ms}$ \\
\hline $\mathrm{Ca}-47$ & $4.536 d$ & $\begin{array}{l}K-47 \\
K-48\end{array}$ & $\begin{array}{l}17.5 \mathrm{~s} \\
6.8 \mathrm{~s} \\
\end{array}$ & Ar-47 & $700 \mathrm{~ms}$ & $\mathrm{Cl}-47$ & $>200 \mathrm{~ns}$ & & \\
\hline$M n-54$ & $312.3 d$ & & & & & & & & \\
\hline$M n-56$ & $2.5785 \mathrm{~h}$ & Cr-56 & $5.94 \mathrm{~m}$ & & & & & & \\
\hline Fe-59 & $44.503 d$ & $M n-59$ & $4.6 \mathrm{~s}$ & Cr-59 & $0.74 \mathrm{~s}$ & $V-59$ & $130 \mathrm{~ms}$ & & \\
\hline $\mathrm{Co}-60$ & $5.2714 y$ & $\mathrm{Fe}-60$ & $1.5 e 6 y$ & $\begin{array}{l}M n-60 \\
M n-60 m\end{array}$ & $\begin{array}{l}51 \mathrm{~s} \\
1.77 \mathrm{~s}\end{array}$ & $\begin{array}{l}\mathrm{Mn}-60 \mathrm{~m} \\
\mathrm{Cr}-60\end{array}$ & $\begin{array}{l}1.77 \mathrm{~s} \\
0.57 \mathrm{~s}\end{array}$ & & \\
\hline $\mathrm{Ni}-65$ & $2.5172 \mathrm{~h}$ & $\mathrm{Co}-65$ & $1.2 \mathrm{~s}$ & $\mathrm{Fe}-65$ & $0.4 \mathrm{~s}$ & $\operatorname{Mn} 65$ & $110 \mathrm{~ms}$ & & \\
\hline $\mathrm{Cu}-64$ & $12.7 \mathrm{~h}$ & & & & & & & & \\
\hline$Z n-65$ & $244.26 d$ & $\mathrm{Ga}-65$ & $15.2 \mathrm{~m}$ & Ge-65 & $30.9 \mathrm{~s}$ & As-65 & $0.19 \mathrm{~s}$ & $\mathrm{Se}-65$ & $<50 \mathrm{~ms}$ \\
\hline Sr-89 & $50.53 d$ & $R b-89$ & $15.15 \mathrm{~m}$ & Kr-89 & $3.15 \mathrm{~m}$ & $\begin{array}{l}\text { Br-89 } \\
\text { Br }-90\end{array}$ & $\begin{array}{l}4.348 \mathrm{~s} \\
1.91 \mathrm{~s}\end{array}$ & $\begin{array}{l}\mathrm{Se}-89 \\
\mathrm{Se}-91\end{array}$ & $\begin{array}{l}0.41 \mathrm{~s} \\
0.27 \mathrm{~s}\end{array}$ \\
\hline Sr -90 & $28.78 y$ & $\begin{array}{l}R b-90 \\
R b-90 m\end{array}$ & $\begin{array}{l}158 \mathrm{~s} \\
258 \mathrm{~s}\end{array}$ & $\begin{array}{l}\text { Kr-90 } \\
R b-90 m\end{array}$ & $\begin{array}{l}3.32 \mathrm{~m} \\
258 \mathrm{~s}\end{array}$ & $\begin{array}{l}\text { Br }-90 \\
\text { Br }-91\end{array}$ & $\begin{array}{l}1.91 \mathrm{~s} \\
0.541 \mathrm{~s}\end{array}$ & $\begin{array}{l}\mathrm{Se}-91 \\
\mathrm{Se}-91\end{array}$ & $\begin{array}{l}0.27 \mathrm{~s} \\
0.27 \mathrm{~s}\end{array}$ \\
\hline Sr-91 & $9.63 \mathrm{~h}$ & $R b-92$ & $4.492 s$ & $\begin{array}{l}\text { Kr-91 } \\
K r-92 \\
K r-92 \\
K r-93\end{array}$ & $\begin{array}{l}8.57 \mathrm{~s} \\
1.84 \mathrm{~s} \\
1.84 \mathrm{~s} \\
1.286 \mathrm{~s}\end{array}$ & $\begin{array}{l}\mathrm{Br}-91 \\
\mathrm{Br}-92 \\
\mathrm{Br}-92 \\
\mathrm{Br}-93 \\
\mathrm{Br}-92 \\
\mathrm{Br}-93 \\
\mathrm{Br}-93 \\
\mathrm{Br}-94\end{array}$ & $\begin{array}{l}0.541 \mathrm{~s} \\
0.343 \mathrm{~s} \\
0.343 \mathrm{~s} \\
102 \mathrm{~ms} \\
0.343 \mathrm{~s} \\
102 \mathrm{~ms} \\
102 \mathrm{~ms} \\
70 \mathrm{~ms} \\
\end{array}$ & $\mathrm{Se}-91$ & $0.27 \mathrm{~s}$ \\
\hline Sr-93 & $7.423 \mathrm{~m}$ & $\begin{array}{l}R b-93 \\
R b-94\end{array}$ & $2.702 \mathrm{~s}$ & $\begin{array}{l}\text { Kr-93 } \\
\text { Kr-94 } \\
\text { Kr-94 }\end{array}$ & $\begin{array}{l}1.286 \mathrm{~s} \\
0.2 \mathrm{~s} \\
0.2 \mathrm{~s}\end{array}$ & $\begin{array}{l}\mathrm{Br}-93 \\
\mathrm{~B} r-94 \\
\mathrm{~B} r-94 \\
\mathrm{~B} r-94\end{array}$ & $\begin{array}{l}102 \mathrm{~ms} \\
70 \mathrm{~ms} \\
70 \mathrm{~ms} \\
70 \mathrm{~ms}\end{array}$ & & \\
\hline
\end{tabular}

Table 4: Parent decay chains. 
UCRL-TR-203529

Appendix A: Cross-Sections for Neutron Transport

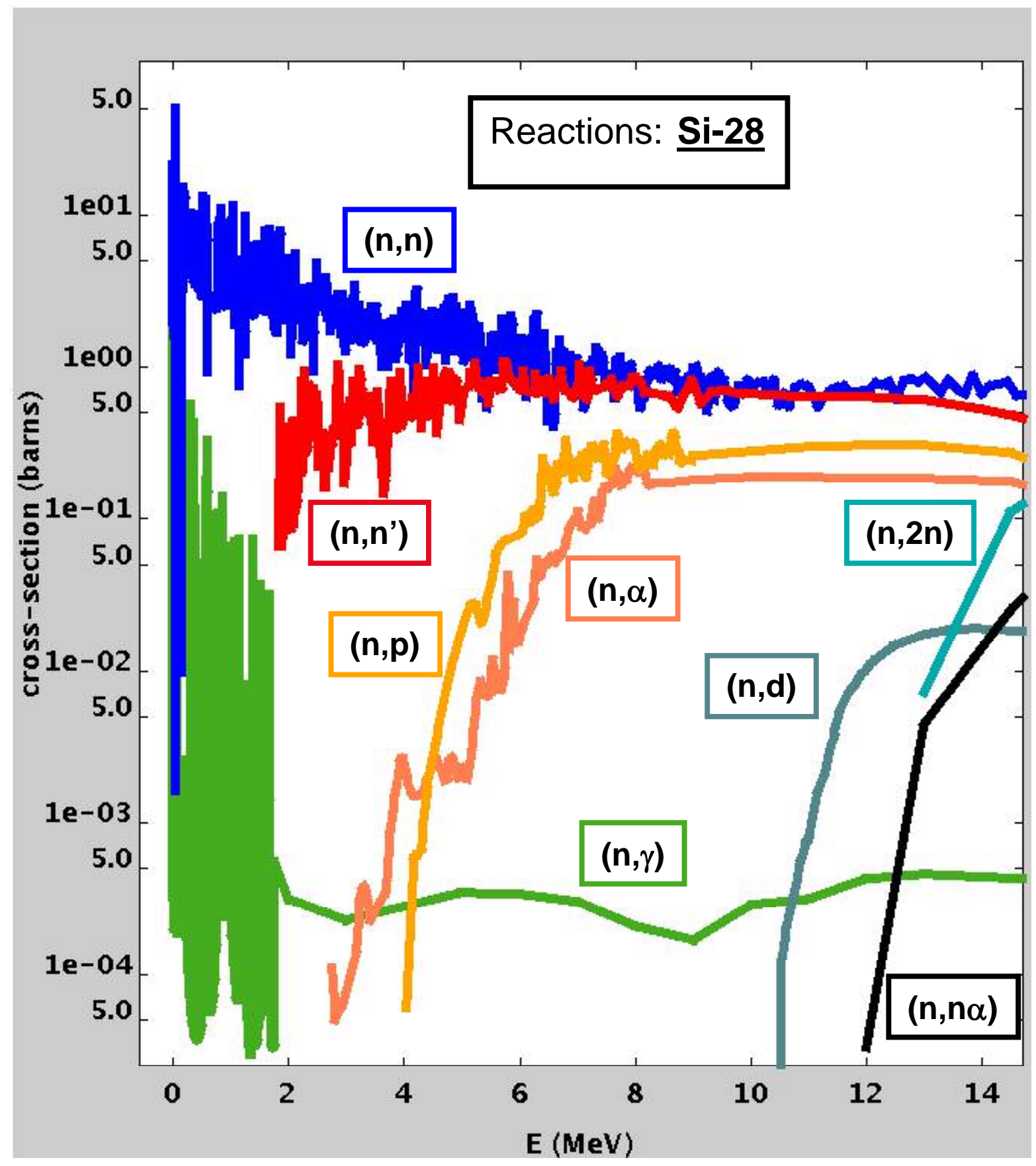

Figure 25: (Appendix A) Neutron cross-sections for target ${ }^{28}$ Si from ENDF/B-VI.8. 
UCRL-TR-203529

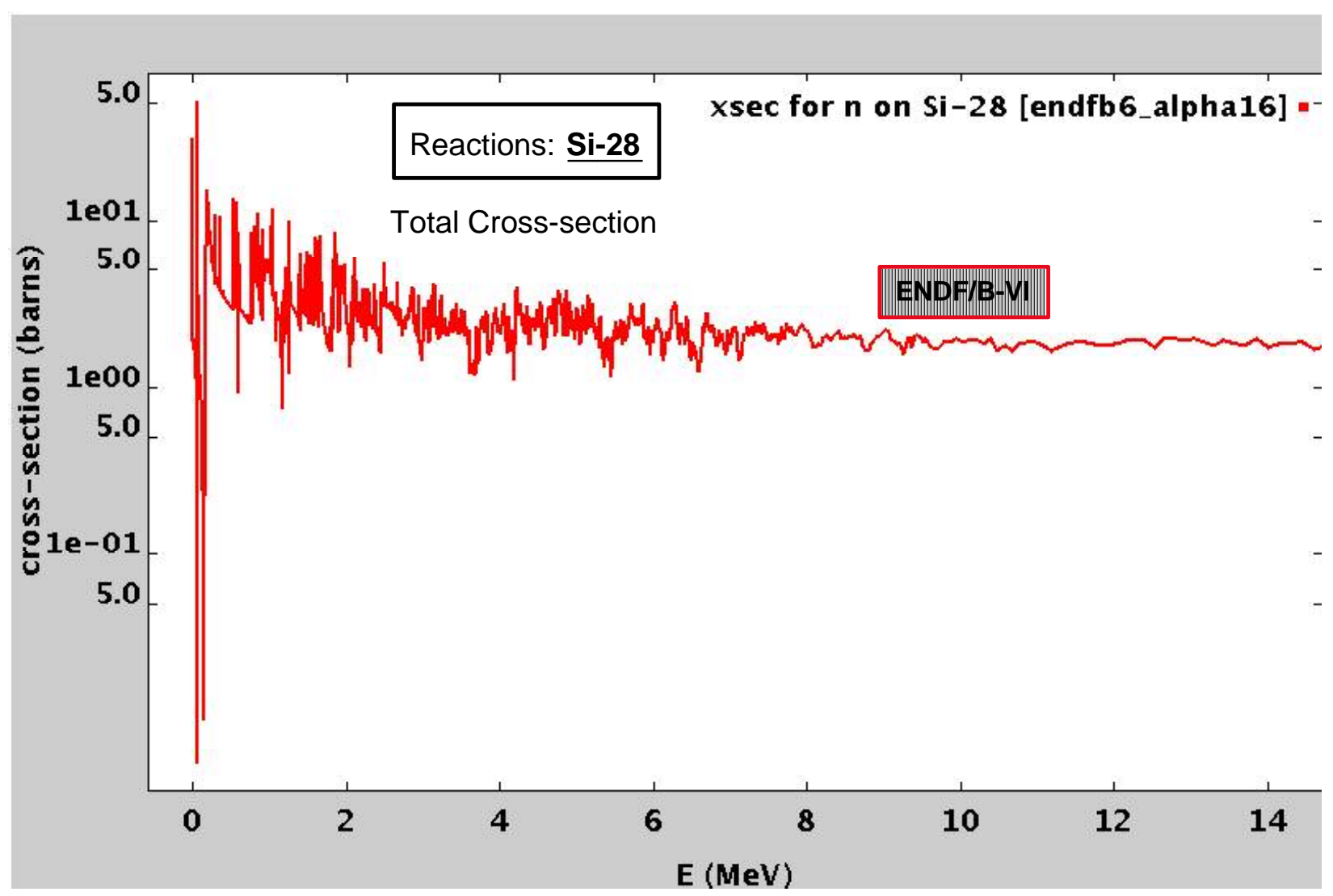

Figure 26: (Appendix A) Total Cross Section for neutrons incident on ${ }^{28} \mathrm{Si}$, from ENDF/B-VI.8 
UCRL-TR-203529

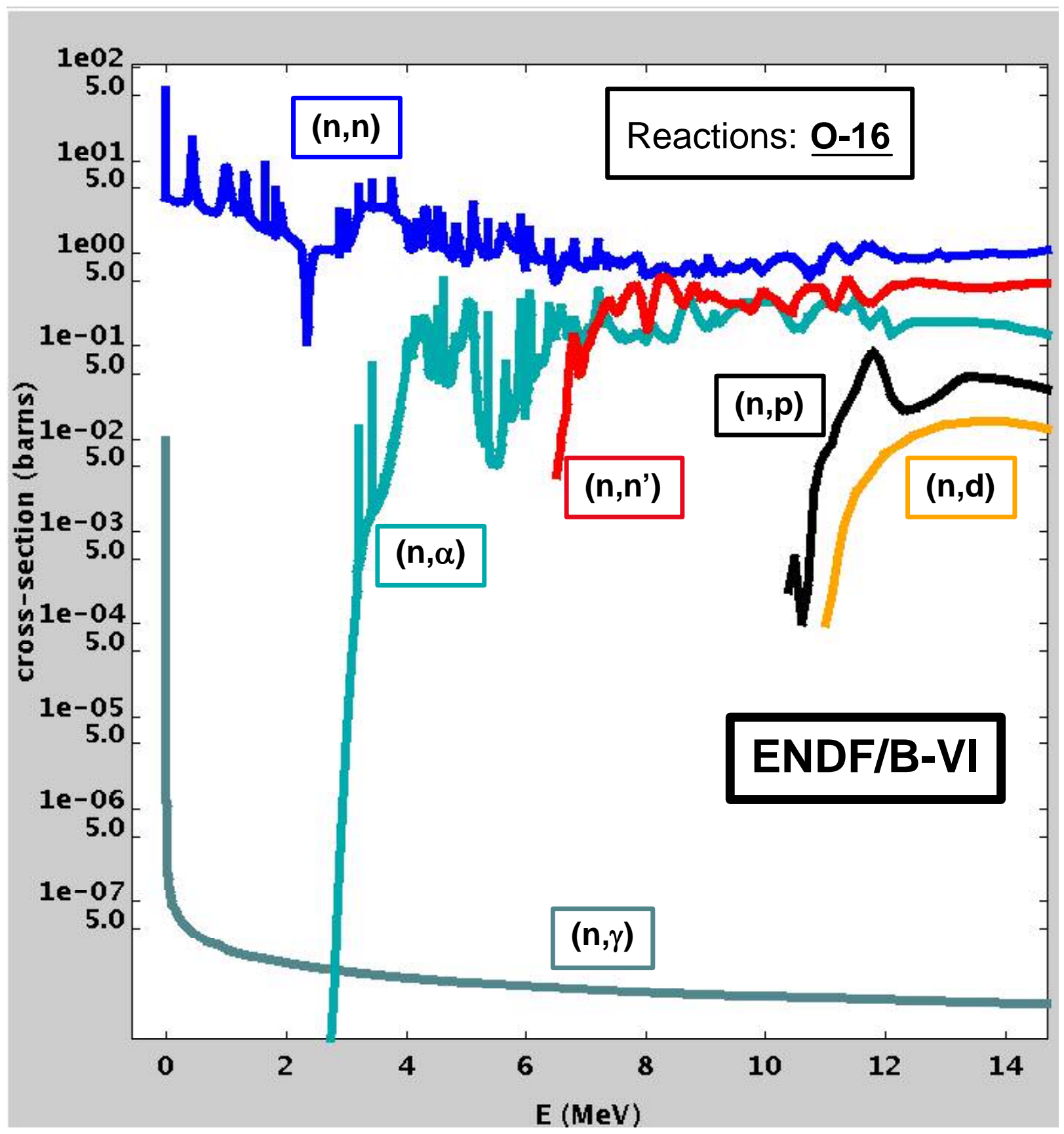

Figure 27: (Appendix A) Neutron cross-sections for target ${ }^{16} \mathrm{O}$ from ENDF/B-VI.8. 
UCRL-TR-203529

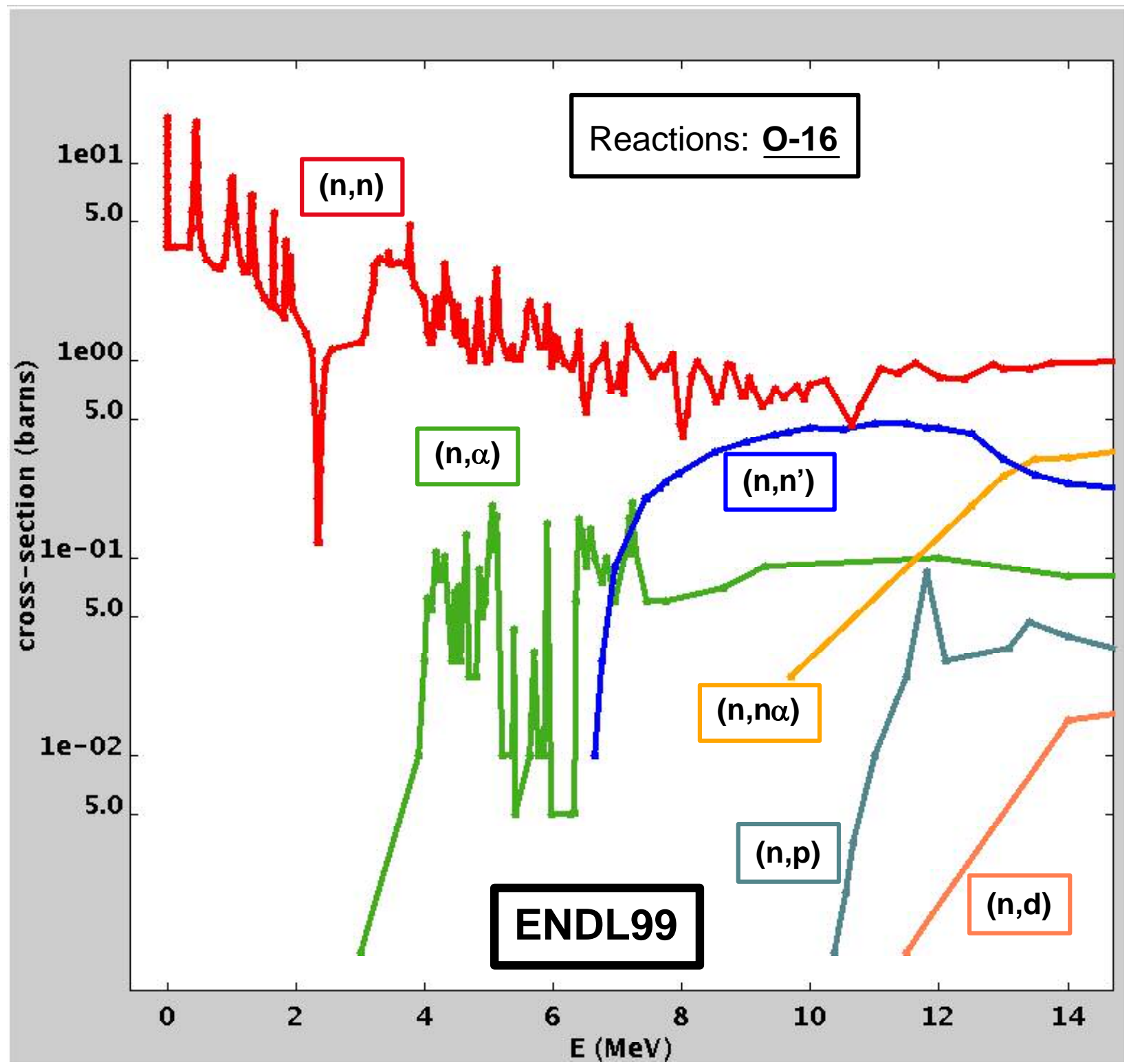

Figure 28: (Appendix A) Neutron cross-sections for target ${ }^{16} \mathrm{O}$ from ENDL99. 
UCRL-TR-203529

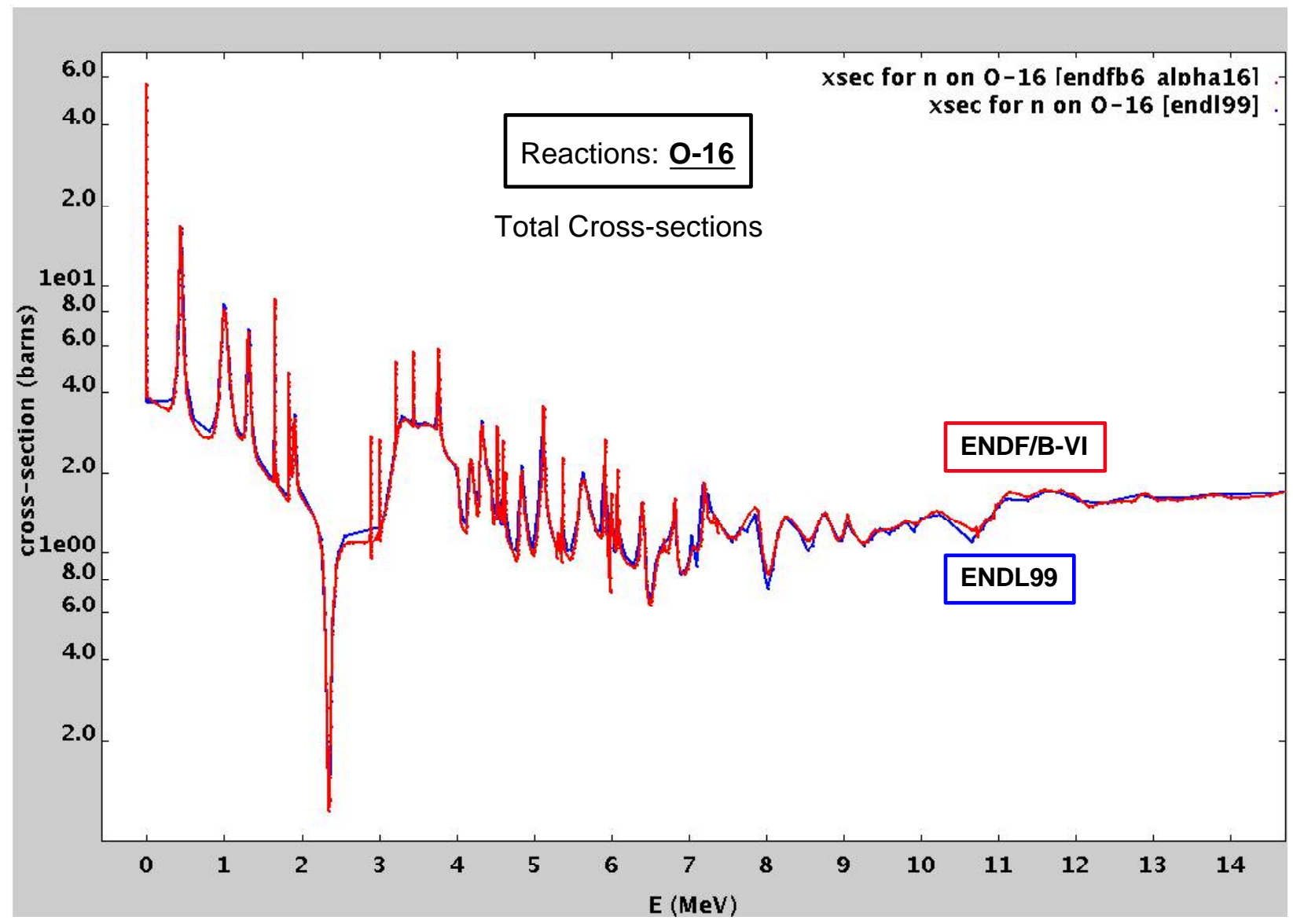

Figure 29: (Appendix A) Total Cross Section for neutrons incident on ${ }^{16} \mathrm{O}$, from both ENDF/B-VI.8 and ENDL99. 
UCRL-TR-203529

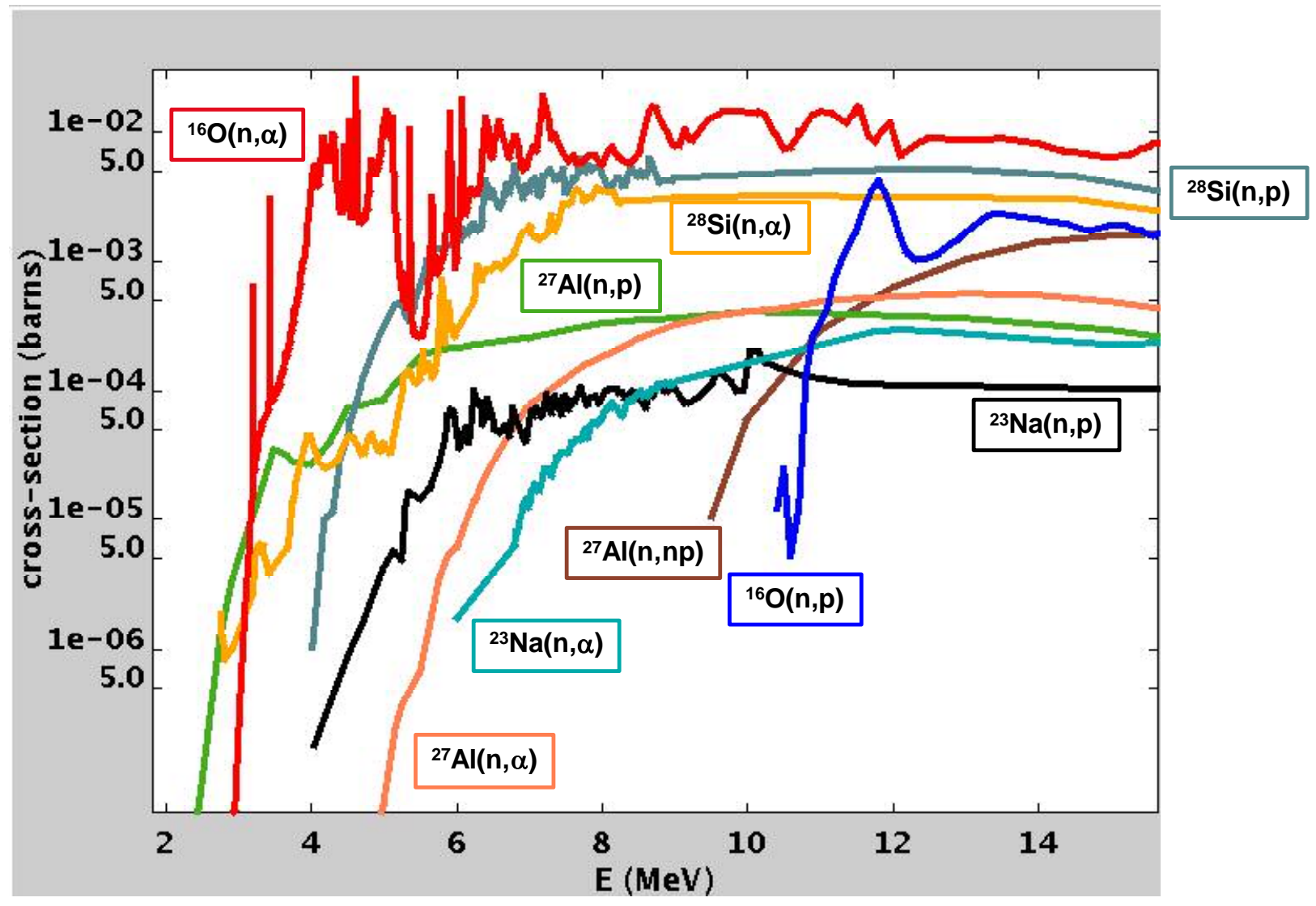

Figure 30: (Appendix A) ENDF/B-VI cross-sections for dominant transmutation reactions in granite, WEIGHTED BY THE ATOMIC COMPOSITIONS IN GRANITE. The vertical scale is the cross section multiplied by the number of atoms/barn-cm of the target nuclide. 
UCRL-TR-203529

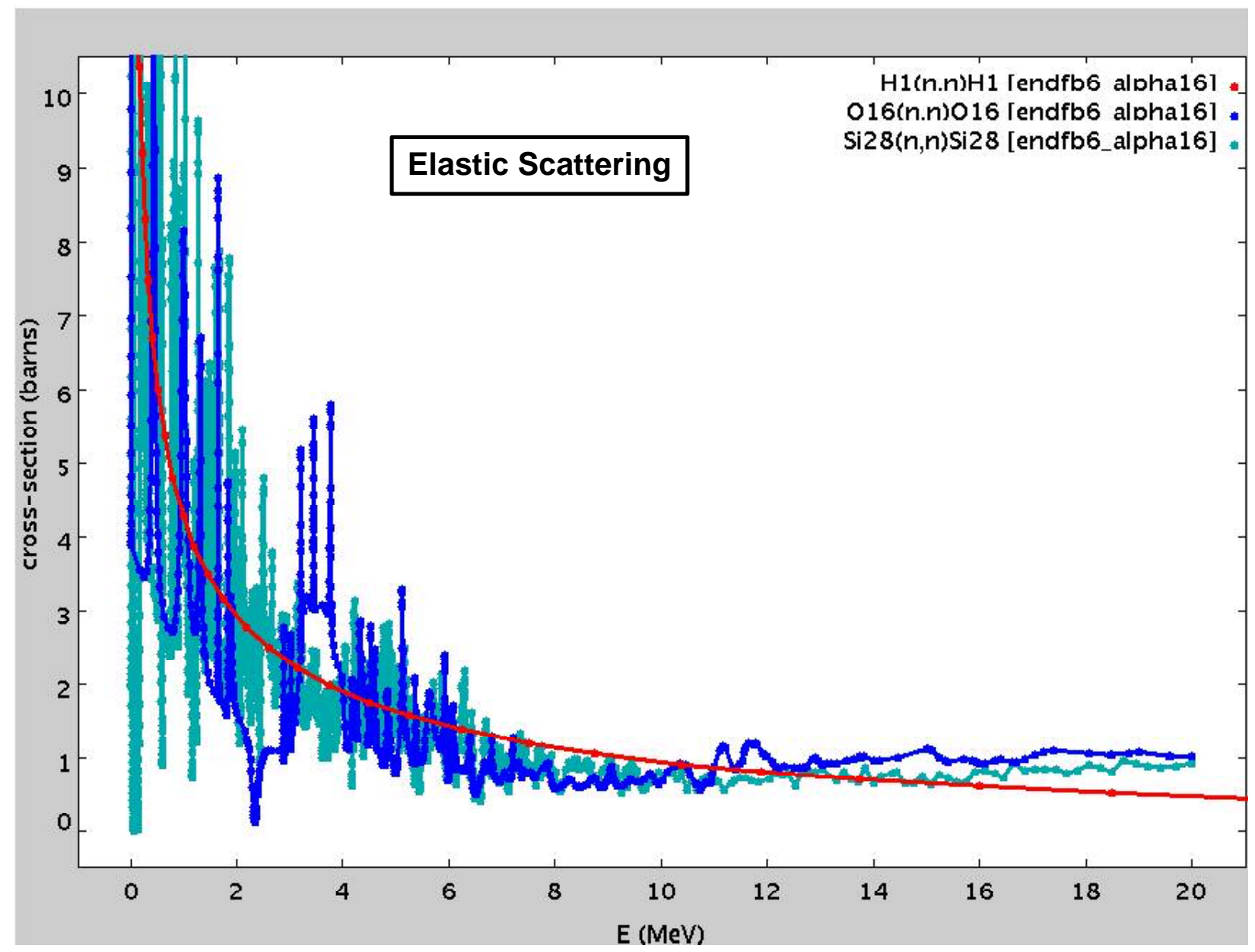

Figure 31: (Appendix A) Neutron elastic cross-sections for ${ }^{1} \mathrm{H},{ }^{16} \mathrm{O}$, and ${ }^{28} \mathrm{Si}$. 


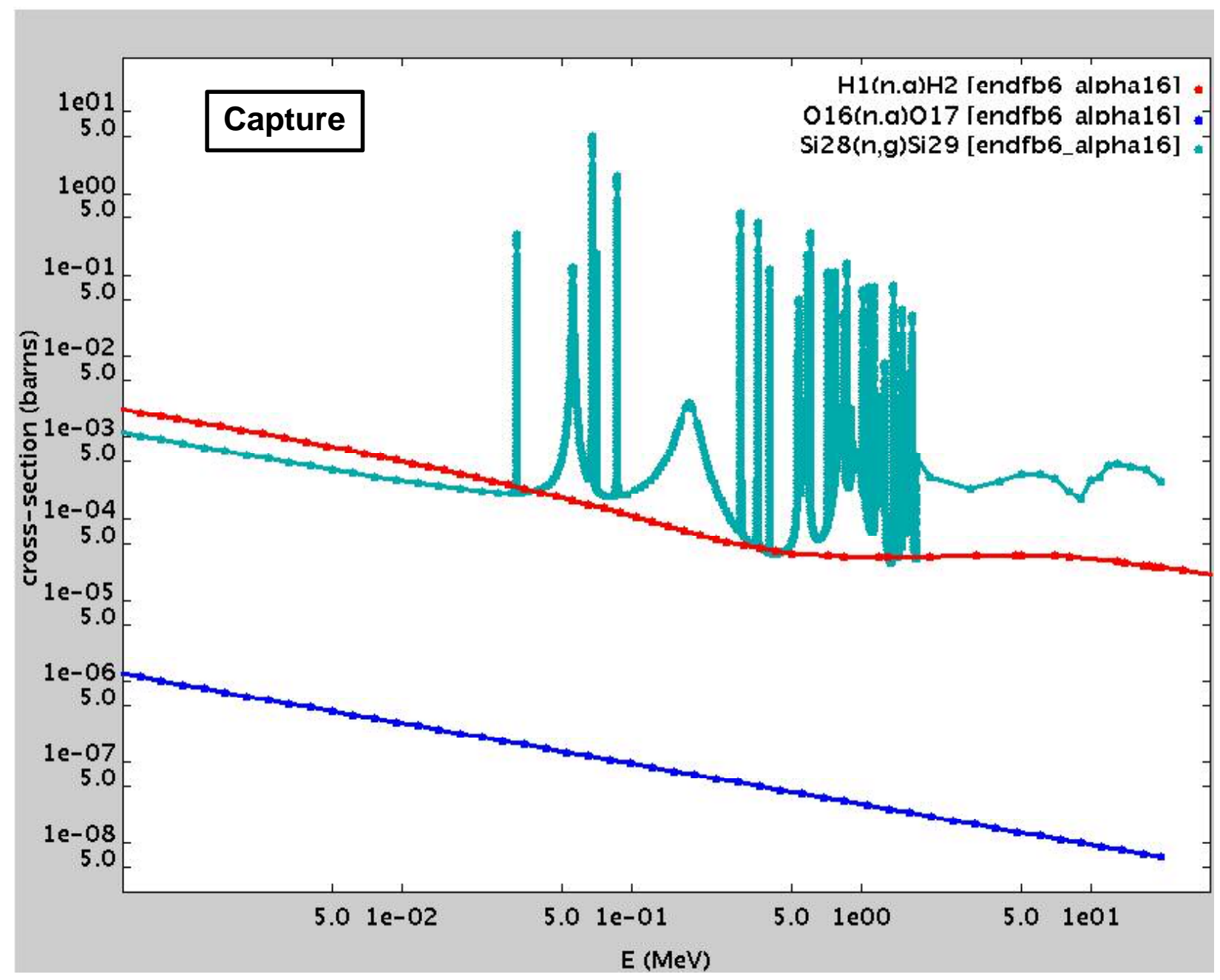

Figure 32: (Appendix A) Capture cross-sections for ${ }^{1} \mathrm{H},{ }^{16} \mathrm{O}$, and ${ }^{28} \mathrm{Si}$. 
UCRL-TR-203529

\section{Appendix B: Radionuclide Production Cross-Sections}
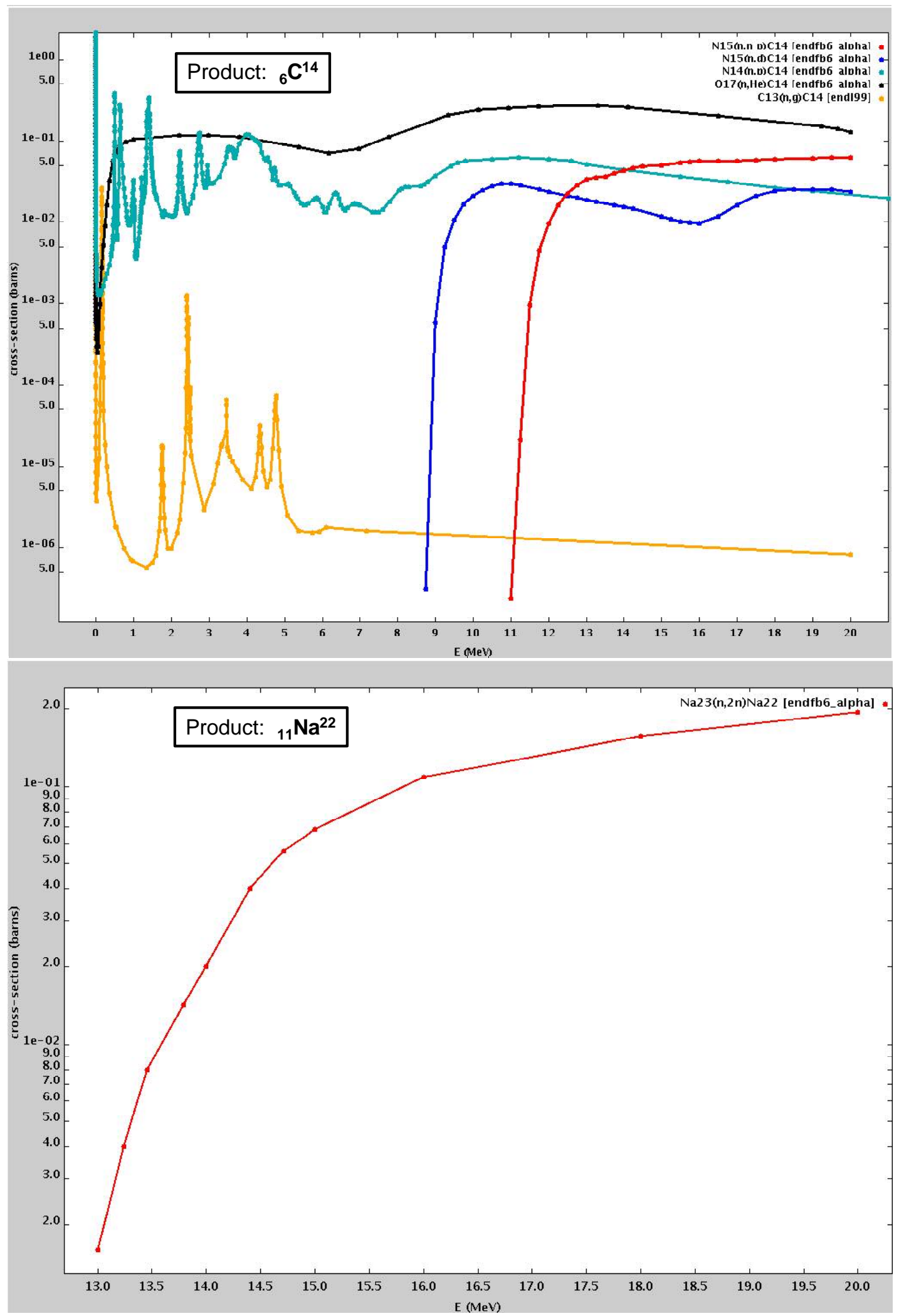
UCRL-TR-203529

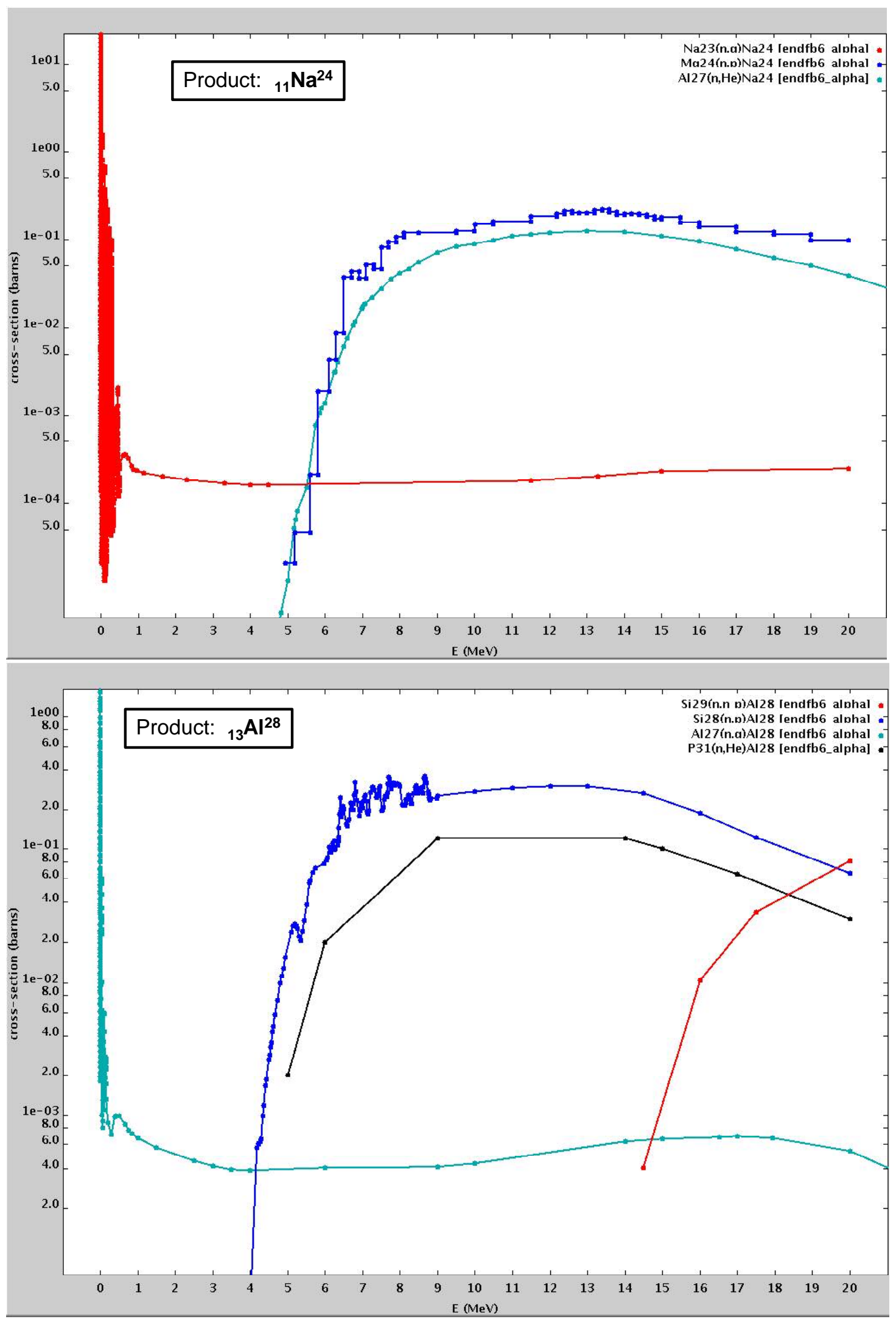

Page 47 of 64 
UCRL-TR-203529
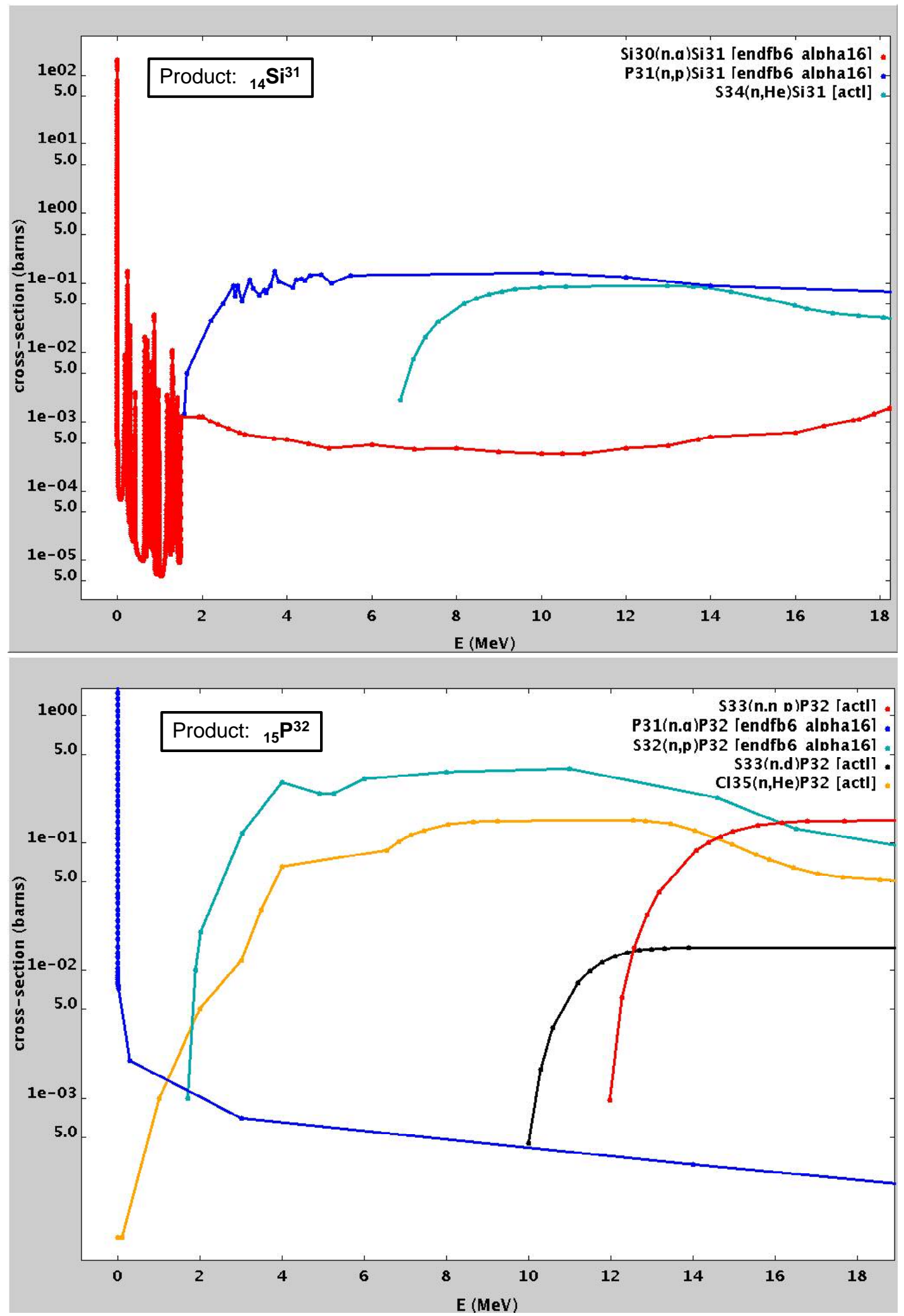
UCRL-TR-203529

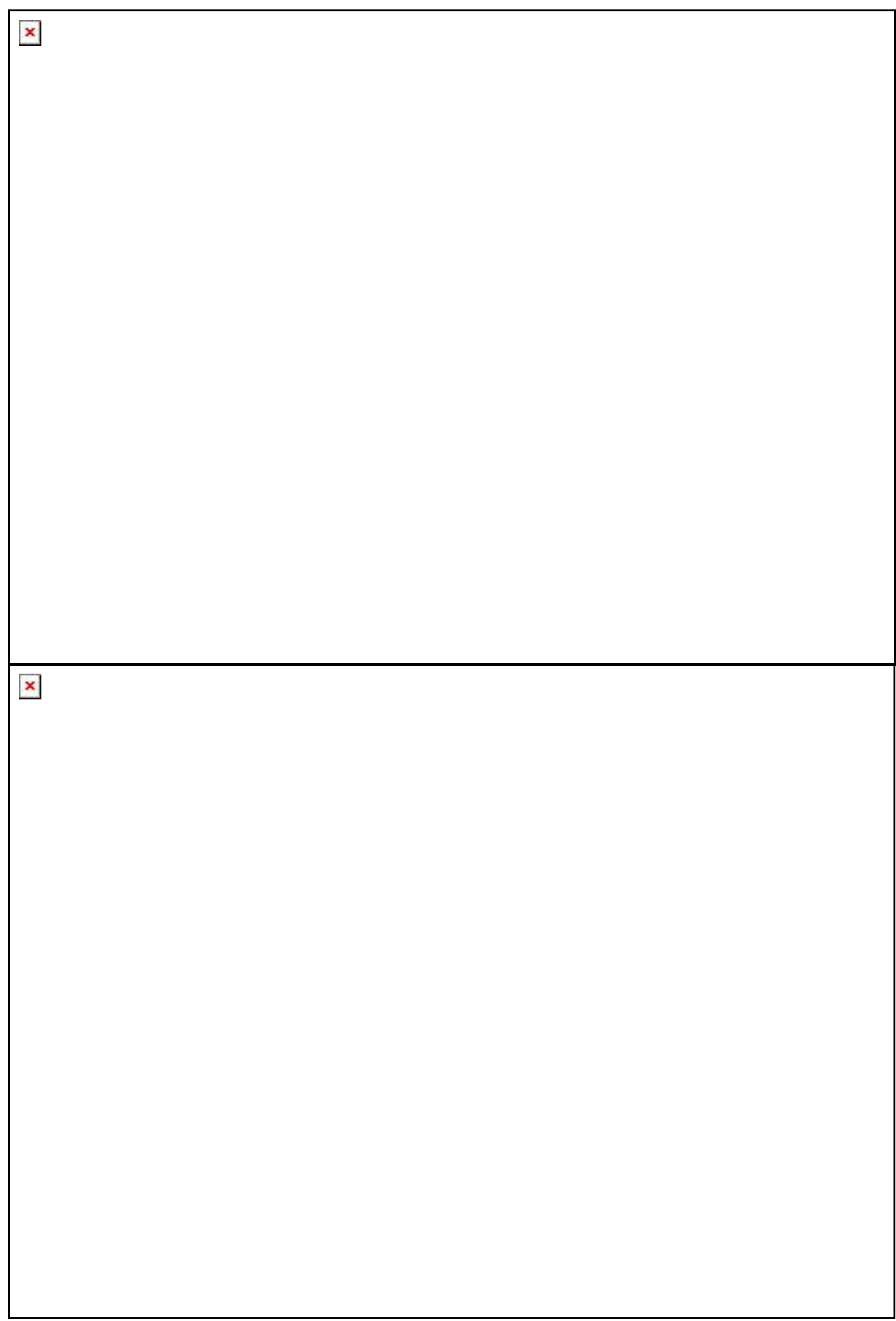

Page 49 of 64 
UCRL-TR-203529
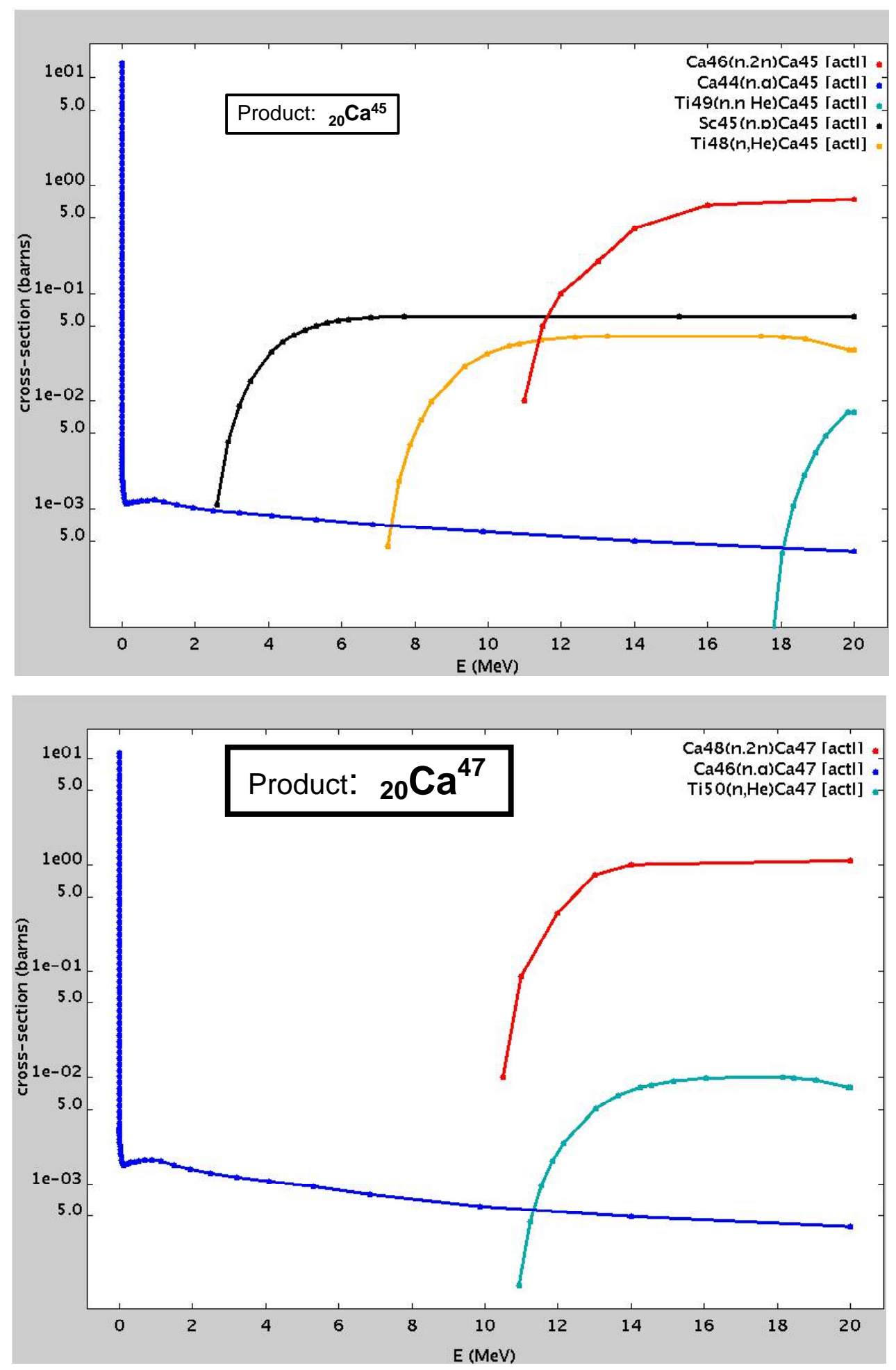

Page 50 of 64 
UCRL-TR-203529

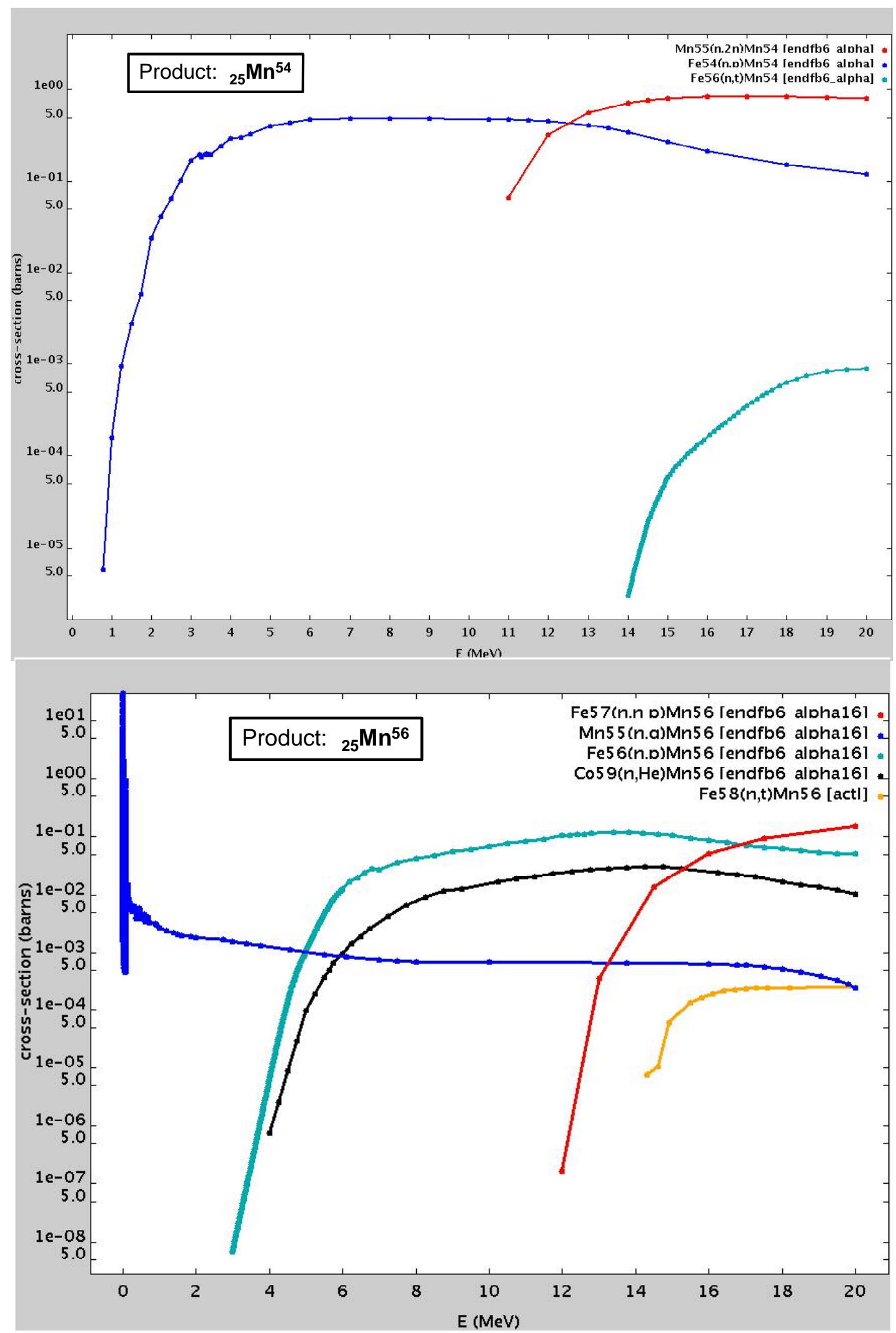

Page 51 of 64 
UCRL-TR-203529
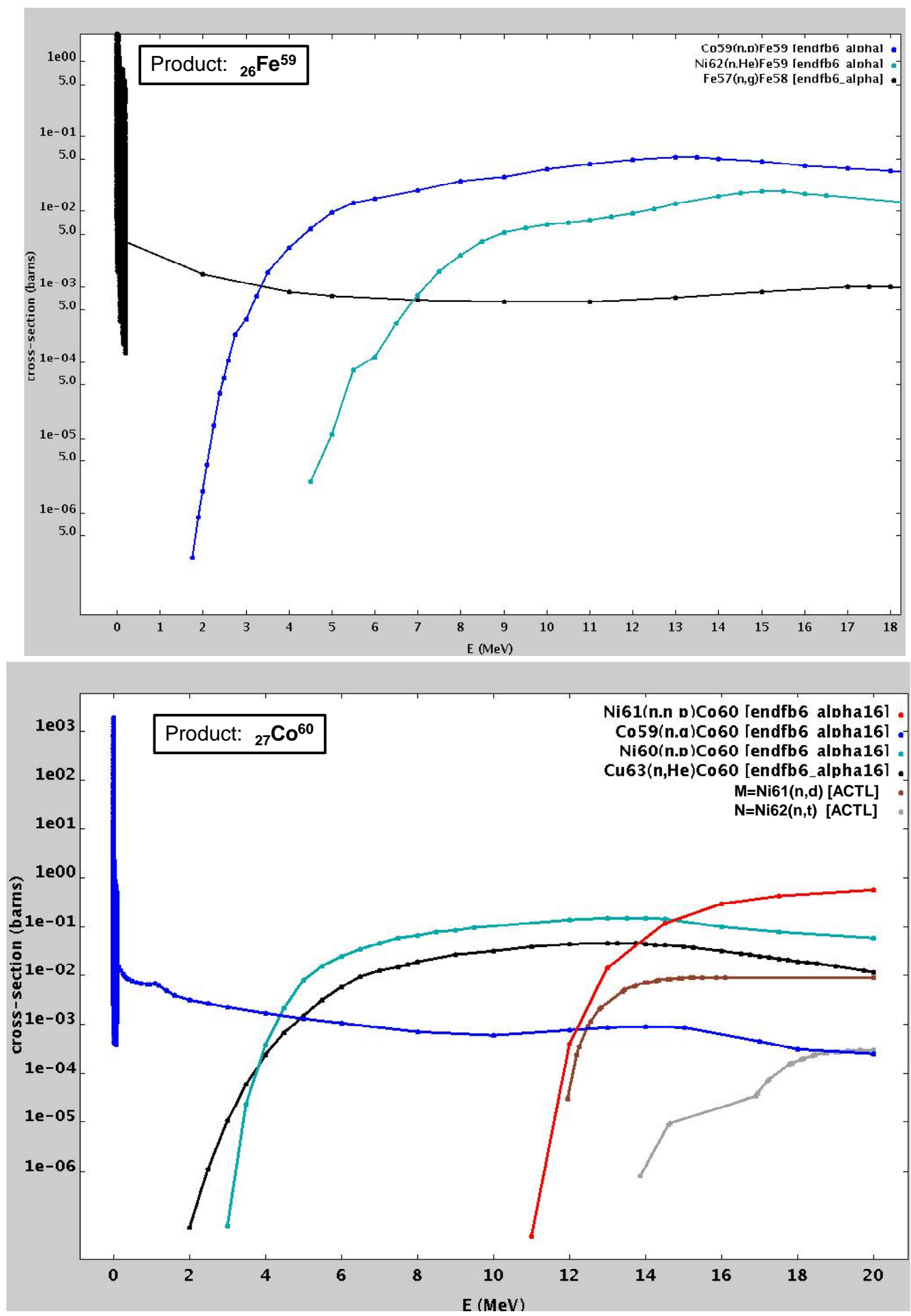

Page 52 of 64 
UCRL-TR-203529

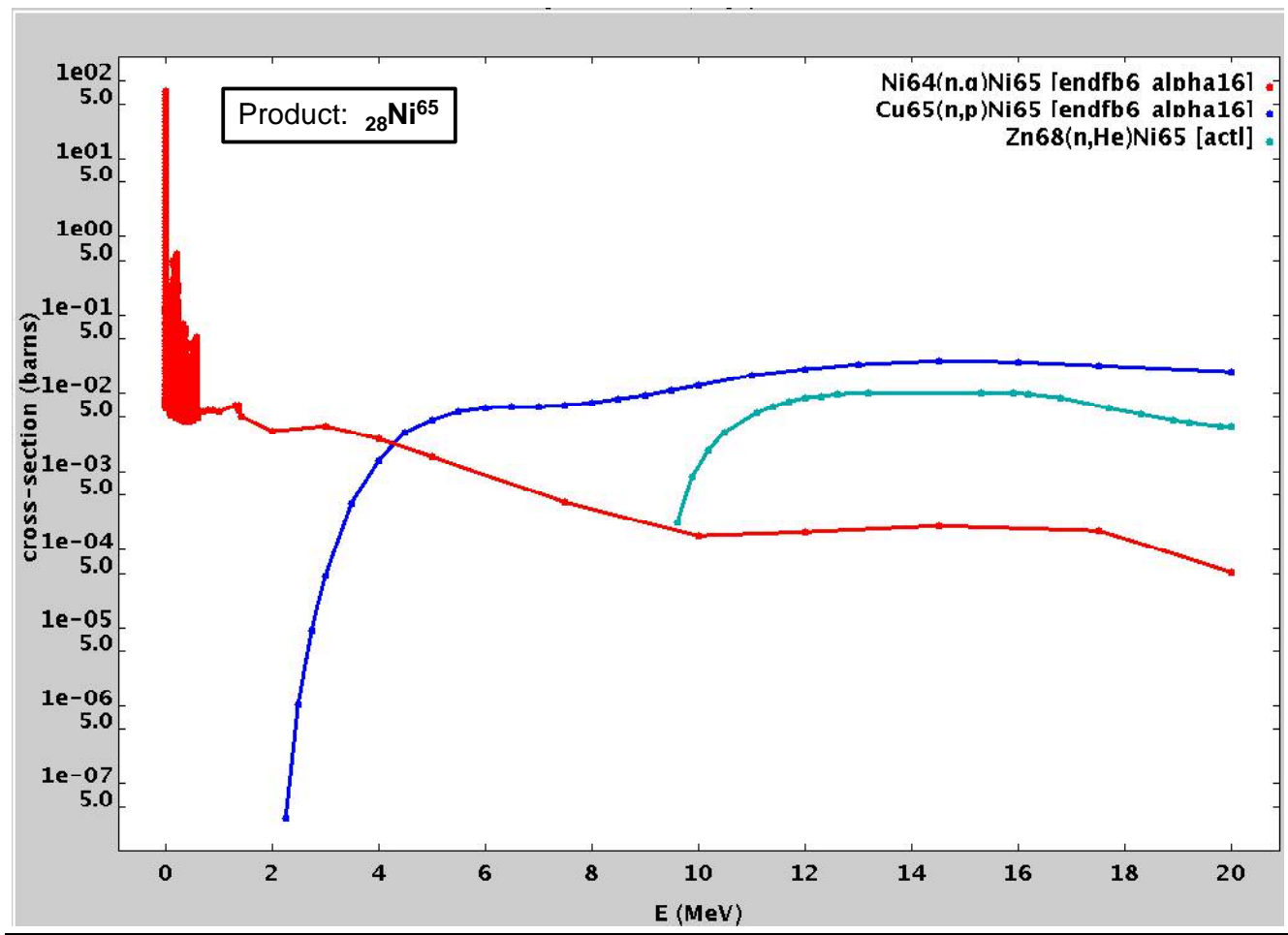

$x$ 
UCRL-TR-203529
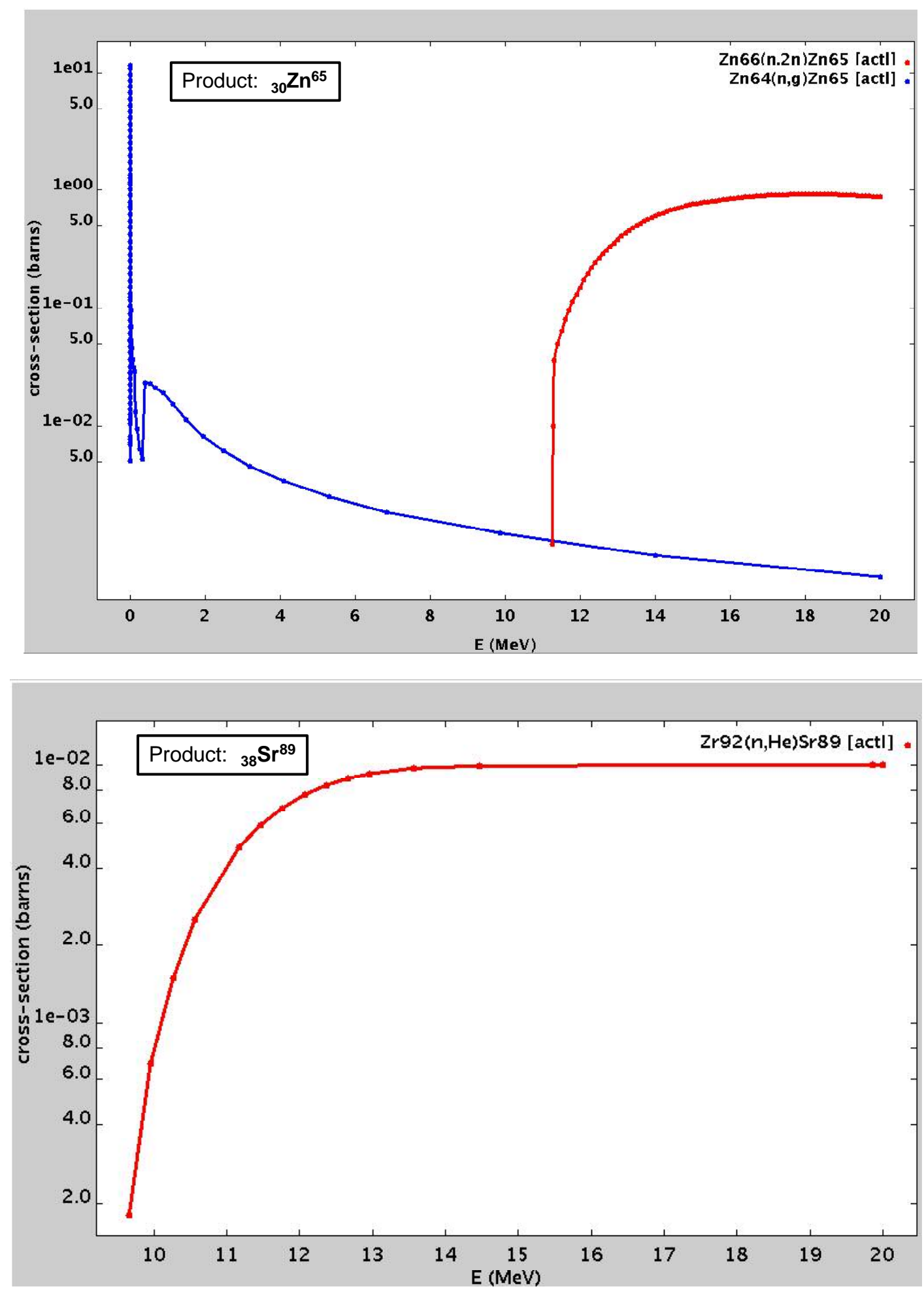

Page 54 of 64 
UCRL-TR-203529
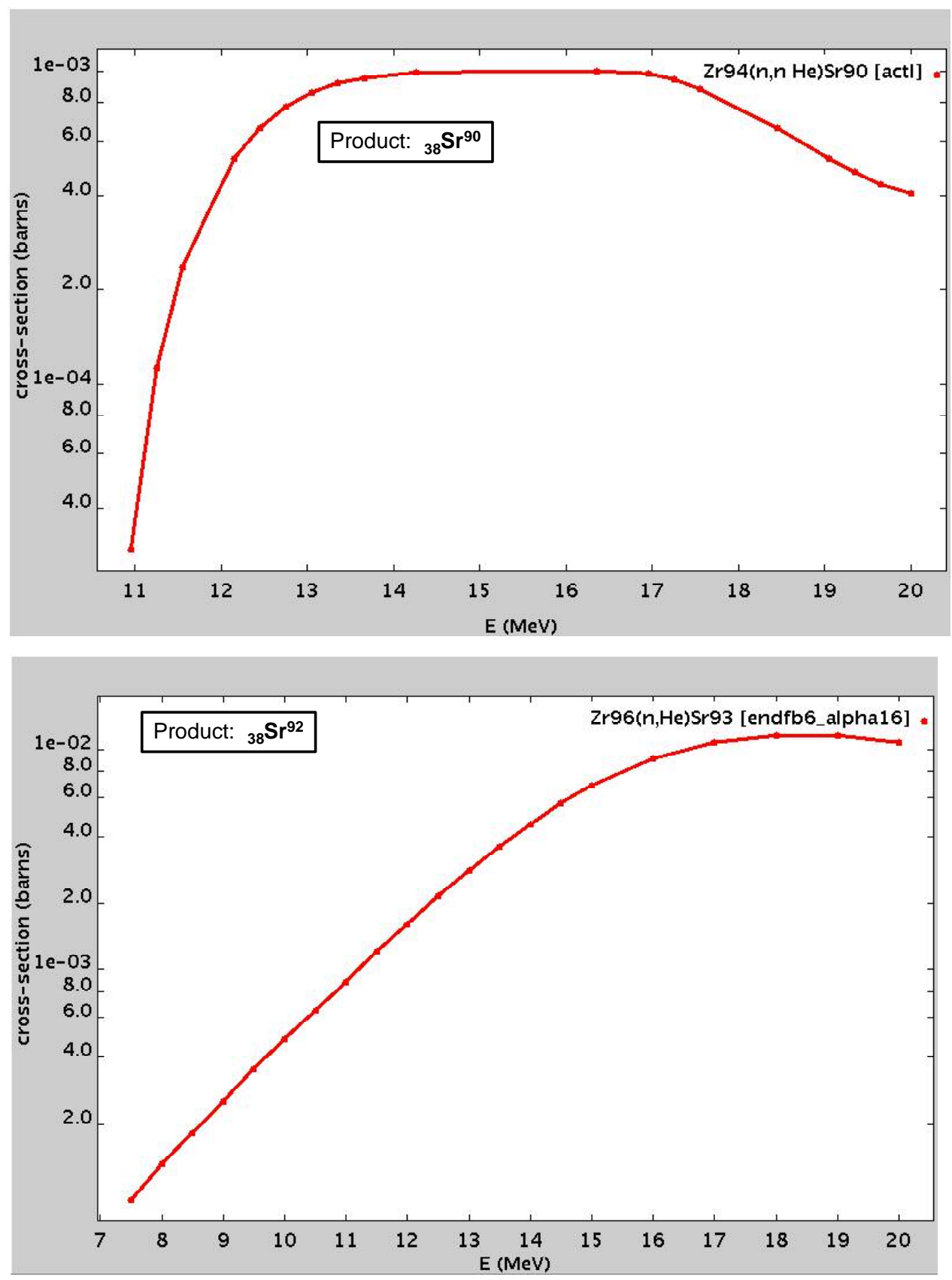

Page 55 of 64 
UCRL-TR-203529

\section{Appendix C: Cross Section Sources for Radionuclide Production}

\begin{tabular}{|c|c|c|c|}
\hline Product & Library & Target & $\underline{\mathrm{R} \times \mathrm{n}}$ \\
\hline $\mathrm{H}-3$ & $\overline{\mathrm{LLNL} / \mathrm{ACTL}}$ & $\mathrm{H}-2$ & $\overline{(n, g)}$ \\
\hline $\mathrm{H}-3$ & ENDFb 6 & $\mathrm{H}-2$ & $(n, g)$ \\
\hline $\mathrm{H}-3$ & LLNL/ACTL & $\mathrm{He}-3$ & $(n, p)$ \\
\hline $\mathrm{H}-3$ & ENDFb 6 & $\mathrm{He}-3$ & $(n, p)$ \\
\hline $\mathrm{He}-6$ & LLNL/ACTL & Li-6 & $(n, p)$ \\
\hline $\mathrm{He}-6$ & ENDFb 6 & Li-6 & $(n, p)$ \\
\hline $\mathrm{He}-6$ & ENDFb 6 & $\mathrm{Li}-7$ & $(n, d)$ \\
\hline $\mathrm{He}-6$ & LLNL/ACTL & $\mathrm{Be}-9$ & $(n, a)$ \\
\hline $\mathrm{He}-6$ & ENDFb 6 & $\mathrm{Be}-9$ & $(n, a)$ \\
\hline $\mathrm{Li}-8$ & ENDFb5/532dos & Li-7 & $(n, g)$ \\
\hline $\mathrm{Li}-8$ & LLNL/ACTL & Li-7 & $(n, g)$ \\
\hline Li-8 & ENDL94 & Li-7 & $(n, g)$ \\
\hline $\mathrm{Li}-8$ & ENDFb 6 & $\mathrm{Li}-7$ & $(n, g)$ \\
\hline $\mathrm{Li}-8$ & LLNL/ACTL & $\mathrm{Be}-9$ & $(n, d)$ \\
\hline $\mathrm{Li}-8$ & ENDFb 6 & $\mathrm{Be}-9$ & $(n, d)$ \\
\hline $\mathrm{Li}-8$ & LLNL/ACTL & $B-11$ & $(n, a)$ \\
\hline $\mathrm{Li}-8$ & ENDFb 6 & $B-11$ & $(n, a)$ \\
\hline Li-9 & LLNL/ACTL & $\mathrm{Be}-9$ & $(n, p)$ \\
\hline Li-9 & ENDFb 6 & $\mathrm{Be}-9$ & $(n, p)$ \\
\hline $\mathrm{He}-4$ & LLNL/ACTL & $\mathrm{Be}-9$ & $(n, 2 n)$ \\
\hline $\mathrm{Be}-8$ & ENDFb 6 & $\mathrm{Be}-9$ & $(n, 2 n)$ \\
\hline $\mathrm{Be}-8$ & ENDFb 6 & $B-10$ & $(n, t)$ \\
\hline $\mathrm{Be}-10$ & ENDFb 6 & $B-11$ & $(n, n p)$ \\
\hline $\mathrm{Be}-10$ & LLNL/ACTL & $\mathrm{Be}-9$ & $(n, g)$ \\
\hline $\mathrm{Be}-10$ & ENDFb 6 & $\mathrm{Be}-9$ & $(n, g)$ \\
\hline $\mathrm{Be}-10$ & ENDFb 6 & $B-10$ & $(n, p)$ \\
\hline $\mathrm{Be}-10$ & LLNL/ACTL & $C-13$ & $(n, a)$ \\
\hline $\mathrm{Be}-11$ & LLNL/ACTL & $B-11$ & $(n, p)$ \\
\hline $\mathrm{Be}-11$ & ENDFb 6 & $B-11$ & $(n, p)$ \\
\hline$B-9$ & ENDL94 & $B-10$ & $(n, 2 n)$ \\
\hline$B-12$ & LLNL/ACTL & $B-11$ & $(n, g)$ \\
\hline$B-12$ & ENDL94 & $B-11$ & $(n, g)$ \\
\hline$B-12$ & ENDFb 6 & $B-11$ & $(n, g)$ \\
\hline$B-12$ & LLNL/ACTL & $C-12$ & $(n, p)$ \\
\hline$B-12$ & ENDFb 6 & $\mathrm{~N}-15$ & $(n, a)$ \\
\hline$B-13$ & LLNL/ACTL & $C-13$ & $(n, p)$ \\
\hline$C-14$ & ENDL 94 & $N-15$ & $(n, n p)$ \\
\hline$C-14$ & ENDFb 6 & $N-15$ & $(n, n p)$ \\
\hline$C-14$ & LLNL/ACTL & $C-13$ & $(n, g)$ \\
\hline$C-14$ & ENDL94 & $C-13$ & $(n, g)$ \\
\hline$C-14$ & ENDFb5/532dos & $\mathrm{N}-\mathrm{I} 4$ & $(n, p)$ \\
\hline$C-14$ & LLNL/ACTL & $N-14$ & $(n, p)$ \\
\hline$C-14$ & ENDFb 6 & $\mathrm{~N}-14$ & $(n, p)$ \\
\hline$C-14$ & ENDFb 6 & $\mathrm{~N}-15$ & $(n, d)$ \\
\hline$C-14$ & LLNL/ACTL & $0-17$ & $(n, a)$ \\
\hline$C-14$ & ENDFb 6 & $0-17$ & $(n, a)$ \\
\hline$C-15$ & ENDFb 6 & $N-15$ & $(n, p)$ \\
\hline$N-13$ & LLNL/ACTL & $N-14$ & $(n, 2 n)$ \\
\hline$N-13$ & ENDL 94 & $N-14$ & $(n, 2 n)$ \\
\hline$N-13$ & ENDFb 6 & $N-14$ & $(n, 2 n)$ \\
\hline$N-16$ & ENDFb 6 & $0-17$ & $(n, n p)$ \\
\hline$N-16$ & ENDL 94 & $\mathrm{~N}-15$ & $(n, g)$ \\
\hline$N-16$ & ENDFb 6 & $N-15$ & $(n, g)$ \\
\hline$N-16$ & ENDFb5/532dos & $0-I 6$ & $(n, p)$ \\
\hline$N-16$ & LLNL/ACTL & $0-16$ & $(n, p)$ \\
\hline$N-16$ & ENDFb 6 & $0-16$ & $(n, p)$ \\
\hline$N-16$ & ENDFb 6 & $0-17$ & $(n, d)$ \\
\hline$N-16$ & LLNL/ACTL & $F-19$ & $(n, a)$ \\
\hline$N-16$ & ENDFb 6 & $F-19$ & $(n, a)$ \\
\hline$N-17$ & ENDFb 6 & $0-17$ & $(n, p)$ \\
\hline $0-15$ & LLNL/ACTL & $0-16$ & $(n, 2 n)$ \\
\hline $0-15$ & ENDL94 & $0-16$ & $(n, 2 n)$ \\
\hline $0-19$ & LLNL/ACTL & $F-19$ & $(\mathrm{n}, \mathrm{p})$ \\
\hline
\end{tabular}

\begin{tabular}{|c|c|c|c|}
\hline $0-19$ & ENDFb 6 & $\mathrm{~F}-19$ & $(n, p)$ \\
\hline $\mathrm{F}-18$ & ENDFb5/532dos & $F-I 9$ & $(n, 2 n)$ \\
\hline$F-18$ & LLNL/ACTL & $\mathrm{F}-19$ & $(n, 2 n)$ \\
\hline$F-18$ & ENDL 94 & $\mathrm{~F}-19$ & $(n, 2 n)$ \\
\hline$F-18$ & ENDFb 6 & $\mathrm{~F}-19$ & $(n, 2 n)$ \\
\hline$F-20$ & LLNL/ACTL & $\mathrm{F}-19$ & $(n, g)$ \\
\hline$F-20$ & ENDL 94 & $\mathrm{~F}-19$ & $(n, g)$ \\
\hline$F-20$ & ENDFb 6 & $\mathrm{~F}-19$ & $(n, g)$ \\
\hline$F-20$ & LLNL/ACTL & $\mathrm{Na}-23$ & $(n, a)$ \\
\hline$F-20$ & ENDFb 6 & $\mathrm{Na}-23$ & $(n, a)$ \\
\hline$F-20$ & ENDFb 6 & Al-27 & $(n, 2 a)$ \\
\hline $\mathrm{Ne}-19$ & ENDL 94 & $\mathrm{Ne}-20$ & $(n, 2 n)$ \\
\hline $\mathrm{Ne}-23$ & LLNL/ACTL & $\mathrm{Na}-23$ & $(n, p)$ \\
\hline $\mathrm{Ne}-23$ & ENDFb 6 & $\mathrm{Na}-23$ & $(n, p)$ \\
\hline $\mathrm{Ne}-23$ & LLNL/ACTL & $M g-26$ & $(n, a)$ \\
\hline $\mathrm{Ne}-23$ & ENDFb 6 & $\mathrm{Al}-27$ & $(\mathrm{n}, \mathrm{pa})$ \\
\hline $\mathrm{Na}-22$ & LLNL/ACTL & $\mathrm{Na}-23$ & $(n, 2 n)$ \\
\hline $\mathrm{Na}-22$ & ENDL 94 & $\mathrm{Na}-23$ & $(n, 2 n)$ \\
\hline $\mathrm{Na}-22$ & ENDFb 6 & $\mathrm{Na}-23$ & $(n, 2 n)$ \\
\hline $\mathrm{Na}-24$ & LLNL/ACTL & $\mathrm{Na}-23$ & $(n, g)$ \\
\hline $\mathrm{Na}-24$ & ENDL 94 & $\mathrm{Na}-23$ & $(n, g)$ \\
\hline $\mathrm{Na}-24$ & ENDFb 6 & $\mathrm{Na}-23$ & $(n, g)$ \\
\hline $\mathrm{Na}-24$ & ENDFb5/532dos & $\mathrm{Mg}-24$ & $(n, p)$ \\
\hline $\mathrm{Na}-24$ & LLNL/ACTL & $\mathrm{Mg}-24$ & $(n, p)$ \\
\hline $\mathrm{Na}-24$ & ENDFb 6 & $\mathrm{Mg}-24$ & $(n, p)$ \\
\hline $\mathrm{Na}-24$ & ENDFb5/532dos & $\mathrm{Al}-27$ & $(n, a)$ \\
\hline $\mathrm{Na}-24$ & LLNL/ACTL & $\mathrm{Al}-27$ & $(n, a)$ \\
\hline $\mathrm{Na}-24$ & ENDFb 6 & $\mathrm{Al}-27$ & $(n, a)$ \\
\hline $\mathrm{Na}-25$ & LLNL/ACTL & $\mathrm{Mg}-25$ & $(n, p)$ \\
\hline $\mathrm{Na}-26$ & LLNL/ACTL & $\mathrm{Mg}-26$ & $(n, p)$ \\
\hline $\mathrm{Na}-26$ & ENDFb 6 & $\mathrm{Al}-27$ & $(n, 2 p)$ \\
\hline$M g-23$ & LLNL/ACTL & $\mathrm{Mg}-24$ & $(n, 2 n)$ \\
\hline $\mathrm{Mg}-27$ & LLNL/ACTL & $\mathrm{Mg}-26$ & $(n, g)$ \\
\hline $\mathrm{Mg}-27$ & ENDFb5/532dos & $\mathrm{Al}-27$ & $(n, p)$ \\
\hline$M g-27$ & LLNL/ACTL & Al-27 & $(\mathrm{n}, \mathrm{p})$ \\
\hline $\mathrm{Mg}-27$ & ENDFb 6 & Al-27 & $(n, p)$ \\
\hline$M g-27$ & LLNL/ACTL & $\mathrm{Si}-30$ & $(n, a)$ \\
\hline $\mathrm{Mg}-27$ & ENDFb 6 & $\mathrm{Si}-30$ & $(n, a)$ \\
\hline$A 1-26$ & LLNL/ACTL & $A 1-27$ & $(n, 2 n)$ \\
\hline$A 1-26$ & ENDL 94 & $\mathrm{Al}-27$ & $(n, 2 n)$ \\
\hline Al-26 & ENDFb 6 & $A 1-27$ & $(n, 2 n)$ \\
\hline Al -28 & LLNL/ACTL & $\mathrm{Si}-29$ & $(n, n p)$ \\
\hline Al-28 & ENDFb 6 & Si-29 & $(n, n p)$ \\
\hline Al-28 & ENDFb5/532dos & $A 1-27$ & $(n, g)$ \\
\hline Al -28 & LLNL/ACTL & $A 1-27$ & $(n, g)$ \\
\hline Al -28 & ENDL 94 & Al-27 & $(n, g)$ \\
\hline Al -28 & ENDFb 6 & Al-27 & $(n, g)$ \\
\hline Al -28 & LLNL/ACTL & $\mathrm{Si}-28$ & $(n, p)$ \\
\hline Al-28 & ENDFb 6 & $\mathrm{Si}-28$ & $(n, p)$ \\
\hline Al-28 & LLNL/ACTL & $P-31$ & $(n, a)$ \\
\hline$A 1-28$ & ENDFb 6 & $\mathrm{P}-31$ & $(n, a)$ \\
\hline Al-29 & LLNL/ACTL & $\mathrm{Si}-30$ & $(n, n p)$ \\
\hline Al-29 & ENDFb 6 & $\mathrm{Si}-30$ & $(n, n p)$ \\
\hline Al-29 & LLNL/ACTL & $\mathrm{Si}-29$ & $(n, p)$ \\
\hline Al-29 & ENDFb 6 & $\mathrm{Si}-29$ & $(n, p)$ \\
\hline Al-30 & LLNL/ACTL & $\mathrm{Si}-30$ & $(n, p)$ \\
\hline Al-30 & ENDFb 6 & $\mathrm{Si}-30$ & $(n, p)$ \\
\hline Si-27 & LLNL/ACTL & $\mathrm{Si}-28$ & $(n, 2 n)$ \\
\hline Si-27 & ENDFb 6 & $\mathrm{Si}-28$ & $(n, 2 n)$ \\
\hline $\mathrm{Si}-31$ & LLNL/ACTL & $\mathrm{Si}-30$ & $(n, g)$ \\
\hline $\mathrm{Si}-31$ & ENDFb 6 & $\mathrm{Si}-30$ & $(n, g)$ \\
\hline $\mathrm{Si}-31$ & ENDFb5/532dos & $P-3 I$ & $(n, p)$ \\
\hline $\mathrm{Si}-31$ & LLNL/ACTL & $\mathrm{P}-31$ & $(n, p)$ \\
\hline $\mathrm{Si}-31$ & ENDFb 6 & $P-31$ & $(n, p)$ \\
\hline
\end{tabular}


UCRL-TR-203529

\begin{tabular}{|c|c|c|c|c|c|c|c|}
\hline Si-31 & LLNL/ACTL & $S-34$ & $(n, a)$ & $K-43$ & LLNL/ACTL & $\mathrm{Ca}-43$ & $(n, p)$ \\
\hline Si-32 & LLNL/ACTL & $s-36$ & $(\mathrm{n}, \mathrm{na})$ & $\mathrm{K}-43$ & LLNL/ACTL & $\mathrm{Ca}-44$ & $(n, d)$ \\
\hline$P-30$ & LLNL/ACTL & $P-31$ & $(n, 2 n)$ & $\mathrm{K}-43$ & ENDFb 6 & $S C-45$ & $(\mathrm{n}, 3 \mathrm{He}$ \\
\hline$P-30$ & ENDL 94 & $P-31$ & $(n, 2 n)$ & $K-44$ & LLNL/ACTL & $\mathrm{Ca}-44$ & $(n, p)$ \\
\hline$P-30$ & ENDFb 6 & $P-31$ & $(n, 2 n)$ & $K-46$ & LLNL/ACTL & $\mathrm{Ca}-46$ & $(n, p)$ \\
\hline$P-30$ & LLNL/ACTL & $s-32$ & $(n, t)$ & $K-48$ & LLNL/ACTL & $\mathrm{Ca}-48$ & $(n, p)$ \\
\hline$P-30$ & ENDFb 6 & $s-32$ & $(n, t)$ & $\mathrm{Ca}-39$ & LLNL/ACTL & $\mathrm{Ca}-40$ & $(n, 2 n)$ \\
\hline$P-32$ & LLNL/ACTL & $s-33$ & $(n, n p)$ & $\mathrm{Ca}-41$ & LLNL/ACTL & $\mathrm{Ca}-42$ & $(n, 2 n)$ \\
\hline$P-32$ & LLNL/ACTL & $P-31$ & $(n, g)$ & $\mathrm{Ca}-41$ & LLNL/ACTL & $\mathrm{Ca}-40$ & $(n, g)$ \\
\hline$P-32$ & ENDL94 & $P-31$ & $(n, g)$ & $\mathrm{Ca}-45$ & LLNL/ACTL & $\mathrm{Ca}-46$ & $(n, 2 n)$ \\
\hline$P-32$ & ENDFb 6 & $P-31$ & $(n, g)$ & $\mathrm{Ca}-45$ & LLNL/ACTL & Ti-49 & $(\mathrm{n}, \mathrm{na})$ \\
\hline$P-32$ & ENDFb5/532dos & $S-.32$ & $(\mathrm{n}, \mathrm{p})$ & $\mathrm{Ca}-45$ & LLNL/ACTL & $\mathrm{Ca}-44$ & $(n, g)$ \\
\hline$P-32$ & LLNL/ACTL & $s-32$ & $(n, p)$ & $\mathrm{Ca}-45$ & LLNL/ACTL & $S C-45$ & $(n, p)$ \\
\hline$P-32$ & ENDFb 6 & $s-32$ & $(n, p)$ & $\mathrm{Ca}-45$ & ENDFb 6 & $S c-45$ & $(n, p)$ \\
\hline$P-32$ & LLNL/ACTL & $S-33$ & $(n, d)$ & $\mathrm{Ca}-45$ & ENDFb5/532dos & $\mathrm{Ti}-48$ & $(n, a)$ \\
\hline$P-32$ & LLNL/ACTL & $\mathrm{Cl}-35$ & $(n, a)$ & $\mathrm{Ca}-45$ & LLNL/ACTL & $\mathrm{Ti}-48$ & $(n, a)$ \\
\hline$P-33$ & LLNL/ACTL & $\mathrm{Cl}-37$ & $(n, n a)$ & $\mathrm{Ca}-45$ & ENDFb 6 & $\mathrm{Ti}-48$ & $(n, a)$ \\
\hline$P-33$ & LLNL/ACTL & $S-34$ & $(n, n p)$ & $\mathrm{Ca}-47$ & LLNL/ACTL & $\mathrm{Ca}-48$ & $(n, 2 n)$ \\
\hline$P-33$ & LLNL/ACTL & $s-33$ & $(\mathrm{n}, \mathrm{p})$ & $\mathrm{Ca}-47$ & LLNL/ACTL & $\mathrm{Ca}-46$ & $(n, g)$ \\
\hline$P-33$ & LLNL/ACTL & $S-34$ & $(n, d)$ & $\mathrm{Ca}-47$ & ENDFb5/532dos & $\mathrm{Ti}-50$ & $(n, a)$ \\
\hline$P-34$ & LLNL/ACTL & $S-34$ & $(n, p)$ & $\mathrm{Ca}-47$ & LLNL/ACTL & $\mathrm{Ti}-50$ & $(n, a)$ \\
\hline$P-34$ & LLNL/ACTL & $\mathrm{Cl}-37$ & $(n, a)$ & $\mathrm{Ca}-47$ & ENDFb 6 & $\mathrm{Ti}-50$ & $(n, a)$ \\
\hline$S-31$ & LLNL/ACTL & $S-32$ & $(n, 2 n)$ & $\mathrm{Ca}-49$ & LLNL/ACTL & $\mathrm{Ca}-48$ & $(n, g)$ \\
\hline$S-31$ & ENDL 94 & $S-32$ & $(n, 2 n)$ & $S c-44$ & ENDFb5/532dos & $S C-45$ & $(n, 2 n)$ \\
\hline$S-31$ & ENDFb 6 & $S-32$ & $(n, 2 n)$ & $S c-44$ & LLNL/ACTL & $S C-45$ & $(n, 2 n)$ \\
\hline$S-35$ & LLNL/ACTL & $s-36$ & $(n, 2 n)$ & $S C-44$ & ENDFb 6 & $S C-45$ & $(n, 2 n)$ \\
\hline$S-35$ & LLNL/ACTL & $S-34$ & $(n, g)$ & $S c-44$ & LLNL/ACTL & $\mathrm{Ti}-46$ & $(n, t)$ \\
\hline$S-35$ & LLNL/ACTL & $\mathrm{Cl}-35$ & $(\mathrm{n}, \mathrm{p})$ & $S c-46$ & LLNL/ACTL & $V-50$ & $(\mathrm{n}, \mathrm{na})$ \\
\hline$S-35$ & LLNL/ACTL & $\mathrm{Cl}-37$ & $(n, t)$ & $S c-46$ & ENDFb5/532dos & Ti-47 & $(n, n p)$ \\
\hline$S-35$ & LLNL/ACTL & Ar -38 & $(n, a)$ & $S C-46$ & LLNL/ACTL & $\mathrm{Ti}-47$ & $(\mathrm{n}, \mathrm{np})$ \\
\hline$S-37$ & LLNL/ACTL & $s-36$ & $(n, g)$ & $S C-46$ & ENDFb 6 & $\mathrm{Ti}-47$ & $(\mathrm{n}, \mathrm{np})$ \\
\hline$S-37$ & LLNL/ACTL & $\mathrm{Cl}-37$ & $(\mathrm{n}, \mathrm{p})$ & $S c-46$ & ENDFb5/532dos & $S C-45$ & $(n, g)$ \\
\hline$S-37$ & LLNL/ACTL & Ar -40 & $(n, a)$ & $S c-46$ & LLNL/ACTL & $S C-45$ & $(n, g)$ \\
\hline $\mathrm{Cl}-34$ & LLNL/ACTL & $\mathrm{Cl}-35$ & $(n, 2 n)$ & $S c-46$ & ENDFb 6 & $S C-45$ & $(n, g)$ \\
\hline $\mathrm{Cl}-36$ & LLNL/ACTL & $\mathrm{Cl}-37$ & $(n, 2 n)$ & $S c-46$ & ENDFb5/532dos & $\mathrm{Ti}-46$ & $(n, p)$ \\
\hline $\mathrm{Cl}-36$ & LLNL/ACTL & $\mathrm{Cl}-35$ & $(n, g)$ & $S c-46$ & LLNL/ACTL & $\mathrm{Ti}-46$ & $(n, p)$ \\
\hline $\mathrm{Cl}-36$ & LLNL/ACTL & Ar-36 & $(n, p)$ & $S C-46$ & ENDFb 6 & $\mathrm{Ti}-46$ & $(\mathrm{n}, \mathrm{p})$ \\
\hline $\mathrm{Cl}-36$ & LLNL/ACTL & $\mathrm{K}-39$ & $(n, a)$ & $S c-46$ & LLNL/ACTL & $\mathrm{Ti}-47$ & $(n, d)$ \\
\hline $\mathrm{Cl}-38$ & LLNL/ACTL & $\mathrm{Cl}-37$ & $(n, g)$ & $S c-47$ & LLNL/ACTL & $V-51$ & $(\mathrm{n}, \mathrm{na})$ \\
\hline $\mathrm{Cl}-38$ & LLNL/ACTL & Ar-38 & $(n, p)$ & $S C-47$ & ENDL94 & $V-51$ & $(n, n a)$ \\
\hline $\mathrm{Cl}-38$ & LLNL/ACTL & $\mathrm{K}-41$ & $(\mathrm{n}, \mathrm{a})$ & $S C-47$ & ENDFb5/532dos & $\mathrm{Ti}-48$ & $(\mathrm{n}, \mathrm{np})$ \\
\hline $\mathrm{Cl}-40$ & LLNL/ACTL & Ar -40 & $(n, p)$ & $S C-47$ & LLNL/ACTL & $\mathrm{Ti}-48$ & $(n, n p)$ \\
\hline Ar -36 & LLNL/ACTL & Ar-36 & $(n, 2 n)$ & $S C-47$ & ENDFb 6 & $\mathrm{Ti}-48$ & $(\mathrm{n}, \mathrm{np})$ \\
\hline Ar -37 & LLNL/ACTL & Ar -38 & $(n, 2 n)$ & $S c-47$ & ENDFb5/532dos & $\mathrm{Ti}-47$ & $(n, p)$ \\
\hline Ar -37 & LLNL/ACTL & Ar-36 & $(n, g)$ & $S C-47$ & LLNL/ACTL & $\mathrm{Ti}-47$ & $(n, p)$ \\
\hline Ar -37 & LLNL/ACTL & $\mathrm{K}-39$ & $(n, t)$ & $S c-47$ & ENDFb 6 & $\mathrm{Ti}-47$ & $(n, p)$ \\
\hline Ar -37 & LLNL/ACTL & $\mathrm{Ca}-40$ & $(n, a)$ & $S c-47$ & LLNL/ACTL & $\mathrm{Ti}-48$ & $(n, d)$ \\
\hline Ar-39 & LLNL/ACTL & Ar -40 & $(n, 2 n)$ & $S c-47$ & LLNL/ACTL & $V-50$ & $(n, a)$ \\
\hline Ar-39 & LLNL/ACTL & $\mathrm{Ca}-43$ & $(n, n a)$ & $S c-48$ & LLNL/ACTL & Ti-49 & $(n, n p)$ \\
\hline Ar-39 & LLNL/ACTL & Ar-38 & $(n, g)$ & $S c-48$ & ENDFb5/532dos & $\mathrm{Ti}-48$ & $(n, p)$ \\
\hline Ar-39 & LLNL/ACTL & $\mathrm{K}-39$ & $(n, p)$ & $S c-48$ & LLNL/ACTL & $\mathrm{Ti}-48$ & $(n, p)$ \\
\hline Ar-39 & LLNL/ACTL & $\mathrm{K}-40$ & $(n, d)$ & $S c-48$ & ENDFb 6 & $\mathrm{Ti}-48$ & $(n, p)$ \\
\hline Ar-39 & LLNL/ACTL & $\mathrm{K}-41$ & $(n, t)$ & $S c-48$ & LLNL/ACTL & Ti-49 & $(n, d)$ \\
\hline Ar-39 & LLNL/ACTL & $\mathrm{Ca}-42$ & $(n, a)$ & $S c-48$ & LLNL/ACTL & $V-51$ & $(n, a)$ \\
\hline Ar -41 & ENDFb5/532dos & Ar-40 & $(n, g)$ & $S C-49$ & LLNL/ACTL & $\mathrm{Ti}-50$ & $(\mathrm{n}, \mathrm{np})$ \\
\hline Ar -41 & LLNL/ACTL & Ar -40 & $(n, g)$ & $S c-49$ & LLNL/ACTL & Ti-49 & $(\mathrm{n}, \mathrm{p})$ \\
\hline Ar -41 & ENDFb 6 & Ar-40 & $(n, g)$ & $S C-50$ & LLNL/ACTL & $\mathrm{Ti}-50$ & $(n, p)$ \\
\hline Ar -41 & ENDFb5/532dos & $\mathrm{K}-4 \mathrm{I}$ & $(n, p)$ & $\mathrm{Ti}-45$ & LLNL/ACTL & $\mathrm{Ti}-46$ & $(n, 2 n)$ \\
\hline Ar -41 & LLNL/ACTL & $K-41$ & $(\mathrm{n}, \mathrm{p})$ & $\mathrm{Ti}-51$ & LLNL/ACTL & $\mathrm{Ti}-50$ & $(n, g)$ \\
\hline Ar -41 & ENDFb 6 & $\mathrm{~K}-41$ & $(\mathrm{n}, \mathrm{p})$ & $\mathrm{Ti}-51$ & LLNL/ACTL & $V-51$ & $(n, p)$ \\
\hline Ar -41 & LLNL/ACTL & $\mathrm{Ca}-44$ & $(n, a)$ & $\mathrm{Ti}-51$ & LLNL/ACTL & $\operatorname{Cr}-54$ & $(n, a)$ \\
\hline Ar -43 & LLNL/ACTL & $\mathrm{Ca}-46$ & $(n, a)$ & Ti-51 & ENDFb 6 & Cr -54 & $(n, a)$ \\
\hline$K-38$ & LLNL/ACTL & $\mathrm{K}-39$ & $(n, 2 n)$ & $V-48$ & LLNL/ACTL & $\operatorname{Cr}-50$ & $(n, t)$ \\
\hline $\mathrm{K}-38$ & LLNL/ACTL & $\mathrm{Ca}-40$ & $(n, t)$ & $V-49$ & LLNL/ACTL & $V-50$ & $(n, 2 n)$ \\
\hline$K-40$ & LLNL/ACTL & $K-41$ & $(n, 2 n)$ & $V-49$ & LLNL/ACTL & $\operatorname{Cr}-50$ & $(n, n p)$ \\
\hline$K-40$ & LLNL/ACTL & $K-39$ & $(n, g)$ & $V-49$ & ENDFb 6 & $\mathrm{Cr}-50$ & $(n, n p)$ \\
\hline $\mathrm{K}-40$ & LLNL/ACTL & $\mathrm{Ca}-40$ & $(n, p)$ & $V-49$ & LLNL/ACTL & $\operatorname{Cr}-50$ & $(n, d)$ \\
\hline$K-42$ & LLNL/ACTL & $K-41$ & $(n, g)$ & $V-49$ & ENDFb 6 & $\operatorname{Cr}-50$ & $(n, d)$ \\
\hline$K-42$ & LLNL/ACTL & $\mathrm{Ca}-42$ & $(n, p)$ & $V-50$ & LLNL/ACTL & $V-51$ & $(n, 2 n)$ \\
\hline$K-42$ & LLNL/ACTL & $\mathrm{Ca}-43$ & $(n, d)$ & $V-50$ & ENDL94 & $V-51$ & $(n, 2 n)$ \\
\hline$K-42$ & LLNL/ACTL & $S c-45$ & $(n, a)$ & $V-50$ & LLNL/ACTL & $\operatorname{Cr}-50$ & $(n, p)$ \\
\hline$K-42$ & ENDFb 6 & $S C-45$ & $(n, a)$ & $V-50$ & ENDFb 6 & $\operatorname{Cr}-50$ & $(n, p)$ \\
\hline
\end{tabular}


UCRL-TR-203529

\begin{tabular}{|c|c|c|c|c|c|c|c|}
\hline$V-50$ & LLNL/ACTL & $\mathrm{Cr}-52$ & $(n, t)$ & $\mathrm{Fe}-55$ & LLNL/ACTL & $\mathrm{Fe}-57$ & $(n, 3 n)$ \\
\hline$V-52$ & LLNL/ACTL & $\mathrm{Cr}-53$ & $(n, n p)$ & $\mathrm{Fe}-55$ & LLNL/ACTL & $\mathrm{Fe}-54$ & $(n, g)$ \\
\hline$V-52$ & ENDFb 6 & $\mathrm{Cr}-53$ & $(n, n p)$ & $\mathrm{Fe}-55$ & ENDFb 6 & $\mathrm{Fe}-54$ & $(n, g)$ \\
\hline$V-52$ & LLNL/ACTL & $V-51$ & $(n, g)$ & $\mathrm{Fe}-55$ & ENDFb5/532dos & $\mathrm{Ni}-58$ & $(n, a)$ \\
\hline$V-52$ & ENDL 94 & $V-51$ & $(n, g)$ & $\mathrm{Fe}-55$ & LLNL/ACTL & $\mathrm{Ni}-58$ & $(n, a)$ \\
\hline$V-52$ & LLNL/ACTL & $\mathrm{Cr}-52$ & $(n, p)$ & $\mathrm{Fe}-55$ & ENDFb 6 & $\mathrm{Ni}-58$ & $(n, a)$ \\
\hline$V-52$ & ENDFb 6 & $\mathrm{Cr}-52$ & $(n, p)$ & $\mathrm{Fe}-59$ & LLNL/ACTL & $\mathrm{Fe}-58$ & $(n, g)$ \\
\hline$V-52$ & LLNL/ACTL & $\mathrm{Cr}-53$ & $(n, d)$ & $\mathrm{Fe}-59$ & ENDFb 6 & $\mathrm{Fe}-58$ & $(n, g)$ \\
\hline$V-52$ & LLNL/ACTL & $\mathrm{Cr}-54$ & $(n, t)$ & $\mathrm{Fe}-59$ & LLNL/ACTL & $\mathrm{Co}-59$ & $(n, p)$ \\
\hline$V-52$ & LLNL/ACTL & $M n-55$ & $(n, a)$ & $\mathrm{Fe}-59$ & ENDFb 6 & $\mathrm{Co}-59$ & $(n, p)$ \\
\hline$V-52$ & ENDFb 6 & $M n-55$ & $(n, a)$ & $\mathrm{Fe}-59$ & ENDFb5/532dos & $\mathrm{Ni}-62$ & $(n, a)$ \\
\hline$V-52$ & ENDFb 6 & $\mathrm{Co}-59$ & $(n, 2 a)$ & $\mathrm{Fe}-59$ & LLNL/ACTL & $\mathrm{Ni}-62$ & $(n, a)$ \\
\hline$V-53$ & LLNL/ACTL & $\mathrm{Cr}-54$ & $(n, n p)$ & $\mathrm{Fe}-59$ & ENDFb 6 & $\mathrm{Ni}-62$ & $(n, a)$ \\
\hline$V-53$ & LLNL/ACTL & Cr -53 & $(n, p)$ & $\mathrm{Fe}-60$ & LLNL/ACTL & $\mathrm{Ni}-64$ & $(\mathrm{n}, \mathrm{na})$ \\
\hline$V-53$ & ENDFb 6 & $\mathrm{Cr}-53$ & $(n, p)$ & $\mathrm{Fe}-60$ & ENDFb 6 & $\mathrm{Ni}-64$ & $(\mathrm{n}, \mathrm{na})$ \\
\hline$V-53$ & LLNL/ACTL & Cr -54 & $(n, d)$ & $\mathrm{Fe}-60$ & ENDFb 6 & $\mathrm{Ni}-61$ & $(n, 2 p)$ \\
\hline$V-53$ & ENDFb 6 & $M n-55$ & $(\mathrm{n}, 3 \mathrm{He})$ & $\mathrm{Fe}-61$ & LLNL/ACTL & $\mathrm{Ni}-64$ & $(n, a)$ \\
\hline$V-54$ & LLNL/ACTL & $\mathrm{Cr}-54$ & $(\mathrm{n}, \mathrm{p})$ & $\mathrm{Fe}-61$ & ENDFb 6 & $\mathrm{Ni}-64$ & $(n, a)$ \\
\hline$V-54$ & ENDFb 6 & $\mathrm{Cr}-54$ & $(n, p)$ & $\mathrm{Co}-56$ & LLNL/ACTL & $\mathrm{Ni}-58$ & $(n, t)$ \\
\hline $\mathrm{Cr}-49$ & ENDFb5/532dos & $\mathrm{Cr}-50$ & $(n, 2 n)$ & $\mathrm{Co}-57$ & LLNL/ACTL & $\mathrm{Ni}-58$ & $(\mathrm{n}, \mathrm{np})$ \\
\hline $\mathrm{Cr}-49$ & LLNL/ACTL & $\mathrm{Cr}-50$ & $(n, 2 n)$ & $\mathrm{Co}-57$ & ENDL 94 & $\mathrm{Ni}-58$ & $(\mathrm{n}, \mathrm{np})$ \\
\hline $\mathrm{Cr}-49$ & ENDFb 6 & $\mathrm{Cr}-50$ & $(n, 2 n)$ & $\mathrm{Co}-57$ & ENDFb 6 & $\mathrm{Ni}-58$ & $(\mathrm{n}, \mathrm{np})$ \\
\hline Cr-51 & ENDFb5/532dos & $\mathrm{Cr}-52$ & $(n, 2 n)$ & $\mathrm{Co}-57$ & ENDFb5/532dos & $\mathrm{Ni}-58$ & $(n, d)$ \\
\hline $\mathrm{Cr}-51$ & LLNL/ACTL & $\mathrm{Cr}-52$ & $(n, 2 n)$ & $\mathrm{Co}-57$ & LLNL/ACTL & $\mathrm{Ni}-58$ & $(n, d)$ \\
\hline Cr-51 & ENDFb 6 & $\mathrm{Cr}-52$ & $(n, 2 n)$ & $\mathrm{Co}-57$ & ENDFb 6 & $\mathrm{Ni}-58$ & $(n, d)$ \\
\hline $\mathrm{Cr}-51$ & ENDFb5/532dos & $\mathrm{Cr}-50$ & $(n, g)$ & $\mathrm{Co}-58$ & LLNL/ACTL & $\mathrm{Co}-59$ & $(n, 2 n)$ \\
\hline $\mathrm{Cr}-51$ & LLNL/ACTL & $\mathrm{Cr}-50$ & $(n, g)$ & $\mathrm{Co}-58$ & ENDL 94 & $\mathrm{Co}-59$ & $(n, 2 n)$ \\
\hline Cr-51 & ENDFb 6 & $\mathrm{Cr}-50$ & $(n, g)$ & $\mathrm{Co}-58$ & ENDFb 6 & $\mathrm{Co}-59$ & $(n, 2 n)$ \\
\hline $\mathrm{Cr}-51$ & LLNL/ACTL & $\mathrm{Fe}-54$ & $(n, a)$ & Co-59 & ENDFb5/532dos & $\mathrm{Ni}-58$ & $(n, p)$ \\
\hline Cr-51 & ENDFb 6 & $\mathrm{Fe}-54$ & $(n, a)$ & $\mathrm{Co}-58$ & LLNL/ACTL & $\mathrm{Ni}-58$ & $(n, p)$ \\
\hline $\mathrm{Fe}-55$ & ENDFb5/532dos & $\mathrm{Fe}-54$ & $(n, g)$ & $\mathrm{Co}-58$ & ENDFb 6 & $\mathrm{Ni}-58$ & $(n, p)$ \\
\hline $\mathrm{Cr}-55$ & LLNL/ACTL & $\mathrm{Cr}-54$ & $(n, g)$ & $\mathrm{Co}-58$ & LLNL/ACTL & $\mathrm{Ni}-60$ & $(n, t)$ \\
\hline $\mathrm{Cr}-55$ & ENDFb 6 & $\mathrm{Cr}-54$ & $(n, g)$ & Co- 60 & LLNL/ACTL & $\mathrm{Ni}-61$ & $(\mathrm{n}, \mathrm{np})$ \\
\hline $\mathrm{Cr}-55$ & LLNL/ACTL & $M n-55$ & $(n, p)$ & $\mathrm{Co}-60$ & ENDFb 6 & $\mathrm{Ni}-61$ & $(\mathrm{n}, \mathrm{np})$ \\
\hline Cr-55 & ENDFb 6 & $M n-55$ & $(n, p)$ & $\mathrm{Co}-60$ & LLNL/ACTL & $\mathrm{Co}-59$ & $(n, g)$ \\
\hline $\mathrm{Cr}-55$ & LLNL/ACTL & $\mathrm{Fe}-58$ & $(n, a)$ & $\mathrm{Co}-60$ & ENDL 94 & $\mathrm{Co}-59$ & $(n, g)$ \\
\hline $\mathrm{Cr}-55$ & ENDFb 6 & $\mathrm{Fe}-58$ & $(n, a)$ & $\mathrm{Co}-60$ & ENDFb 6 & $\mathrm{Co}-59$ & $(n, g)$ \\
\hline Cr -55 & ENDFb 6 & $\mathrm{Co}-59$ & $(\mathrm{n}, \mathrm{pa})$ & $\mathrm{Co}-60$ & ENDFb5/532dos & $\mathrm{Ni}-60$ & $(n, p)$ \\
\hline$M n-52$ & LLNL/ACTL & $\mathrm{Fe}-54$ & $(n, t)$ & $\mathrm{Co}-60$ & LLNL/ACTL & $\mathrm{Ni}-60$ & $(n, p)$ \\
\hline$M n-53$ & LLNL/ACTL & $\mathrm{Fe}-54$ & $(n, n p)$ & $\mathrm{Co}-60$ & ENDFb 6 & $\mathrm{Ni}-60$ & $(n, p)$ \\
\hline$M n-53$ & ENDFb 6 & $\mathrm{Fe}-54$ & $(n, n p)$ & $\mathrm{Co}-60$ & LLNL/ACTL & $\mathrm{Ni}-61$ & $(n, d)$ \\
\hline$M n-53$ & LLNL/ACTL & $\mathrm{Fe}-54$ & $(n, d)$ & $\mathrm{Co}-60$ & LLNL/ACTL & $\mathrm{Ni}-62$ & $(n, t)$ \\
\hline$M n-53$ & ENDFb 6 & $\mathrm{Fe}-54$ & $(n, d)$ & $\mathrm{Co}-60$ & ENDFb5/532dos & $\mathrm{Cu}-63$ & $(n, a)$ \\
\hline$M n-54$ & LLNL/ACTL & $M n-55$ & $(n, 2 n)$ & $\mathrm{Co}-60$ & LLNL/ACTL & $\mathrm{Cu}-63$ & $(n, a)$ \\
\hline$M n-54$ & ENDL94 & $M n-55$ & $(n, 2 n)$ & $\mathrm{Co}-60$ & ENDFb 6 & $\mathrm{Cu}-63$ & $(n, a)$ \\
\hline$M n-54$ & ENDFb 6 & $M n-55$ & $(n, 2 n)$ & Co- 61 & LLNL/ACTL & $\mathrm{Cu}-65$ & $(\mathrm{n}, \mathrm{na})$ \\
\hline$M n-54$ & LLNL/ACTL & $\mathrm{Fe}-54$ & $(n, p)$ & $\mathrm{Co}-61$ & ENDFb 6 & $\mathrm{Cu}-65$ & $(\mathrm{n}, \mathrm{na})$ \\
\hline$M n-54$ & ENDFb 6 & $\mathrm{Fe}-54$ & $(n, p)$ & Co-61 & LLNL/ACTL & $\mathrm{Ni}-62$ & $(n, n p)$ \\
\hline$M n-54$ & LLNL/ACTL & $\mathrm{Fe}-56$ & $(n, t)$ & Co-61 & ENDFb 6 & $\mathrm{Ni}-62$ & $(n, n p)$ \\
\hline$M n-54$ & ENDFb 6 & $\mathrm{Fe}-56$ & $(n, t)$ & $\mathrm{Co}-61$ & LLNL/ACTL & $\mathrm{Ni}-61$ & $(n, p)$ \\
\hline$M n-56$ & LLNL/ACTL & $\mathrm{Fe}-57$ & $(n, n p)$ & $\mathrm{Co}-61$ & ENDFb 6 & $\mathrm{Ni}-61$ & $(n, p)$ \\
\hline$M n-56$ & ENDFb 6 & $\mathrm{Fe}-57$ & $(n, n p)$ & Co-61 & LLNL/ACTL & $\mathrm{Ni}-62$ & $(n, d)$ \\
\hline$M n-56$ & LLNL/ACTL & $M n-55$ & $(n, g)$ & Co-61 & ENDFb 6 & $\mathrm{Ni}-62$ & $(n, d)$ \\
\hline$M n-56$ & ENDL94 & $M n-55$ & $(n, g)$ & Co- 61 & ENDFb 6 & $\mathrm{Cu}-63$ & $(\mathrm{n}, 3 \mathrm{He})$ \\
\hline$M n-56$ & ENDFb 6 & $M n-55$ & $(n, g)$ & $\mathrm{Co}-62$ & LLNL/ACTL & $\mathrm{Ni}-62$ & $(n, p)$ \\
\hline$M n-56$ & LLNL/ACTL & $\mathrm{Fe}-56$ & $(n, p)$ & $\mathrm{Co}-62$ & ENDFb 6 & $\mathrm{Ni}-62$ & $(n, p)$ \\
\hline$M n-56$ & ENDFb 6 & $\mathrm{Fe}-56$ & $(n, p)$ & $\mathrm{Co}-62$ & LLNL/ACTL & $\mathrm{Ni}-64$ & $(n, t)$ \\
\hline$M n-56$ & LLNL/ACTL & $\mathrm{Fe}-58$ & $(n, t)$ & $\mathrm{Co}-62$ & LLNL/ACTL & $\mathrm{Cu}-65$ & $(n, a)$ \\
\hline$M n-56$ & LLNL/ACTL & $\mathrm{Co}-59$ & $(n, a)$ & $\mathrm{Co}-62$ & ENDFb 6 & $\mathrm{Cu}-65$ & $(n, a)$ \\
\hline$M n-56$ & ENDFb 6 & $\mathrm{Co}-59$ & $(n, a)$ & $\mathrm{Co}-63$ & LLNL/ACTL & $\mathrm{Ni}-64$ & $(n, n p)$ \\
\hline$M n-57$ & LLNL/ACTL & $\mathrm{Fe}-58$ & $(n, n p)$ & $\mathrm{Co}-63$ & ENDFb 6 & $\mathrm{Ni}-64$ & $(n, n p)$ \\
\hline$M n-57$ & ENDFb 6 & $\mathrm{Fe}-58$ & $(n, n p)$ & $\mathrm{Co}-63$ & LLNL/ACTL & $\mathrm{Ni}-64$ & $(n, d)$ \\
\hline$M n-57$ & LLNL/ACTL & $\mathrm{Fe}-57$ & $(n, p)$ & Co- 63 & ENDFb 6 & $\mathrm{Ni}-64$ & $(n, d)$ \\
\hline$M n-57$ & ENDFb 6 & $\mathrm{Fe}-57$ & $(n, p)$ & Co- 63 & ENDFb 6 & $\mathrm{Cu}-65$ & $(\mathrm{n}, 3 \mathrm{He})$ \\
\hline$M n-57$ & ENDFb 6 & $\mathrm{Co}-59$ & $(\mathrm{n}, 3 \mathrm{He})$ & Co- 64 & LLNL/ACTL & $\mathrm{Ni}-64$ & $(n, p)$ \\
\hline$M n-58$ & LLNL/ACTL & $\mathrm{Fe}-58$ & $(n, p)$ & Co- 64 & ENDFb 6 & $\mathrm{Ni}-64$ & $(\mathrm{n}, \mathrm{p})$ \\
\hline$M n-58$ & ENDFb 6 & $\mathrm{Fe}-58$ & $(n, p)$ & $\mathrm{Ni}-57$ & ENDFb5/532dos & $\mathrm{Ni}-58$ & $(n, 2 n)$ \\
\hline$M n-58$ & ENDFb 6 & $\mathrm{Co}-59$ & $(n, 2 p)$ & $\mathrm{Ni}-57$ & LLNL/ACTL & $\mathrm{Ni}-58$ & $(n, 2 n)$ \\
\hline $\mathrm{Fe}-53$ & LLNL/ACTL & $\mathrm{Fe}-54$ & $(n, 2 n)$ & $\mathrm{Ni}-57$ & ENDL 94 & $\mathrm{Ni}-58$ & $(n, 2 n)$ \\
\hline $\mathrm{Fe}-53$ & ENDFb 6 & $\mathrm{Fe}-54$ & $(n, 2 n)$ & $\mathrm{Ni}-57$ & ENDFb 6 & $\mathrm{Ni}-58$ & $(n, 2 n)$ \\
\hline $\mathrm{Fe}-55$ & LLNL/ACTL & $\mathrm{Fe}-56$ & $(n, 2 n)$ & $\mathrm{Ni}-59$ & ENDFb5/532dos & $\mathrm{Ni}-60$ & $(n, 2 n)$ \\
\hline $\mathrm{Fe}-55$ & ENDFb 6 & $\mathrm{Fe}-56$ & $(n, 2 n)$ & $\mathrm{Ni}-59$ & LLNL/ACTL & $\mathrm{Ni}-60$ & $(n, 2 n)$ \\
\hline
\end{tabular}


UCRL-TR-203529

\begin{tabular}{|c|c|c|c|c|c|c|c|}
\hline $\mathrm{Ni}-59$ & ENDFb 6 & $\mathrm{Ni}-60$ & $(n, 2 n)$ & As -73 & ENDL 94 & As -75 & $(n, 3 n)$ \\
\hline $\mathrm{Ni}-55$ & ENDFb5/532dos & $\mathrm{Ni}-58$ & $(n, g)$ & As -74 & LLNL/ACTL & As -75 & $(n, 2 n)$ \\
\hline $\mathrm{Ni}-59$ & LLNL/ACTL & $\mathrm{Ni}-58$ & $(n, g)$ & As -74 & ENDL94 & As -75 & $(n, 2 n)$ \\
\hline $\mathrm{Ni}-59$ & ENDFb 6 & $\mathrm{Ni}-58$ & $(n, g)$ & As -76 & LLNL/ACTL & As -75 & $(n, g)$ \\
\hline $\mathrm{Ni}-63$ & LLNL/ACTL & $\mathrm{Ni}-64$ & $(n, 2 n)$ & As -76 & ENDL94 & As -75 & $(n, g)$ \\
\hline $\mathrm{Ni}-63$ & ENDFb 6 & $\mathrm{Ni}-64$ & $(n, 2 n)$ & As -76 & ENDFb 6 & As -75 & $(n, g)$ \\
\hline $\mathrm{Ni}-63$ & LLNL/ACTL & $\mathrm{Zn}-67$ & $(n, n a)$ & As -76 & LLNL/ACTL & $\mathrm{Br}-79$ & $(n, a)$ \\
\hline $\mathrm{Ni}-63$ & ENDFb5/532dos & $\mathrm{Ni}-62$ & $(n, g)$ & As -77 & LLNL/ACTL & $\mathrm{Br}-81$ & $(\mathrm{n}, \mathrm{na})$ \\
\hline $\mathrm{Ni}-63$ & LLNL/ACTL & $\mathrm{Ni}-62$ & $(n, g)$ & $\mathrm{Se}-73$ & LLNL/ACTL & $S e-74$ & $(n, 2 n)$ \\
\hline $\mathrm{Ni}-63$ & ENDFb 6 & $\mathrm{Ni}-62$ & $(n, g)$ & $\mathrm{Se}-75$ & LLNL/ACTL & $\mathrm{Se}-76$ & $(n, 2 n)$ \\
\hline $\mathrm{Ni}-62$ & ENDFb5/532dos & $\mathrm{Cu}-63$ & $(n, p)$ & $\mathrm{Se}-75$ & ENDFb 6 & $\mathrm{Se}-74$ & $(n, g)$ \\
\hline $\mathrm{Ni}-63$ & LLNL/ACTL & $\mathrm{Cu}-63$ & $(n, p)$ & $\mathrm{Se}-75$ & ENDFb 6 & $\mathrm{Kr}-78$ & $(n, a)$ \\
\hline $\mathrm{Ni}-63$ & ENDFb 6 & $\mathrm{Cu}-63$ & $(n, p)$ & $\mathrm{Se}-79$ & LLNL/ACTL & $\mathrm{Se}-80$ & $(n, 2 n)$ \\
\hline $\mathrm{Ni}-63$ & ENDFb 6 & $\mathrm{Cu}-65$ & $(n, t)$ & $\mathrm{Se}-79$ & ENDFb 6 & $\mathrm{Se}-78$ & $(n, g)$ \\
\hline $\mathrm{Ni}-63$ & LLNL/ACTL & $\mathrm{Zn}-66$ & $(n, a)$ & $\mathrm{Se}-79$ & LLNL/ACTL & $\mathrm{Br}-79$ & $(n, p)$ \\
\hline $\mathrm{Ni}-65$ & LLNL/ACTL & $\mathrm{Ni}-64$ & $(n, g)$ & $\mathrm{Se}-79$ & ENDFb 6 & $\mathrm{Kr}-82$ & $(n, a)$ \\
\hline $\mathrm{Ni}-65$ & ENDFb 6 & $\mathrm{Ni}-64$ & $(n, g)$ & $\mathrm{Se}-81$ & LLNL/ACTL & $\mathrm{Se}-82$ & $(n, 2 n)$ \\
\hline $\mathrm{Ni}-65$ & LLNL/ACTL & $\mathrm{Cu}-65$ & $(n, p)$ & $\mathrm{Se}-81$ & ENDFb 6 & $\mathrm{Se}-80$ & $(n, g)$ \\
\hline $\mathrm{Ni}-65$ & ENDFb 6 & $\mathrm{Cu}-65$ & $(n, p)$ & $\mathrm{Se}-81$ & ENDFb 6 & $\mathrm{Kr}-83$ & $(\mathrm{n}, 3 \mathrm{He})$ \\
\hline $\mathrm{Ni}-65$ & LLNL/ACTL & $\mathrm{Zn}-68$ & $(n, a)$ & $\mathrm{Se}-81$ & ENDFb 6 & $\mathrm{Kr}-84$ & $(n, a)$ \\
\hline $\mathrm{Ni}-66$ & LLNL/ACTL & $\mathrm{Zn}-70$ & $(n, n a)$ & $\mathrm{Se}-82$ & ENDFb 6 & $\mathrm{Kr}-84$ & $(\mathrm{n}, 3 \mathrm{He})$ \\
\hline $\mathrm{Ni}-67$ & LLNL/ACTL & $\mathrm{Zn}-70$ & $(n, a)$ & $\mathrm{Se}-83$ & ENDFb 6 & $\mathrm{Se}-82$ & $(n, g)$ \\
\hline $\mathrm{Cu}-62$ & $\mathrm{LLNL} / \mathrm{ACTL}$ & $\mathrm{Cu}-63$ & $(n, 2 n)$ & $\mathrm{Br}-76$ & ENDFb 6 & $\mathrm{Kr}-78$ & $(n, t)$ \\
\hline $\mathrm{Cu}-62$ & ENDFb 6 & $\mathrm{Cu}-63$ & $(n, 2 n)$ & $\mathrm{Br}-77$ & ENDFb 6 & $\mathrm{Kr}-78$ & $(n, d)$ \\
\hline $\mathrm{Cu}-64$ & ENDFb5/532dos & $\mathrm{Cu}-65$ & $(n, 2 n)$ & $\mathrm{Br}-78$ & LLNL/ACTL & $\mathrm{Br}-79$ & $(n, 2 n)$ \\
\hline $\mathrm{Cu}-64$ & LLNL/ACTL & $\mathrm{Cu}-65$ & $(n, 2 n)$ & $\mathrm{Br}-78$ & ENDFb 6 & $\mathrm{Kr}-78$ & $(n, p)$ \\
\hline $\mathrm{Cu}-64$ & ENDFb 6 & $\mathrm{Cu}-65$ & $(n, 2 n)$ & $\mathrm{Br}-78$ & ENDFb 6 & $\mathrm{Kr}-80$ & $(n, t)$ \\
\hline $\mathrm{Cu}-64$ & ENDFb5/532dos & $\mathrm{Cu}-63$ & $(n, g)$ & $\mathrm{Br}-80$ & LLNL/ACTL & $\mathrm{Br}-81$ & $(n, 2 n)$ \\
\hline $\mathrm{Cu}-64$ & LLNL/ACTL & $\mathrm{Cu}-63$ & $(n, g)$ & $\mathrm{Br}-80$ & ENDFb 6 & $\mathrm{Br}-79$ & $(n, g)$ \\
\hline $\mathrm{Cu}-64$ & ENDFb 6 & $\mathrm{Cu}-63$ & $(n, g)$ & $\mathrm{Br}-80$ & ENDFb 6 & $\mathrm{Kr}-80$ & $(n, p)$ \\
\hline $\mathrm{Cu}-64$ & LLNL/ACTL & $\mathrm{Zn}-64$ & $(n, p)$ & $\mathrm{Br}-80$ & ENDFb 6 & $\mathrm{Kr}-82$ & $(n, t)$ \\
\hline $\mathrm{Cu}-66$ & LLNL/ACTL & $\mathrm{Zn}-67$ & $(n, n p)$ & $\mathrm{Br}-82$ & LLNL/ACTL & $\mathrm{Br}-81$ & $(n, g)$ \\
\hline $\mathrm{Cu}-66$ & LLNL/ACTL & $\mathrm{Cu}-65$ & $(n, g)$ & $\mathrm{Br}-82$ & ENDFb 6 & $\mathrm{Br}-81$ & $(n, g)$ \\
\hline $\mathrm{Cu}-66$ & ENDFb 6 & $\mathrm{Cu}-65$ & $(n, g)$ & $\mathrm{Br}-82$ & ENDFb 6 & $\mathrm{Kr}-82$ & $(n, p)$ \\
\hline $\mathrm{Cu}-66$ & LLNL/ACTL & $\mathrm{Zn}-66$ & $(n, p)$ & $\mathrm{Br}-82$ & ENDFb 6 & $\mathrm{Kr}-83$ & $(n, d)$ \\
\hline $\mathrm{Cu}-67$ & LLNL/ACTL & $\mathrm{Zn}-68$ & $(n, n p)$ & $\mathrm{Br}-82$ & ENDFb 6 & $\mathrm{Kr}-84$ & $(n, t)$ \\
\hline $\mathrm{Cu}-67$ & LLNL/ACTL & $\mathrm{Zn}-67$ & $(n, p)$ & $\mathrm{Br}-82$ & ENDFb 6 & $\mathrm{Rb}-85$ & $(n, a)$ \\
\hline $\mathrm{Cu}-68$ & LLNL/ACTL & $\mathrm{Zn}-68$ & $(n, p)$ & $\mathrm{Br}-83$ & ENDFb 6 & $\mathrm{Rb}-87$ & $(n, n a)$ \\
\hline $\mathrm{Cu}-70$ & LLNL/ACTL & $\mathrm{Zn}-70$ & $(n, p)$ & $\mathrm{Br}-83$ & ENDFb 6 & $\mathrm{Kr}-83$ & $(n, p)$ \\
\hline$Z n-63$ & LLNL/ACTL & $\mathrm{Zn}-64$ & $(n, 2 n)$ & $\mathrm{Br}-83$ & ENDFb 6 & $\mathrm{Kr}-84$ & $(n, d)$ \\
\hline$Z n-65$ & LLNL/ACTL & $2 n-66$ & $(n, 2 n)$ & $\mathrm{Br}-83$ & ENDFb 6 & $\mathrm{Rb}-85$ & $(\mathrm{n}, 3 \mathrm{He})$ \\
\hline$Z n-65$ & LLNL/ACTL & $\mathrm{Zn}-64$ & $(n, g)$ & $\mathrm{Br}-84$ & ENDFb 6 & $\mathrm{Kr}-84$ & $(n, p)$ \\
\hline $\mathrm{Zn}-79$ & LLNL/ACTL & $\mathrm{Zn}-70$ & $(n, 2 n)$ & $\mathrm{Br}-84$ & ENDFb 6 & $K r-86$ & $(n, t)$ \\
\hline$Z n-69$ & LLNL/ACTL & $\mathrm{Ge}-73$ & $(n, n a)$ & $\mathrm{Br}-84$ & ENDFb 6 & $\mathrm{Rb}-87$ & $(n, a)$ \\
\hline$Z n-69$ & LLNL/ACTL & $\mathrm{Zn}-68$ & $(n, g)$ & $\mathrm{Br}-85$ & ENDFb 6 & $K r-86$ & $(n, d)$ \\
\hline$Z n-69$ & LLNL/ACTL & $\mathrm{Ge}-72$ & $(n, a)$ & $\mathrm{Br}-85$ & ENDFb 6 & $\mathrm{Rb}-87$ & $(\mathrm{n}, 3 \mathrm{He})$ \\
\hline $\mathrm{Zn}-71$ & LLNL/ACTL & $\mathrm{Zn}-70$ & $(n, g)$ & $\mathrm{Br}-86$ & ENDFb 6 & $\mathrm{Kr}-86$ & $(n, p)$ \\
\hline $\mathrm{Zn}-71$ & LLNL/ACTL & $\mathrm{Ge}-74$ & $(n, a)$ & $\mathrm{Kr}-77$ & ENDFb 6 & $\mathrm{Kr}-78$ & $(n, 2 n)$ \\
\hline $\mathrm{Zn}-72$ & LLNL/ACTL & $\mathrm{Ge}-76$ & $(n, n a)$ & $\mathrm{Kr}-79$ & ENDFb 6 & $\mathrm{Kr}-80$ & $(n, 2 n)$ \\
\hline $\mathrm{Zn}-73$ & LLNL/ACTL & $\mathrm{Ge}-76$ & $(n, a)$ & $\mathrm{Kr}-79$ & ENDFb 6 & $\mathrm{Kr}-78$ & $(n, g)$ \\
\hline $\mathrm{Ga}-68$ & LLNL/ACTL & $\mathrm{Ga}-69$ & $(n, 2 n)$ & $\mathrm{Kr}-81$ & ENDFb 6 & $\mathrm{Kr}-82$ & $(n, 2 n)$ \\
\hline $\mathrm{Ga}-70$ & LLNL/ACTL & $\mathrm{Ga}-71$ & $(n, 2 n)$ & $\mathrm{Kr}-81$ & ENDFb 6 & $\mathrm{Kr}-83$ & $(n, 3 n)$ \\
\hline $\mathrm{Ga}-70$ & LLNL/ACTL & $\mathrm{Ge}-70$ & $(n, p)$ & $\mathrm{Kr}-81$ & ENDFb 6 & $\mathrm{Kr}-80$ & $(n, g)$ \\
\hline $\mathrm{Ga}-72$ & LLNL/ACTL & $\mathrm{Ge}-73$ & $(n, n p)$ & $K r-85$ & ENDFb 6 & $\mathrm{Kr}-86$ & $(n, 2 n)$ \\
\hline $\mathrm{Ga}-72$ & LLNL/ACTL & $\mathrm{Ga}-71$ & $(n, g)$ & $K r-85$ & ENDFb 6 & $\mathrm{Kr}-84$ & $(n, g)$ \\
\hline $\mathrm{Ga}-72$ & LLNL/ACTL & $\mathrm{Ge}-72$ & $(n, p)$ & $\mathrm{Kr}-85$ & ENDFb 6 & $\mathrm{Rb}-85$ & $(n, p)$ \\
\hline $\mathrm{Ga}-72$ & LLNL/ACTL & As -75 & $(n, a)$ & $\mathrm{Kr}-85$ & ENDFb 6 & $\mathrm{Rb}-87$ & $(n, t)$ \\
\hline $\mathrm{Ga}-73$ & LLNL/ACTL & $\mathrm{Ge}-74$ & $(n, n p)$ & $\mathrm{Kr}-87$ & ENDFb 6 & $\mathrm{Kr}-86$ & $(n, g)$ \\
\hline $\mathrm{Ga}-73$ & LLNL/ACTL & $\mathrm{Ge}-73$ & $(n, p)$ & $\mathrm{Kr}-87$ & ENDFb 6 & $\mathrm{Rb}-87$ & $(n, p)$ \\
\hline $\mathrm{Ga}-74$ & LLNL/ACTL & $\mathrm{Ge}-74$ & $(n, p)$ & $R b-84$ & LLNL/ACTL & $\mathrm{Rb}-85$ & $(n, 2 n)$ \\
\hline $\mathrm{Ga}-75$ & LLNL/ACTL & $\mathrm{Ge}-76$ & $(n, n p)$ & $R b-84$ & ENDFb 6 & $\mathrm{Rb}-85$ & $(n, 2 n)$ \\
\hline $\mathrm{Ga}-76$ & LLNL/ACTL & $\mathrm{Ge}-76$ & $(n, p)$ & $\mathrm{Rb}-86$ & LLNL/ACTL & $\mathrm{Rb}-87$ & $(n, 2 n)$ \\
\hline $\mathrm{Ge}-69$ & LLNL/ACTL & $\mathrm{Ge}-70$ & $(n, 2 n)$ & $\mathrm{Rb}-86$ & ENDFb 6 & $\mathrm{Rb}-87$ & $(n, 2 n)$ \\
\hline $\mathrm{Ge}-71$ & LLNL/ACTL & $\mathrm{Ge}-72$ & $(n, 2 n)$ & $\mathrm{Rb}-86$ & ENDFb 6 & $\mathrm{Rb}-85$ & $(n, g)$ \\
\hline $\mathrm{Ge}-71$ & LLNL/ACTL & $\mathrm{Ge}-73$ & $(n, 3 n)$ & $\mathrm{Rb}-88$ & ENDFb 6 & $\mathrm{Rb}-87$ & $(n, g)$ \\
\hline $\mathrm{Ge}-71$ & LLNL/ACTL & $\mathrm{Ge}-70$ & $(n, g)$ & $S r-83$ & LLNL/ACTL & $\mathrm{Sr}-84$ & $(n, 2 n)$ \\
\hline $\mathrm{Ge}-75$ & LLNL/ACTL & $\mathrm{Ge}-76$ & $(n, 2 n)$ & $S r-85$ & ENDFb 6 & $S r-84$ & $(n, g)$ \\
\hline $\mathrm{Ge}-75$ & LLNL/ACTL & $\mathrm{Ge}-74$ & $(n, g)$ & Sr-89 & ENDFb 6 & $\mathrm{Sr}-88$ & $(n, g)$ \\
\hline $\mathrm{Ge}-75$ & ENDFb 6 & $\mathrm{Ge}-74$ & $(n, g)$ & Sr-89 & LLNL/ACTL & $\mathrm{Zr}-92$ & $(n, a)$ \\
\hline $\mathrm{Ge}-75$ & LLNL/ACTL & As -75 & $(n, p)$ & Sr-89 & ENDFb 6 & Zr-92 & $(n, a)$ \\
\hline $\mathrm{Ge}-77$ & LLNL/ACTL & $\mathrm{Ge}-76$ & $(n, g)$ & Sr-90 & LLNL/ACTL & Zr-94 & $(n, n a)$ \\
\hline $\mathrm{Ge}-77$ & ENDFb 6 & $\mathrm{Ge}-76$ & $(n, g)$ & Sr-91 & LLNL/ACTL & Zr-94 & $(n, a)$ \\
\hline
\end{tabular}


UCRL-TR-203529

\begin{tabular}{|c|c|c|c|c|c|c|c|}
\hline Sr -91 & ENDFb 6 & Zr-94 & $(n, a)$ & Mo-101 & ENDFb5/532dos & $\mathrm{Md}-\mathrm{I} 00$ & $(n, g)$ \\
\hline $\operatorname{Sr}-93$ & ENDFb 6 & Zr-96 & $(n, a)$ & Mo-101 & LLNL/ACTL & Mo-100 & $(n, g)$ \\
\hline$Y-90$ & LLNL/ACTL & Zr-91 & $(n, n p)$ & Mo-101 & ENDFb 6 & Mo-100 & $(n, g)$ \\
\hline$Y-90$ & LLNL/ACTL & Zr-90 & $(n, p)$ & $\mathrm{Ru}-97$ & ENDFb 6 & $\mathrm{Pd}-102$ & $(n, 2 n a)$ \\
\hline$Y-90$ & ENDFb 6 & Zr-90 & $(n, p)$ & $\mathrm{Ru}-97$ & ENDFb 6 & $\mathrm{Ru}-96$ & $(n, g)$ \\
\hline$Y-90$ & LLNL/ACTL & Zr-91 & $(n, d)$ & $\mathrm{Ru}-103$ & ENDFb 6 & $\mathrm{Pd}-108$ & $(n, 2 n a)$ \\
\hline$Y-90$ & LLNL/ACTL & $\mathrm{Nb}-93$ & $(n, a)$ & $\mathrm{Ru}-103$ & ENDFb 6 & $\mathrm{Ru}-102$ & $(n, g)$ \\
\hline$Y-90$ & ENDFb 6 & $\mathrm{Nb}-93$ & $(n, a)$ & $\mathrm{Ru}-103$ & ENDFb 6 & $P d-106$ & $(n, a)$ \\
\hline$Y-91$ & LLNL/ACTL & Zr-92 & $(\mathrm{n}, \mathrm{np})$ & $\mathrm{Ru}-105$ & ENDFb 6 & $\mathrm{Pd}-110$ & $(\mathrm{n}, 2 \mathrm{na})$ \\
\hline$Y-91$ & ENDFb 6 & $Y-90$ & $(n, g)$ & $\mathrm{Ru}-105$ & ENDFb 6 & $\mathrm{Ru}-104$ & $(n, g)$ \\
\hline$Y-91$ & LLNL/ACTL & Zr-91 & $(n, p)$ & $\mathrm{Ru}-105$ & ENDFb 6 & $\mathrm{Pd}-108$ & $(n, a)$ \\
\hline$Y-91$ & ENDFb 6 & Zr-91 & $(n, p)$ & $\mathrm{Ru}-106$ & ENDFb 6 & $P d-110$ & $(\mathrm{n}, \mathrm{na})$ \\
\hline$Y-91$ & LLNL/ACTL & Zr-92 & $(n, d)$ & $\mathrm{Ru}-107$ & ENDFb 6 & $\mathrm{Pd}-110$ & $(n, a)$ \\
\hline$Y-92$ & LLNL/ACTL & Zr-92 & $(n, p)$ & $\mathrm{Rh}-100$ & ENDFb 6 & $P d-102$ & $(\mathrm{n}, \mathrm{nd})$ \\
\hline$Y-92$ & ENDFb 6 & Zr-92 & $(n, p)$ & $\mathrm{Rh}-100$ & ENDFb 6 & $\mathrm{Pd}-102$ & $(n, 2 n p)$ \\
\hline$Y-94$ & LLNL/ACTL & Zr-94 & $(n, p)$ & $\mathrm{Rh}-101$ & ENDFb 6 & $\mathrm{Pd}-102$ & $(n, n p)$ \\
\hline$Y-94$ & ENDFb 6 & Zr-94 & $(n, p)$ & $\mathrm{Rh}-101$ & ENDFb 6 & $\mathrm{Pd}-102$ & $(n, d)$ \\
\hline Zr-E9 & ENDFb5/532dos & Zr-90 & $(n, 2 n)$ & $\mathrm{Rh}-102$ & LLNL/ACTL & $\mathrm{Rh}-103$ & $(n, 2 n)$ \\
\hline $\mathrm{Zr}-89$ & LLNL/ACTL & Zr-90 & $(n, 2 n)$ & $\mathrm{Rh}-102$ & ENDFb 6 & $\mathrm{Rh}-103$ & $(n, 2 n)$ \\
\hline Zr-89 & ENDFb 6 & Zr-90 & $(n, 2 n)$ & $\mathrm{Rh}-102$ & ENDFb 6 & $\mathrm{Pd}-104$ & $(n, n d)$ \\
\hline Zr-89 & LLNL/ACTL & Zr-91 & $(n, 3 n)$ & $\mathrm{Rh}-102$ & ENDFb 6 & $P d-104$ & $(n, 2 n p)$ \\
\hline Zr-89 & LLNL/ACTL & Mo-92 & $(n, a)$ & $\mathrm{Rh}-102$ & ENDFb 6 & $\mathrm{Pd}-102$ & $(n, p)$ \\
\hline Zr-93 & $\mathrm{LLNL} / \mathrm{ACTL}$ & Zr-94 & $(n, 2 n)$ & $\mathrm{Rh}-104$ & ENDFb 6 & $P d-105$ & $(\mathrm{n}, \mathrm{np})$ \\
\hline Zr-93 & ENDFb 6 & Zr-94 & $(n, 2 n)$ & Rh-104 & ENDFb 6 & $P d-106$ & $(\mathrm{n}, \mathrm{nd})$ \\
\hline Zr-93 & ENDFb5/532dos & Zr-92 & $(n, g)$ & $\mathrm{Rh}-104$ & ENDFb 6 & $P d-106$ & $(n, 2 n p)$ \\
\hline Zr-93 & LLNL/ACTL & Zr-92 & $(n, g)$ & $\mathrm{Rh}-104$ & ENDFb 6 & $\mathrm{Rh}-103$ & $(n, g)$ \\
\hline Zr-93 & ENDFb 6 & Zr-92 & $(n, g)$ & $\mathrm{Rh}-104$ & ENDFb 6 & $\mathrm{Pd}-104$ & $(n, p)$ \\
\hline Zr-93 & LLNL/ACTL & $\mathrm{Nb}-93$ & $(n, p)$ & $\mathrm{Rh}-104$ & ENDFb 6 & $\mathrm{Pd}-105$ & $(n, d)$ \\
\hline Zr-93 & ENDFb 6 & $\mathrm{Nb}-93$ & $(n, p)$ & $\mathrm{Rh}-104$ & LLNL/ACTL & $\mathrm{Ag}-107$ & $(n, a)$ \\
\hline $\mathrm{Zr}-93$ & LLNL/ACTL & Mo-96 & $(n, a)$ & Rh-104 & ENDFb 6 & $\mathrm{Ag}-107$ & $(n, a)$ \\
\hline Zr-95 & LLNL/ACTL & Zr-96 & $(n, 2 n)$ & $\mathrm{Rh}-105$ & ENDFb 6 & $\mathrm{Ag}-109$ & $(\mathrm{n}, \mathrm{na})$ \\
\hline Zr-95 & ENDFb 6 & Zr-96 & $(n, 2 n)$ & $\mathrm{Rh}-105$ & ENDFb 6 & $P d-106$ & $(n, n p)$ \\
\hline Zr-95 & ENDFb5/532dos & Zr-94 & $(n, g)$ & $R h-105$ & ENDFb 6 & $P d-105$ & $(\mathrm{n}, \mathrm{p})$ \\
\hline Zr-95 & LLNL/ACTL & Zr-94 & $(n, g)$ & $\mathrm{Rh}-105$ & ENDFb 6 & $P d-106$ & $(n, d)$ \\
\hline Zr-95 & ENDFb 6 & Zr-94 & $(n, g)$ & $\mathrm{Rh}-105$ & ENDFb 6 & $\mathrm{Ag}-107$ & $(\mathrm{n}, 3 \mathrm{He})$ \\
\hline Zr-95 & LLNL/ACTL & Mo-98 & $(n, a)$ & $\mathrm{Rh}-106$ & ENDFb 6 & $P d-108$ & $(\mathrm{n}, \mathrm{nd})$ \\
\hline Zr-97 & ENDFb 6 & Zr-96 & $(n, g)$ & $\mathrm{Rh}-106$ & ENDFb 6 & $P d-108$ & $(n, 2 n p)$ \\
\hline Zr-97 & LLNL/ACTL & Mo-100 & $(n, a)$ & $\mathrm{Rh}-106$ & ENDFb 6 & $P d-106$ & $(n, p)$ \\
\hline $\mathrm{Nb}-90$ & LLNL/ACTL & Mo-92 & $(n, t)$ & $\mathrm{Rh}-106$ & LLNL/ACTL & $\mathrm{Ag}-109$ & $(n, a)$ \\
\hline $\mathrm{Nb}-91$ & LLNL/ACTL & $\mathrm{Nb}-93$ & $(n, 3 n)$ & $\mathrm{Rh}-106$ & ENDFb 6 & $\mathrm{Ag}-109$ & $(n, a)$ \\
\hline $\mathrm{Nb}-91$ & ENDL 94 & $\mathrm{Nb}-93$ & $(n, 3 n)$ & $\mathrm{Rh}-107$ & ENDFb 6 & $\mathrm{Pd}-108$ & $(\mathrm{n}, \mathrm{np})$ \\
\hline $\mathrm{Nb}-91$ & ENDFb 6 & $\mathrm{Nb}-93$ & $(n, 3 n)$ & $\mathrm{Rh}-107$ & ENDFb 6 & $\mathrm{Pd}-108$ & $(n, d)$ \\
\hline $\mathrm{Nb}-91$ & LLNL/ACTL & Mo-92 & $(n, n p)$ & Rh-107 & ENDFb 6 & $\mathrm{Ag}-109$ & $(\mathrm{n}, 3 \mathrm{He})$ \\
\hline $\mathrm{Nb}-91$ & LLNL/ACTL & Mo-92 & $(n, d)$ & $\mathrm{Rh}-108$ & ENDFb 6 & $\mathrm{Pd}-110$ & $(n, n d)$ \\
\hline $\mathrm{Nb}-92$ & LLNL/ACTL & $\mathrm{Nb}-93$ & $(n, 2 n)$ & Rh-108 & ENDFb 6 & $\mathrm{Pd}-110$ & $(n, 2 n p)$ \\
\hline $\mathrm{Nb}-92$ & ENDL94 & $\mathrm{Nb}-93$ & $(n, 2 n)$ & $\mathrm{Rh}-108$ & ENDFb 6 & $\mathrm{Pd}-108$ & $(n, p)$ \\
\hline $\mathrm{Nb}-92$ & ENDFb 6 & $\mathrm{Nb}-93$ & $(n, 2 n)$ & Rh-109 & ENDFb 6 & $\mathrm{Pd}-110$ & $(\mathrm{n}, \mathrm{np})$ \\
\hline $\mathrm{Nb}-92$ & LLNL/ACTL & Mo-92 & $(n, p)$ & Rh-109 & ENDFb 6 & $\mathrm{Pd}-110$ & $(n, d)$ \\
\hline $\mathrm{Nb}-92$ & LLNL/ACTL & Mo-94 & $(n, t)$ & $\mathrm{Rh}-110$ & ENDFb 6 & $\mathrm{Pd}-110$ & $(n, p)$ \\
\hline $\mathrm{Nb}-94$ & LLNL/ACTL & Mo-95 & $(n, n p)$ & $P d-100$ & ENDFb 6 & $P d-102$ & $(n, 3 n)$ \\
\hline $\mathrm{Nb}-94$ & LLNL/ACTL & $\mathrm{Nb}-93$ & $(n, g)$ & $P d-101$ & ENDFb 6 & $\mathrm{Pd}-102$ & $(n, 2 n)$ \\
\hline $\mathrm{Nb}-94$ & ENDL94 & $\mathrm{Nb}-93$ & $(n, g)$ & $P d-103$ & ENDFb 6 & $\mathrm{Pd}-104$ & $(n, 2 n)$ \\
\hline $\mathrm{Nb}-94$ & ENDFb 6 & $\mathrm{Nb}-93$ & $(n, g)$ & $P d-103$ & ENDFb 6 & $\mathrm{Pd}-105$ & $(n, 3 n)$ \\
\hline $\mathrm{Nb}-94$ & LLNL/ACTL & Mo-94 & $(n, p)$ & $P d-103$ & ENDFb 6 & $\mathrm{Pd}-102$ & $(n, g)$ \\
\hline $\mathrm{Nb}-94$ & LLNL/ACTL & Mo-95 & $(n, d)$ & Pd-103 & ENDFb 6 & $\mathrm{Cd}-106$ & $(n, a)$ \\
\hline $\mathrm{Nb}-95$ & LLNL/ACTL & Mo-96 & $(n, n p)$ & $P d-107$ & ENDFb 6 & $P d-108$ & $(n, 2 n)$ \\
\hline $\mathrm{Nb}-95$ & LLNL/ACTL & Mo-95 & $(n, p)$ & $P d-107$ & ENDFb 6 & $\mathrm{Cd}-111$ & $(\mathrm{n}, \mathrm{na})$ \\
\hline $\mathrm{Nb}-95$ & LLNL/ACTL & Mo-96 & $(n, d)$ & $P d-107$ & ENDFb 6 & $P d-106$ & $(n, g)$ \\
\hline $\mathrm{Nb}-96$ & LLNL/ACTL & Mo-96 & $(n, p)$ & $P d-107$ & LLNL/ACTL & $\mathrm{Ag}-107$ & $(n, p)$ \\
\hline $\mathrm{Nb}-97$ & LLNL/ACTL & Mo-97 & $(n, p)$ & $P d-107$ & ENDFb 6 & $\mathrm{Ag}-107$ & $(n, p)$ \\
\hline $\mathrm{Nb}-98$ & LLNL/ACTL & Mo-98 & $(\mathrm{n}, \mathrm{p})$ & $P d-107$ & ENDFb 6 & $\mathrm{Ag}-109$ & $(n, t)$ \\
\hline $\mathrm{Nb}-100$ & LLNL/ACTL & Mo-100 & $(n, p)$ & Pd-107 & ENDFb 6 & $\mathrm{Cd}-110$ & $(n, a)$ \\
\hline Mo-91 & LLNL/ACTL & Mo-92 & $(n, 2 n)$ & $P d-109$ & ENDFb 6 & $\mathrm{Pd}-110$ & $(n, 2 n)$ \\
\hline Mo-93 & LLNL/ACTL & Mo-94 & $(n, 2 n)$ & $P d-109$ & ENDFb 6 & $\mathrm{Cd}-113$ & $(n, n a)$ \\
\hline Mo-93 & LLNL/ACTL & Mo-95 & $(n, 3 n)$ & $P d-109$ & ENDFb 6 & $\mathrm{Pd}-108$ & $(n, g)$ \\
\hline Mo-93 & ENDFb5/532dos & Mo-92 & $(n, g)$ & $P d-109$ & LLNL/ACTL & $\mathrm{Ag}-109$ & $(n, p)$ \\
\hline Mo-93 & LLNL/ACTL & Mo-92 & $(n, g)$ & $P d-109$ & ENDFb 6 & $\mathrm{Ag}-109$ & $(n, p)$ \\
\hline Mo-93 & ENDFb 6 & Mo-92 & $(n, g)$ & Pd-109 & ENDFb 6 & $\mathrm{~cd}-111$ & $(\mathrm{n}, 3 \mathrm{He})$ \\
\hline Mo-99 & LLNL/ACTL & Mo-100 & $(n, 2 n)$ & $P d-109$ & LLNL/ACTL & $\mathrm{Cd}-112$ & $(n, a)$ \\
\hline Mo-99 & ENDFb5/532dos & Mo-99 & $(n, g)$ & $P d-109$ & ENDFb 6 & $\mathrm{Cd}-112$ & $(n, a)$ \\
\hline Mo-99 & LLNL/ACTL & Mo-98 & $(n, g)$ & $\mathrm{Pd}-111$ & LLNL/ACTL & $\mathrm{Pd}-110$ & $(n, g)$ \\
\hline Mo-99 & ENDFb 6 & Mo-98 & $(n, g)$ & $P d-111$ & ENDFb 6 & $P d-110$ & $(n, g)$ \\
\hline
\end{tabular}


UCRL-TR-203529

\begin{tabular}{|c|c|c|c|c|c|c|c|}
\hline $\mathrm{Pd}-111$ & ENDFb 6 & $\mathrm{Cd}-113$ & $(\mathrm{n}, 3 \mathrm{He})$ & $\mathrm{Cd}-113$ & ENDFb 6 & $\mathrm{Cd}-112$ & $(n, g)$ \\
\hline $\mathrm{Pd}-111$ & ENDFb 6 & $\mathrm{Cd}-114$ & $(n, a)$ & $\mathrm{Cd}-113$ & LLNL/ACTL & In-113 & $(n, p)$ \\
\hline Pd-112 & ENDFb 6 & $\mathrm{Cd}-116$ & $(n, n a)$ & $\mathrm{Cd}-115$ & LLNL/ACTL & $\mathrm{Cd}-116$ & $(n, 2 n)$ \\
\hline $\mathrm{Pd}-112$ & ENDFb 6 & $\mathrm{Cd}-114$ & $(\mathrm{n}, 3 \mathrm{He})$ & $\mathrm{Cd}-115$ & ENDFb 6 & $\mathrm{Cd}-116$ & $(n, 2 n)$ \\
\hline $\mathrm{Pd}-113$ & ENDFb 6 & $\mathrm{Cd}-116$ & $(n, a)$ & $\mathrm{Cd}-115$ & ENDFb 6 & $\mathrm{Cd}-114$ & $(n, g)$ \\
\hline $\mathrm{Pd}-114$ & ENDFb 6 & $\mathrm{~cd}-116$ & $(\mathrm{n}, 3 \mathrm{He})$ & $\mathrm{Cd}-115$ & LLNL/ACTL & In-115 & $(n, p)$ \\
\hline Ag-103 & ENDFb 6 & $\mathrm{Cd}-106$ & $(n, n t)$ & $\mathrm{Cd}-115$ & LLNL/ACTL & $\mathrm{Sn}-118$ & $(n, a)$ \\
\hline $\mathrm{Ag}-104$ & ENDFb 6 & $\mathrm{Cd}-106$ & $(n, n d)$ & $\mathrm{Cd}-117$ & ENDFb 6 & $\mathrm{Cd}-116$ & $(n, g)$ \\
\hline $\mathrm{Ag}-104$ & ENDFb 6 & $\mathrm{Cd}-106$ & $(n, t)$ & In-111 & LLNL/ACTL & $\mathrm{Sn}-112$ & $(n, n p)$ \\
\hline Ag-105 & LLNL/ACTL & $\mathrm{Ag}-107$ & $(n, 3 n)$ & In-111 & LLNL/ACTL & $\mathrm{Sn}-112$ & $(n, d)$ \\
\hline Ag-105 & ENDL 94 & $\mathrm{Ag}-107$ & $(n, 3 n)$ & In -112 & LLNL/ACTL & In-113 & $(n, 2 n)$ \\
\hline Ag-105 & ENDFb 6 & $\mathrm{Ag}-107$ & $(n, 3 n)$ & In -112 & LLNL/ACTL & $\mathrm{Sn}-112$ & $(n, p)$ \\
\hline Ag-105 & ENDFb 6 & $\mathrm{Cd}-106$ & $(n, n p)$ & In-114 & LLNL/ACTL & In-115 & $(n, 2 n)$ \\
\hline $\mathrm{Ag}-105$ & ENDFb 6 & $\mathrm{Cd}-108$ & $(n, n t)$ & In-114 & LLNL/ACTL & $\mathrm{Sn}-115$ & $(n, n p)$ \\
\hline Ag-105 & ENDFb 6 & $\mathrm{Cd}-106$ & $(n, d)$ & In-114 & LLNL/ACTL & In-113 & $(n, g)$ \\
\hline $\mathrm{Ag}-106$ & LLNL/ACTL & $\mathrm{Ag}-107$ & $(n, 2 n)$ & In-114 & ENDFb 6 & In -113 & $(n, g)$ \\
\hline $\mathrm{Ag}-106$ & ENDL 94 & $\mathrm{Ag}-107$ & $(n, 2 n)$ & In-114 & LLNL/ACTL & $\mathrm{Sn}-114$ & $(n, p)$ \\
\hline $\mathrm{Ag}-106$ & ENDFb 6 & $\mathrm{Ag}-107$ & $(n, 2 n)$ & In-115 & LLNL/ACTL & $\mathrm{Sn}-116$ & $(\mathrm{n}, \mathrm{np})$ \\
\hline $\mathrm{Ag}-106$ & ENDFb 6 & $\mathrm{Cd}-108$ & $(n, n d)$ & In -115 & ENDFb5/532dos & In-Ii5 & $\left(n, n^{\prime}\right)$, \\
\hline $\mathrm{Ag}-106$ & ENDFb 6 & $\mathrm{Cd}-106$ & $(n, p)$ & In-115 & LLNL/ACTL & $\mathrm{Sn}-115$ & $(n, p)$ \\
\hline Ag-106 & ENDFb 6 & $\mathrm{Cd}-108$ & $(n, t)$ & In-116 & LLNL/ACTL & In-115 & $(n, g)$ \\
\hline $\mathrm{Ag}-108$ & LLNL/ACTL & Ag-109 & $(n, 2 n)$ & In-116 & ENDFb 6 & In-115 & $(n, g)$ \\
\hline $\mathrm{Ag}-108$ & ENDL94 & $\mathrm{Ag}-109$ & $(n, 2 n)$ & In-116 & LLNL/ACTL & $\mathrm{Sn}-116$ & $(n, p)$ \\
\hline $\mathrm{Ag}-108$ & ENDFb 6 & Ag-109 & $(n, 2 n)$ & In-117 & LLNL/ACTL & $\mathrm{Sn}-117$ & $(n, p)$ \\
\hline $\mathrm{Ag}-108$ & ENDFb 6 & $\mathrm{Cd}-110$ & $(n, n d)$ & In -118 & LLNL/ACTL & $\mathrm{Sn}-118$ & $(n, p)$ \\
\hline $\mathrm{Ag}-108$ & ENDFb 6 & $\mathrm{Cd}-111$ & $(n, n t)$ & In-119 & LLNL/ACTL & $\mathrm{Sn}-119$ & $(n, p)$ \\
\hline $\mathrm{Ag}-108$ & LLNL/ACTL & $\mathrm{Ag}-107$ & $(n, g)$ & In -120 & LLNL/ACTL & $\mathrm{Sn}-120$ & $(n, p)$ \\
\hline $\mathrm{Ag}-108$ & ENDL 94 & $\mathrm{Ag}-107$ & $(n, g)$ & In -122 & LLNL/ACTL & $\mathrm{Sn}-122$ & $(n, p)$ \\
\hline Ag-108 & ENDFb 6 & $\mathrm{Ag}-107$ & $(n, g)$ & In-124 & LLNL/ACTL & $\mathrm{Sn}-124$ & $(n, p)$ \\
\hline $\mathrm{Ag}-108$ & ENDFb 6 & $\mathrm{Cd}-108$ & $(n, p)$ & $S n-111$ & LLNL/ACTL & $\mathrm{Sn}-112$ & $(n, 2 n)$ \\
\hline Ag-108 & ENDFb 6 & $\mathrm{Cd}-110$ & $(n, t)$ & $S n-113$ & LLNL/ACTL & $\mathrm{Sn}-114$ & $(n, 2 n)$ \\
\hline $\mathrm{Ag}-110$ & ENDFb 6 & $\mathrm{Cd}-111$ & $(n, n p)$ & $S n-113$ & LLNL/ACTL & $\mathrm{Sn}-112$ & $(n, g)$ \\
\hline $\mathrm{Ag}-110$ & ENDFb 6 & $\mathrm{Cd}-112$ & $(n, n d)$ & $S n-113$ & ENDFb 6 & $\mathrm{Sn}-112$ & $(n, g)$ \\
\hline $\mathrm{Ag}-110$ & ENDFb 6 & $\mathrm{Cd}-113$ & $(n, n t)$ & $\mathrm{Sn}-121$ & LLNL/ACTL & $\mathrm{Sn}-122$ & $(n, 2 n)$ \\
\hline $\mathrm{Ag}-110$ & LLNL/ACTL & Ag-109 & $(n, g)$ & $S n-121$ & ENDFb5/532dos & $\mathrm{Sn}-\mathrm{I} 20$ & $(n, g)$ \\
\hline Ag-110 & ENDL 94 & $\mathrm{Ag}-109$ & $(n, g)$ & $\mathrm{Sn}-121$ & LLNL/ACTL & $\mathrm{Sn}-120$ & $(n, g)$ \\
\hline Ag-110 & ENDFb 6 & $\mathrm{Ag}-109$ & $(n, g)$ & $\mathrm{Sn}-121$ & ENDFb 6 & $\mathrm{Sn}-120$ & $(n, g)$ \\
\hline $\mathrm{Ag}-110$ & ENDFb 6 & $\mathrm{Cd}-110$ & $(n, p)$ & $\mathrm{Sn}-121$ & LLNL/ACTL & $\mathrm{Sb}-121$ & $(n, p)$ \\
\hline $\mathrm{Ag}-110$ & ENDFb 6 & $\mathrm{~cd}-111$ & $(n, d)$ & $S n-123$ & LLNL/ACTL & $\mathrm{Sn}-124$ & $(n, 2 n)$ \\
\hline $\mathrm{Ag}-110$ & ENDFb 6 & $\mathrm{Cd}-112$ & $(n, t)$ & $\mathrm{Sn}-123$ & ENDFb5/532dos & $\mathrm{Sn}-\mathrm{I} 22$ & $(n, g)$ \\
\hline Ag-110 & LLNL/ACTL & In-113 & $(n, a)$ & Sn-123 & LLNL/ACTL & $\mathrm{Sn}-122$ & $(n, g)$ \\
\hline $\mathrm{Ag}-111$ & ENDFb 6 & $\mathrm{Cd}-112$ & $(n, n p)$ & $\mathrm{Sn}-123$ & ENDFb 6 & $\mathrm{Sn}-122$ & $(n, g)$ \\
\hline $\mathrm{Ag}-111$ & ENDFb 6 & $\mathrm{Cd}-113$ & $(\mathrm{n}, \mathrm{nd})$ & $\mathrm{Sn}-123$ & LLNL/ACTL & $\mathrm{Sb}-123$ & $(n, p)$ \\
\hline $\mathrm{Ag}-111$ & ENDFb 6 & $\mathrm{Cd}-114$ & $(n, n t)$ & $S n-125$ & ENDFb5/532dos & $\mathrm{Sn}-\mathrm{I} 24$ & $(n, g)$ \\
\hline Ag-111 & LLNL/ACTL & $\mathrm{Cd}-111$ & $(n, p)$ & $S n-125$ & LLNL/ACTL & $\mathrm{Sn}-124$ & $(n, g)$ \\
\hline $\mathrm{Ag}-111$ & ENDFb 6 & $\mathrm{Cd}-111$ & $(n, p)$ & $S n-125$ & ENDFb 6 & $\mathrm{Sn}-124$ & $(n, g)$ \\
\hline Ag-111 & ENDFb 6 & $\mathrm{Cd}-112$ & $(n, d)$ & $\mathrm{Sb}-120$ & LLNL/ACTL & $\mathrm{Sb}-121$ & $(n, 2 n)$ \\
\hline $\mathrm{Ag}-111$ & ENDFb 6 & $\mathrm{Cd}-113$ & $(n, t)$ & $\mathrm{Sb}-122$ & LLNL/ACTL & $\mathrm{Sb}-123$ & $(n, 2 n)$ \\
\hline $\mathrm{Ag}-112$ & ENDFb 6 & $\mathrm{Cd}-113$ & $(n, n p)$ & $\mathrm{Sb}-122$ & LLNL/ACTL & $\mathrm{Sb}-121$ & $(n, g)$ \\
\hline Ag-112 & ENDFb 6 & $\mathrm{Cd}-114$ & $(\mathrm{n}, \mathrm{nd})$ & $\mathrm{Sb}-122$ & ENDFb 6 & $\mathrm{Sb}-121$ & $(n, g)$ \\
\hline $\mathrm{Ag}-112$ & ENDFb 6 & $\mathrm{Cd}-112$ & $(n, p)$ & $S b-124$ & LLNL/ACTL & $\mathrm{Sb}-123$ & $(n, g)$ \\
\hline Ag-112 & ENDFb 6 & $\mathrm{Cd}-113$ & $(n, d)$ & $\mathrm{Sb}-124$ & ENDFb 6 & $\mathrm{Sb}-123$ & $(n, g)$ \\
\hline Ag-112 & ENDFb 6 & $\mathrm{Cd}-114$ & $(n, t)$ & $\mathrm{Sb}-124$ & ENDFb 6 & I-127 & $(n, a)$ \\
\hline $\mathrm{Ag}-109$ & LLNL/ACTL & In-115 & $(n, a)$ & $\mathrm{Te}-121$ & ENDFb 6 & $\mathrm{Te}-120$ & $(n, g)$ \\
\hline $\mathrm{Ag}-113$ & ENDFb 6 & $\mathrm{Cd}-114$ & $(n, n p)$ & $\mathrm{Te}-121$ & ENDFb 6 & $\mathrm{Xe}-124$ & $(n, a)$ \\
\hline $\mathrm{Ag}-113$ & ENDFb 6 & $\mathrm{~cd}-116$ & $(n, n t)$ & $\mathrm{Te}-123$ & ENDFb 6 & $\mathrm{Te}-122$ & $(n, g)$ \\
\hline Ag-113 & ENDFb 6 & $\mathrm{Cd}-113$ & $(n, p)$ & $\mathrm{Te}-123$ & ENDFb 6 & $\mathrm{Xe}-126$ & $(n, a)$ \\
\hline Ag-113 & ENDFb 6 & $\mathrm{Cd}-114$ & $(n, d)$ & $\mathrm{Te}-127$ & ENDFb 6 & $\mathrm{Te}-126$ & $(n, g)$ \\
\hline $\mathrm{Ag}-114$ & ENDFb 6 & $\mathrm{Cd}-116$ & $(n, n d)$ & $\mathrm{Te}-127$ & ENDFb 6 & $I-127$ & $(n, p)$ \\
\hline $\mathrm{Ag}-114$ & ENDFb 6 & $\mathrm{Cd}-114$ & $(n, p)$ & $\mathrm{Te}-127$ & ENDFb 6 & $\mathrm{Xe}-130$ & $(n, a)$ \\
\hline $\mathrm{Ag}-114$ & ENDFb 6 & $\mathrm{Cd}-116$ & $(n, t)$ & $\mathrm{Te}-128$ & ENDFb 6 & $\mathrm{Xe}-131$ & $(n, a)$ \\
\hline Ag-115 & ENDFb 6 & $\mathrm{~cd}-116$ & $(n, n p)$ & $\mathrm{Te}-129$ & ENDFb 6 & $\mathrm{Te}-128$ & $(n, g)$ \\
\hline Ag-115 & ENDFb 6 & $\mathrm{Cd}-116$ & $(n, d)$ & $\mathrm{Te}-129$ & ENDFb 6 & $\mathrm{Xe}-132$ & $(n, a)$ \\
\hline $\mathrm{Ag}-116$ & ENDFb 6 & $\mathrm{Cd}-116$ & $(n, p)$ & $\mathrm{Te}-131$ & ENDFb 6 & $\mathrm{Te}-130$ & $(n, g)$ \\
\hline $\mathrm{Cd}-105$ & LLNL/ACTL & $\mathrm{Cd}-106$ & $(n, 2 n)$ & $\mathrm{Te}-131$ & ENDFb 6 & $\mathrm{Xe}-134$ & $(n, a)$ \\
\hline $\mathrm{Cd}-105$ & ENDFb 6 & $\mathrm{Cd}-106$ & $(n, 2 n)$ & $\mathrm{Te}-133$ & ENDFb 6 & $\mathrm{Xe}-136$ & $(n, a)$ \\
\hline $\mathrm{Cd}-107$ & ENDFb 6 & $\mathrm{Cd}-108$ & $(n, 2 n)$ & $I-122$ & ENDFb 6 & $\mathrm{Xe}-124$ & $(n, t)$ \\
\hline $\mathrm{Cd}-107$ & ENDFb 6 & $\mathrm{Cd}-106$ & $(n, g)$ & $I-123$ & ENDFb 6 & $\mathrm{Xe}-124$ & $(n, d)$ \\
\hline $\mathrm{Cd}-109$ & ENDFb 6 & $\mathrm{Cd}-110$ & $(n, 2 n)$ & $I-124$ & ENDFb 6 & $\mathrm{Xe}-124$ & $(n, p)$ \\
\hline Cd-109 & ENDFb 6 & $\mathrm{Cd}-111$ & $(n, 3 n)$ & $I-124$ & ENDFb 6 & $\mathrm{Xe}-126$ & $(n, t)$ \\
\hline Cd-109 & ENDFb 6 & $\mathrm{Cd}-108$ & $(n, g)$ & $I-125$ & ENDL94 & I-127 & $(n, 3 n)$ \\
\hline $\mathrm{Cd}-113$ & ENDFb 6 & $\mathrm{Cd}-114$ & $(n, 2 n)$ & $I-125$ & ENDFb 6 & $I-127$ & $(n, 3 n)$ \\
\hline
\end{tabular}


UCRL-TR-203529

\begin{tabular}{|c|c|c|c|c|c|c|c|}
\hline$I-125$ & ENDFb 6 & $\mathrm{Xe}-126$ & $(n, d)$ & $\operatorname{Pr}-140$ & LLNL/ACTL & $\operatorname{Pr}-141$ & $(n, 2 n)$ \\
\hline$I-126$ & ENDFb5/532dos & $I-127$ & $(n, 2 n)$ & $\operatorname{Pr}-140$ & ENDFb 6 & $\operatorname{Pr}-141$ & $(n, 2 n)$ \\
\hline$I-126$ & LLNL/ACTL & $I-127$ & $(n, 2 n)$ & $\operatorname{Pr}-142$ & ENDFb 6 & $\mathrm{Nd}-143$ & $(n, n p)$ \\
\hline$I-126$ & ENDL94 & $I-127$ & $(n, 2 n)$ & $\operatorname{Pr}-142$ & ENDFb 6 & $\operatorname{Pr}-141$ & $(n, g)$ \\
\hline$I-126$ & ENDFb 6 & $I-127$ & $(n, 2 n)$ & $\operatorname{Pr}-142$ & ENDFb 6 & $\mathrm{Nd}-143$ & $(n, d)$ \\
\hline$I-126$ & ENDFb 6 & $\mathrm{Xe}-126$ & $(n, p)$ & $\operatorname{Pr}-143$ & ENDFb 6 & Pm-147 & $(\mathrm{n}, \mathrm{na})$ \\
\hline$I-126$ & ENDFb 6 & $\mathrm{Xe}-128$ & $(n, t)$ & $\operatorname{Pr}-143$ & ENDFb 6 & $\mathrm{Nd}-143$ & $(n, p)$ \\
\hline$I-128$ & ENDFb5/532dos & $I-127$ & $(n, g)$ & $\operatorname{Pr}-143$ & ENDFb 6 & $\mathrm{Nd}-145$ & $(n, t)$ \\
\hline$I-128$ & ENDL94 & $I-127$ & $(n, g)$ & $\operatorname{Pr}-144$ & ENDFb 6 & $\mathrm{Nd}-145$ & $(\mathrm{n}, \mathrm{np})$ \\
\hline$I-128$ & ENDFb 6 & $I-127$ & $(n, g)$ & $\operatorname{Pr}-144$ & ENDFb 6 & $\mathrm{Nd}-145$ & $(n, d)$ \\
\hline$I-128$ & ENDFb 6 & $\mathrm{Xe}-128$ & $(n, p)$ & $\operatorname{Pr}-144$ & ENDFb 6 & $\mathrm{Nd}-146$ & $(n, t)$ \\
\hline$I-128$ & ENDFb 6 & $\mathrm{Xe}-129$ & $(n, d)$ & $\operatorname{Pr}-144$ & ENDFb 6 & $\mathrm{Pm}-147$ & $(n, a)$ \\
\hline$I-128$ & ENDFb 6 & $\mathrm{Xe}-130$ & $(n, t)$ & $\operatorname{Pr}-145$ & ENDFb 6 & $\mathrm{Nd}-146$ & $(n, n p)$ \\
\hline$I-129$ & ENDFb 6 & $\mathrm{Xe}-129$ & $(n, p)$ & $\operatorname{Pr}-145$ & ENDFb 6 & $\mathrm{Nd}-145$ & $(n, p)$ \\
\hline$I-129$ & ENDFb 6 & $\mathrm{Xe}-130$ & $(n, d)$ & $\operatorname{Pr}-145$ & ENDFb 6 & $\mathrm{Nd}-146$ & $(n, d)$ \\
\hline$I-129$ & ENDFb 6 & $\mathrm{Xe}-131$ & $(n, t)$ & $\operatorname{Pr}-145$ & ENDFb 6 & $\mathrm{Pm}-147$ & $(\mathrm{n}, 3 \mathrm{He})$ \\
\hline$I-130$ & ENDFb 6 & $\mathrm{Xe}-130$ & $(n, p)$ & $\operatorname{Pr}-146$ & ENDFb 6 & $\mathrm{Nd}-146$ & $(n, p)$ \\
\hline$I-130$ & ENDFb 6 & $\mathrm{Xe}-131$ & $(n, d)$ & Pr -146 & ENDFb 6 & $\mathrm{Nd}-148$ & $(n, t)$ \\
\hline$I-130$ & ENDFb 6 & $\mathrm{Xe}-132$ & $(n, t)$ & $\operatorname{Pr}-147$ & ENDFb 6 & $\mathrm{Nd}-148$ & $(\mathrm{n}, \mathrm{np})$ \\
\hline$I-130$ & ENDFb 6 & $\mathrm{Cs}-133$ & $(n, a)$ & $\operatorname{Pr}-147$ & ENDFb 6 & $\mathrm{Nd}-148$ & $(\mathrm{n}, \mathrm{d})$ \\
\hline$I-131$ & ENDFb 6 & $\mathrm{Xe}-131$ & $(\mathrm{n}, \mathrm{p})$ & Pr -148 & ENDFb 6 & $\mathrm{Nd}-148$ & $(\mathrm{n}, \mathrm{p})$ \\
\hline$I-131$ & ENDFb 6 & $\mathrm{Xe}-132$ & $(n, d)$ & $\operatorname{Pr}-148$ & ENDFb 6 & $\mathrm{Nd}-150$ & $(n, t)$ \\
\hline$I-132$ & ENDFb 6 & $\mathrm{Xe}-132$ & $(n, p)$ & $\operatorname{Pr}-149$ & ENDFb 6 & $\mathrm{Nd}-150$ & $(n, n p)$ \\
\hline$I-132$ & ENDFb 6 & $\mathrm{Xe}-134$ & $(n, t)$ & Pr-149 & ENDFb 6 & $\mathrm{Nd}-150$ & $(n, d)$ \\
\hline$I-133$ & ENDFb 6 & $\mathrm{Xe}-134$ & $(n, d)$ & $\operatorname{Pr}-150$ & ENDFb 6 & $\mathrm{Nd}-150$ & $(n, p)$ \\
\hline$I-134$ & ENDFb 6 & $\mathrm{Xe}-134$ & $(n, p)$ & $\mathrm{Nd}-141$ & LLNL/ACTL & $\mathrm{Nd}-142$ & $(n, 2 n)$ \\
\hline$I-134$ & ENDFb 6 & $\mathrm{Xe}-136$ & $(n, t)$ & $\mathrm{Nd}-141$ & ENDFb 6 & $\mathrm{Nd}-143$ & $(n, 3 n)$ \\
\hline$I-135$ & ENDFb 6 & $\mathrm{Xe}-136$ & $(n, d)$ & $\mathrm{Nd}-144$ & ENDFb 6 & $\mathrm{Nd}-145$ & $(n, 2 n)$ \\
\hline$I-136$ & ENDFb 6 & $\mathrm{Xe}-136$ & $(n, p)$ & $\mathrm{Nd}-144$ & ENDFb 6 & $\mathrm{Nd}-146$ & $(n, 3 n)$ \\
\hline $\mathrm{Xe}-122$ & ENDFb 6 & $\mathrm{Xe}-124$ & $(n, 3 n)$ & $\mathrm{Nd}-144$ & ENDFb 6 & $\mathrm{Nd}-143$ & $(n, g)$ \\
\hline $\mathrm{xe}-123$ & ENDFb 6 & $\mathrm{Xe}-124$ & $(n, 2 n)$ & $\mathrm{Nd}-147$ & LLNL/ACTL & $\mathrm{Nd}-148$ & $(n, 2 n)$ \\
\hline $\mathrm{Xe}-125$ & ENDFb 6 & $\mathrm{Xe}-126$ & $(n, 2 n)$ & $\mathrm{Nd}-147$ & ENDFb 6 & $\mathrm{Nd}-148$ & $(n, 2 n)$ \\
\hline $\mathrm{Xe}-125$ & ENDFb 6 & $\mathrm{Xe}-124$ & $(n, g)$ & $\mathrm{Nd}-147$ & ENDFb 6 & $\mathrm{Sm}-151$ & $(\mathrm{n}, \mathrm{na})$ \\
\hline $\mathrm{Xe}-127$ & ENDFb 6 & $\mathrm{Xe}-128$ & $(n, 2 n)$ & $\mathrm{Nd}-147$ & ENDFb 6 & $\mathrm{Nd}-146$ & $(n, g)$ \\
\hline $\mathrm{Xe}-127$ & ENDFb 6 & $\mathrm{Xe}-129$ & $(n, 3 n)$ & $\mathrm{Nd}-147$ & ENDFb 6 & $P m-147$ & $(n, p)$ \\
\hline $\mathrm{Xe}-127$ & ENDFb 6 & $\mathrm{Xe}-126$ & $(n, g)$ & $\mathrm{Nd}-149$ & LLNL/ACTL & $\mathrm{Nd}-150$ & $(n, 2 n)$ \\
\hline $\mathrm{Xe}-133$ & ENDL94 & $\mathrm{Xe}-134$ & $(n, 2 n)$ & $\mathrm{Nd}-149$ & ENDFb 6 & $\mathrm{Nd}-150$ & $(n, 2 n)$ \\
\hline$x e-133$ & ENDFb 6 & $\mathrm{Xe}-134$ & $(n, 2 n)$ & $\mathrm{Nd}-149$ & ENDFb 6 & $\mathrm{Nd}-148$ & $(n, g)$ \\
\hline $\mathrm{Xe}-133$ & ENDFb 6 & $\mathrm{Xe}-132$ & $(n, g)$ & $\mathrm{Nd}-149$ & ENDFb 6 & $\mathrm{Sm}-151$ & $(\mathrm{n}, 3 \mathrm{He})$ \\
\hline $\mathrm{Xe}-133$ & ENDFb 6 & $\mathrm{Cs}-133$ & $(n, p)$ & $\mathrm{Nd}-151$ & ENDFb 6 & $\mathrm{Nd}-150$ & $(n, g)$ \\
\hline $\mathrm{Xe}-135$ & ENDFb 6 & $\mathrm{Xe}-136$ & $(n, 2 n)$ & $\mathrm{Pm}-145$ & ENDFb 6 & $\mathrm{Pm}-147$ & $(n, 3 n)$ \\
\hline $\mathrm{Xe}-135$ & ENDL94 & $\mathrm{Xe}-134$ & $(n, g)$ & $\mathrm{Pm}-146$ & ENDFb 6 & $P m-147$ & $(n, 2 n)$ \\
\hline $\mathrm{Xe}-135$ & ENDFb 6 & $\mathrm{Xe}-134$ & $(n, g)$ & $P m-148$ & ENDFb 6 & Eu-152 & $(n, n a)$ \\
\hline $\mathrm{Xe}-135$ & ENDFb 6 & $\mathrm{Ba}-138$ & $(n, a)$ & $\mathrm{Pm}-148$ & ENDFb 6 & $\mathrm{Pm}-147$ & $(n, g)$ \\
\hline $\mathrm{Xe}-137$ & ENDFb 6 & $\mathrm{Xe}-136$ & $(n, g)$ & Pm-149 & ENDFb 6 & $P m-148$ & $(n, g)$ \\
\hline $\mathrm{Cs}-132$ & LLNL/ACTL & $\mathrm{Cs}-133$ & $(n, 2 n)$ & $\mathrm{Pm}-149$ & ENDFb 6 & $\mathrm{Pm}-148$ & $(n, g)$ \\
\hline $\mathrm{Cs}-132$ & ENDFb 6 & $\mathrm{Cs}-133$ & $(n, 2 n)$ & $\mathrm{Pm}-149$ & ENDFb 6 & $\mathrm{Sm}-151$ & $(n, t)$ \\
\hline $\mathrm{Cs}-134$ & ENDFb 6 & $\mathrm{Cs}-133$ & $(n, g)$ & Pm-149 & ENDFb 6 & Eu-152 & $(n, a)$ \\
\hline $\mathrm{Cs}-138$ & ENDFb 6 & $\mathrm{Ba}-138$ & $(n, p)$ & $P m-150$ & ENDFb 6 & Eu-154 & $(\mathrm{n}, \mathrm{na})$ \\
\hline $\mathrm{Ba}-133$ & ENDFb 6 & $\mathrm{Ba}-134$ & $(n, 2 n)$ & $\mathrm{Pm}-150$ & ENDFb 6 & $\mathrm{Sm}-151$ & $(n, n p)$ \\
\hline $\mathrm{Ba}-139$ & ENDFb 6 & $\mathrm{Ba}-138$ & $(n, g)$ & $\mathrm{Pm}-150$ & ENDFb 6 & $\mathrm{Pm}-149$ & $(n, g)$ \\
\hline $\mathrm{La}-137$ & ENDFb 6 & Pr-141 & $(n, n a)$ & $P m-150$ & ENDFb 6 & $\mathrm{Sm}-151$ & $(n, d)$ \\
\hline $\mathrm{La}-140$ & ENDFb5/532dos & $\mathrm{La}-139$ & $(n, g)$ & $\mathrm{Pm}-150$ & ENDFb 6 & $\mathrm{Eu}-152$ & $(\mathrm{n}, 3 \mathrm{He})$ \\
\hline $\mathrm{La}-140$ & ENDFb 6 & $\mathrm{La}-139$ & $(n, g)$ & Pm-151 & ENDFb 6 & $\mathrm{Eu}-155$ & $(n, n a)$ \\
\hline $\mathrm{Ce}-139$ & LLNL/ACTL & $\mathrm{Ce}-140$ & $(n, 2 n)$ & Pm-151 & ENDFb 6 & $\mathrm{Sm}-151$ & $(n, p)$ \\
\hline $\mathrm{Ce}-139$ & ENDFb 6 & $\mathrm{Nd}-143$ & $(n, n a)$ & Pm-151 & ENDFb 6 & $\mathrm{Eu}-154$ & $(n, a)$ \\
\hline $\mathrm{Ce}-139$ & ENDFb 6 & Pr-141 & $(n, t)$ & $P m-152$ & ENDFb 6 & Eu-154 & $(\mathrm{n}, 3 \mathrm{He})$ \\
\hline $\mathrm{Ce}-141$ & LLNL/ACTL & $\mathrm{Ce}-142$ & $(n, 2 n)$ & $\mathrm{Pm}-152$ & ENDFb 6 & $\mathrm{Eu}-155$ & $(n, a)$ \\
\hline $\mathrm{Ce}-141$ & ENDFb 6 & $\mathrm{Nd}-145$ & $(n, n a)$ & $P m-153$ & ENDFb 6 & $\mathrm{Eu}-155$ & $(\mathrm{n}, 3 \mathrm{He})$ \\
\hline $\mathrm{Ce}-141$ & ENDFb 6 & $\mathrm{Ce}-140$ & $(n, g)$ & Sm-151 & ENDFb 6 & Eu-152 & $(n, n p)$ \\
\hline $\mathrm{Ce}-141$ & ENDFb 6 & Pr-141 & $(n, p)$ & Sm-151 & ENDFb 6 & Eu-152 & $(n, d)$ \\
\hline $\mathrm{Ce}-141$ & ENDFb 6 & $\mathrm{Nd}-143$ & $(\mathrm{n}, 3 \mathrm{He})$ & $\mathrm{Sm}-153$ & ENDFb 6 & $\mathrm{Eu}-154$ & $(n, n p)$ \\
\hline $\mathrm{Ce}-143$ & ENDFb 6 & $\mathrm{Ce}-142$ & $(n, g)$ & $\mathrm{Sm}-153$ & ENDFb 6 & $\mathrm{Eu}-154$ & $(n, d)$ \\
\hline $\mathrm{Ce}-143$ & ENDFb 6 & $\mathrm{Nd}-145$ & $(\mathrm{n}, 3 \mathrm{He})$ & $\mathrm{Sm}-153$ & ENDFb 6 & $\mathrm{Eu}-155$ & $(n, t)$ \\
\hline $\mathrm{Ce}-143$ & ENDFb 6 & $\mathrm{Nd}-146$ & $(n, a)$ & Sm-155 & ENDFb 6 & $\mathrm{Eu}-155$ & $(n, p)$ \\
\hline $\mathrm{Ce}-144$ & ENDFb 6 & $\mathrm{Nd}-148$ & $(n, n a)$ & $\mathrm{Eu}-150$ & ENDFb 6 & Eu-152 & $(n, 3 n)$ \\
\hline $\mathrm{Ce}-144$ & ENDFb 6 & $\mathrm{Nd}-146$ & $(\mathrm{n}, 3 \mathrm{He})$ & $\mathrm{Eu}-152$ & ENDFb 6 & Eu-154 & $(n, 3 n)$ \\
\hline $\mathrm{Ce}-145$ & ENDFb 6 & $\mathrm{Nd}-148$ & $(n, a)$ & $\mathrm{Eu}-154$ & ENDFb 6 & $\mathrm{Eu}-155$ & $(n, 2 n)$ \\
\hline $\mathrm{Ce}-146$ & ENDFb 6 & $\mathrm{Nd}-150$ & $(\mathrm{n}, \mathrm{na})$ & $\mathrm{Eu}-154$ & ENDL 94 & Eu-156 & $(n, 3 n)$ \\
\hline $\mathrm{Ce}-146$ & ENDFb 6 & $\mathrm{Nd}-148$ & $(\mathrm{n}, 3 \mathrm{He})$ & $\mathrm{Eu}-155$ & ENDL 94 & $\mathrm{Eu}-156$ & $(n, 2 n)$ \\
\hline $\mathrm{Ce}-147$ & ENDFb 6 & $\mathrm{Nd}-150$ & $(n, a)$ & $\mathrm{Eu}-155$ & ENDFb 6 & $\mathrm{Eu}-154$ & $(n, g)$ \\
\hline $\operatorname{Pr}-139$ & ENDFb 6 & Pr-141 & $(n, 3 n)$ & $\mathrm{Eu}-156$ & ENDFb 6 & $\mathrm{Eu}-155$ & $(n, g)$ \\
\hline
\end{tabular}


UCRL-TR-203529

\begin{tabular}{|c|c|c|c|c|c|c|c|}
\hline Eu-157 & ENDL 94 & $\mathrm{Eu}-156$ & $(n, g)$ & Ta-184 & ENDFb 6 & $\mathrm{~W}-184$ & $(n, p)$ \\
\hline $\mathrm{Eu}-157$ & ENDFb 6 & Eu-156 & $(n, g)$ & $\mathrm{Ta}-185$ & ENDFb 6 & $W-186$ & $(n, n p)$ \\
\hline $\mathrm{Eu}-158$ & ENDFb 6 & $\mathrm{Eu}-157$ & $(n, g)$ & Ta-186 & LLNL/ACTL & $\mathrm{W}-186$ & $(n, p)$ \\
\hline $\mathrm{Tb}-161$ & ENDFb 6 & $\mathrm{~Tb}-160$ & $(n, g)$ & $\mathrm{Ta}-186$ & ENDFb 6 & $W-186$ & $(n, p)$ \\
\hline Dy-166 & LLNL/ACTL & Но-166 & $(n, p)$ & W-178 & LLNL/ACTL & $W-180$ & $(n, 3 n)$ \\
\hline Ho -162 & LLNL/ACTL & Ho-164 & $(n, 3 n)$ & W-179 & LLNL/ACTL & $W-180$ & $(n, 2 n)$ \\
\hline Ho-163 & LLNL/ACTL & Ho-164 & $(n, 2 n)$ & $W-181$ & LLNL/ACTL & $\mathrm{W}-182$ & $(n, 2 n)$ \\
\hline Ho-164 & LLNL/ACTL & Ho-166 & $(n, 3 n)$ & W-181 & ENDFb 6 & $\mathrm{~W}-182$ & $(n, 2 n)$ \\
\hline Ho-167 & LLNL/ACTL & Ho-166 & $(n, g)$ & W-181 & LLNL/ACTL & $W-183$ & $(n, 3 n)$ \\
\hline $\mathrm{Tm}-168$ & LLNL/ACTL & $\mathrm{Tm}-169$ & $(n, 2 n)$ & W-181 & ENDFb 6 & $\mathrm{~W}-183$ & $(n, 3 n)$ \\
\hline $\mathrm{Tm}-170$ & LLNL/ACTL & $\mathrm{Tm}-169$ & $(n, g)$ & W-181 & LLNL/ACTL & $\mathrm{W}-180$ & $(n, g)$ \\
\hline $\mathrm{Tm}-172$ & ENDFb 6 & Lu-175 & $(n, a)$ & $W-185$ & LLNL/ACTL & $W-186$ & $(n, 2 n)$ \\
\hline $\mathrm{Tm}-173$ & ENDFb 6 & Lu-176 & $(n, a)$ & W-185 & ENDFb 6 & $\mathrm{~W}-186$ & $(n, 2 n)$ \\
\hline$Y b-175$ & ENDFb 6 & $\mathrm{Lu}-175$ & $(n, p)$ & $W-185$ & LLNL/ACTL & $W-184$ & $(n, g)$ \\
\hline $\mathrm{Lu}-173$ & LLNL/ACTL & Lu-175 & $(n, 3 n)$ & W-185 & ENDFb 6 & $\mathrm{~W}-184$ & $(n, g)$ \\
\hline $\mathrm{Lu}-173$ & ENDFb 6 & $\mathrm{Lu}-175$ & $(n, 3 n)$ & W-185 & LLNL/ACTL & $R e-185$ & $(n, p)$ \\
\hline Lu-174 & LLNL/ACTL & Lu-175 & $(n, 2 n)$ & W-187 & LLNL/ACTL & W-186 & $(n, g)$ \\
\hline $\mathrm{Lu}-174$ & ENDFb 6 & $\mathrm{Lu}-175$ & $(n, 2 n)$ & W-187 & ENDFb 6 & W-186 & $(n, g)$ \\
\hline Lu-174 & LLNL/ACTL & Lu-176 & $(n, 3 n)$ & W-187 & LLNL/ACTL & $R e-187$ & $(n, p)$ \\
\hline $\mathrm{Lu}-174$ & ENDFb 6 & $\mathrm{Lu}-176$ & $(n, 3 n)$ & $R e-183$ & LLNL/ACTL & $R e-185$ & $(n, 3 n)$ \\
\hline $\mathrm{Lu}-174$ & LLNL/ACTL & Hf -174 & $(n, p)$ & $R e-183$ & ENDL94 & $R e-185$ & $(n, 3 n)$ \\
\hline Lu-176 & LLNL/ACTL & Lu-175 & $(n, g)$ & $R e-183$ & ENDFb 6 & $R e-185$ & $(n, 3 n)$ \\
\hline $\mathrm{Lu}-176$ & ENDFb 6 & $\mathrm{Lu}-175$ & $(n, g)$ & $R e-184$ & LLNL/ACTL & $R e-185$ & $(n, 2 n)$ \\
\hline Lu-176 & LLNL/ACTL & $\mathrm{Hf}-176$ & $(\mathrm{n}, \mathrm{p})$ & $R e-184$ & ENDL 94 & $R e-185$ & $(n, 2 n)$ \\
\hline $\mathrm{Lu}-177$ & LLNL/ACTL & $\mathrm{Ta}-181$ & $(\mathrm{n}, \mathrm{na})$ & $R e-184$ & ENDFb 6 & $R e-185$ & $(n, 2 n)$ \\
\hline $\mathrm{Lu}-177$ & LLNL/ACTL & $\mathrm{Lu}-176$ & $(n, g)$ & $R e-186$ & LLNL/ACTL & $R e-187$ & $(n, 2 n)$ \\
\hline $\mathrm{Lu}-177$ & ENDFb 6 & $\mathrm{Lu}-176$ & $(n, g)$ & $R e-186$ & ENDL94 & $R e-187$ & $(n, 2 n)$ \\
\hline $\mathrm{Lu}-177$ & LLNL/ACTL & Hf -177 & $(n, p)$ & $R e-186$ & ENDFb 6 & $R e-187$ & $(n, 2 n)$ \\
\hline Lu-177 & LLNL/ACTL & $\mathrm{Ta}-180$ & $(n, a)$ & $R e-186$ & LLNL/ACTL & $R e-185$ & $(n, g)$ \\
\hline $\mathrm{Lu}-178$ & LLNL/ACTL & $\mathrm{Hf}-178$ & $(n, p)$ & $R e-186$ & ENDL 94 & $R e-185$ & $(n, g)$ \\
\hline $\mathrm{Lu}-179$ & LLNL/ACTL & Hf-179 & $(\mathrm{n}, \mathrm{p})$ & $R e-186$ & ENDFb 6 & $R e-185$ & $(n, g)$ \\
\hline $\mathrm{Lu}-180$ & LLNL/ACTL & $\mathrm{Hf}-180$ & $(n, p)$ & $R e-188$ & LLNL/ACTL & $R e-187$ & $(n, g)$ \\
\hline Hf -172 & LLNL/ACTL & $\mathrm{Hf}-174$ & $(n, 3 n)$ & $\mathrm{Re}-188$ & ENDL94 & $\mathrm{Re}-187$ & $(n, g)$ \\
\hline $\mathrm{Hf}-173$ & LLNL/ACTL & $\mathrm{Hf}-174$ & $(n, 2 n)$ & $R e-188$ & ENDFb 6 & $\mathrm{Re}-187$ & $(n, g)$ \\
\hline Hf -173 & ENDFb 6 & $\mathrm{Hf}-174$ & $(n, 2 n)$ & $\operatorname{Ir}-190$ & LLNL/ACTL & Ir-191 & $(n, 2 n)$ \\
\hline Hf -174 & LLNL/ACTL & $\mathrm{Hf}-176$ & $(n, 3 n)$ & Ir -190 & ENDFb 6 & $\operatorname{Ir}-191$ & $(n, 2 n)$ \\
\hline Hf -175 & LLNL/ACTL & Hf -176 & $(n, 2 n)$ & Ir -190 & LLNL/ACTL & Pt -190 & $(n, p)$ \\
\hline Hf -175 & ENDFb 6 & Hf -176 & $(n, 2 n)$ & Ir -192 & LLNL/ACTL & Ir-193 & $(n, 2 n)$ \\
\hline Hf -175 & LLNL/ACTL & $\mathrm{Hf}-177$ & $(n, 3 n)$ & Ir-192 & ENDFb 6 & Ir-193 & $(n, 2 n)$ \\
\hline Hf -181 & LLNL/ACTL & $\mathrm{Hf}-180$ & $(n, g)$ & Ir -192 & LLNL/ACTL & Pt-192 & $(n, p)$ \\
\hline Hf -181 & LLNL/ACTL & $\mathrm{Ta}-181$ & $(n, p)$ & Ir-194 & LLNL/ACTL & Pt-194 & $(n, p)$ \\
\hline Hf -181 & ENDFb 6 & $\mathrm{Ta}-181$ & $(n, p)$ & Ir-194 & LLNL/ACTL & $\mathrm{Au}-197$ & $(n, a)$ \\
\hline Hf -181 & LLNL/ACTL & $\mathrm{W}-184$ & $(n, a)$ & Ir -194 & ENDFb 6 & $\mathrm{Au}-197$ & $(n, a)$ \\
\hline Hf -181 & ENDFb 6 & $W-184$ & $(n, a)$ & Ir-195 & LLNL/ACTL & Pt-195 & $(n, p)$ \\
\hline Hf -182 & LLNL/ACTL & $\mathrm{W}-186$ & $(\mathrm{n}, \mathrm{na})$ & Ir-196 & LLNL/ACTL & Pt -196 & $(n, p)$ \\
\hline Hf -183 & LLNL/ACTL & $\mathrm{W}-186$ & $(n, a)$ & Ir -198 & LLNL/ACTL & Pt -198 & $(n, p)$ \\
\hline Hf -183 & ENDFb 6 & $\mathrm{~W}-186$ & $(n, a)$ & Pt -188 & LLNL/ACTL & Pt -190 & $(n, 3 n)$ \\
\hline $\mathrm{Ta}-178$ & LLNL/ACTL & $\mathrm{Ta}-180$ & $(n, 3 n)$ & Pt -189 & LLNL/ACTL & Pt -190 & $(n, 2 n)$ \\
\hline Ta-179 & LLNL/ACTL & $\mathrm{Ta}-180$ & $(n, 2 n)$ & Pt -190 & LLNL/ACTL & Pt-192 & $(n, 3 n)$ \\
\hline Ta-179 & LLNL/ACTL & $\mathrm{Ta}-181$ & $(n, 3 n)$ & Pt-191 & LLNL/ACTL & Pt-192 & $(n, 2 n)$ \\
\hline Ta-179 & ENDL 94 & $\mathrm{Ta}-181$ & $(n, 3 n)$ & Pt -193 & LLNL/ACTL & Pt-194 & $(n, 2 n)$ \\
\hline $\mathrm{Ta}-179$ & ENDFb 6 & $\mathrm{Ta}-181$ & $(n, 3 n)$ & Pt -193 & LLNL/ACTL & Pt -195 & $(n, 3 n)$ \\
\hline Ta-179 & LLNL/ACTL & $\mathrm{W}-180$ & $(n, n p)$ & Pt-197 & LLNL/ACTL & Pt -198 & $(n, 2 n)$ \\
\hline Ta-179 & LLNL/ACTL & $W-180$ & $(n, d)$ & Pt -197 & LLNL/ACTL & $\mathrm{Au}-197$ & $(n, p)$ \\
\hline $\mathrm{Ta}-180$ & LLNL/ACTL & $\mathrm{Ta}-181$ & $(n, 2 n)$ & Pt -197 & ENDFb 6 & $\mathrm{Au}-197$ & $(n, p)$ \\
\hline $\mathrm{Ta}-180$ & ENDL94 & $\mathrm{Ta}-181$ & $(n, 2 n)$ & Pt -199 & LLNL/ACTL & $\mathrm{Hg}-202$ & $(n, a)$ \\
\hline $\mathrm{Ta}-180$ & ENDFb 6 & $\mathrm{Ta}-181$ & $(n, 2 n)$ & Pt -201 & LLNL/ACTL & $\mathrm{Hg}-204$ & $(n, a)$ \\
\hline $\mathrm{Ta}-180$ & LLNL/ACTL & $\mathrm{W}-180$ & $(n, p)$ & $\mathrm{Au}-195$ & LLNL/ACTL & $\mathrm{Au}-197$ & $(n, 3 n)$ \\
\hline $\mathrm{Ta}-182$ & LLNL/ACTL & $\mathrm{W}-183$ & $(n, n p)$ & $\mathrm{Au}-195$ & ENDFb 6 & $\mathrm{Au}-197$ & $(n, 3 n)$ \\
\hline $\mathrm{Ta}-182$ & ENDFb 6 & $\mathrm{~W}-183$ & $(\mathrm{n}, \mathrm{np})$ & $\mathrm{Au}-196$ & LLNL/ACTL & $\mathrm{Au}-197$ & $(n, 2 n)$ \\
\hline $\mathrm{Ta}-182$ & LLNL/ACTL & $\mathrm{Ta}-181$ & $(n, g)$ & $\mathrm{Au}-196$ & ENDFb 6 & $\mathrm{Au}-197$ & $(n, 2 n)$ \\
\hline $\mathrm{Ta}-182$ & ENDL94 & $\mathrm{Ta}-181$ & $(n, g)$ & $\mathrm{Au}-198$ & LLNL/ACTL & $\mathrm{Au}-197$ & $(n, g)$ \\
\hline $\mathrm{Ta}-182$ & ENDFb 6 & $\mathrm{Ta}-181$ & $(n, g)$ & $\mathrm{Au}-198$ & ENDFb 6 & $\mathrm{Au}-197$ & $(n, g)$ \\
\hline $\mathrm{Ta}-182$ & LLNL/ACTL & $\mathrm{W}-182$ & $(n, p)$ & $\mathrm{Au}-201$ & LLNL/ACTL & $\mathrm{Hg}-202$ & $(n, n p)$ \\
\hline $\mathrm{Ta}-182$ & ENDFb 6 & $W-182$ & $(n, p)$ & $\mathrm{Au}-202$ & LLNL/ACTL & $\mathrm{Hg}-202$ & $(n, p)$ \\
\hline $\mathrm{Ta}-182$ & LLNL/ACTL & $\mathrm{W}-183$ & $(n, d)$ & $\mathrm{Au}-203$ & LLNL/ACTL & $\mathrm{Hg}-204$ & $(n, n p)$ \\
\hline $\mathrm{Ta}-183$ & LLNL/ACTL & $\mathrm{W}-184$ & $(n, n p)$ & $\mathrm{Au}-204$ & LLNL/ACTL & $\mathrm{Hg}-204$ & $(n, p)$ \\
\hline $\mathrm{Ta}-183$ & ENDFb 6 & $\mathrm{~W}-184$ & $(n, n p)$ & $\mathrm{Hg}-203$ & LLNL/ACTL & $\mathrm{Hg}-204$ & $(n, 2 n)$ \\
\hline $\mathrm{Ta}-183$ & LLNL/ACTL & $W-183$ & $(n, p)$ & $\mathrm{Hg}-203$ & LLNL/ACTL & $\mathrm{Pb}-207$ & $(n, n a)$ \\
\hline $\mathrm{Ta}-183$ & ENDFb 6 & W-183 & $(n, p)$ & $\mathrm{Hg}-203$ & ENDFb 6 & $\mathrm{~Pb}-208$ & $(\mathrm{n}, 2 \mathrm{na})$ \\
\hline $\mathrm{Ta}-183$ & LLNL/ACTL & $W-184$ & $(n, d)$ & $\mathrm{Hg}-203$ & LLNL/ACTL & $\mathrm{Hg}-202$ & $(n, g)$ \\
\hline $\mathrm{Ta}-184$ & LLNL/ACTL & $W-184$ & $(n, p)$ & $\mathrm{Hg}-203$ & LLNL/ACTL & Tl-203 & $(n, p)$ \\
\hline
\end{tabular}


UCRL-TR-203529

\begin{tabular}{|llll}
$\mathrm{Hg}-203$ & LLNL/ACTL & $\mathrm{Pb}-206$ & $(\mathrm{n}, \mathrm{a})$ \\
$\mathrm{Hg}-203$ & ENDFb & $\mathrm{Pb}-206$ & $(\mathrm{n}, \mathrm{a})$ \\
$\mathrm{Hg}-205$ & LLNL/ACTL & $\mathrm{Hg}-204$ & $(\mathrm{n}, \mathrm{g})$ \\
$\mathrm{Hg}-205$ & LLNL/ACTL & $\mathrm{Tl}-205$ & $(\mathrm{n}, \mathrm{p})$ \\
$\mathrm{Tl}-201$ & LLNL/ACTL & $\mathrm{Tl}-203$ & $(\mathrm{n}, 3 \mathrm{n})$ \\
$\mathrm{Tl}-202$ & LLNL/ACTL & $\mathrm{Tl}-203$ & $(\mathrm{n}, 2 \mathrm{n})$ \\
$\mathrm{Tl}-202$ & LLNL/ACTL & $\mathrm{Pb}-204$ & $(\mathrm{n}, \mathrm{t})$ \\
$\mathrm{Tl}-204$ & LLNL/ACTL & $\mathrm{Tl}-205$ & $(\mathrm{n}, 2 \mathrm{n})$ \\
$\mathrm{Tl}-204$ & LLNL/ACTL & $\mathrm{Tl}-203$ & $(\mathrm{n}, \mathrm{g})$ \\
$\mathrm{Tl}-204$ & LLNL/ACTL & $\mathrm{Pb}-204$ & $(\mathrm{n}, \mathrm{p})$ \\
$\mathrm{Tl}-204$ & LLNL/ACTL & $\mathrm{Pb}-206$ & $(\mathrm{n}, \mathrm{t})$ \\
$\mathrm{Tl}-204$ & ENDFb6 & $\mathrm{Pb}-206$ & $(\mathrm{n}, \mathrm{t})$ \\
$\mathrm{Tl}-206$ & ENDFb6 & $\mathrm{Pb}-208$ & $(\mathrm{n}, \mathrm{nd})$ \\
$\mathrm{Tl}-206$ & ENDFb6 & $\mathrm{Pb}-208$ & $(\mathrm{n}, 2 \mathrm{np})$ \\
$\mathrm{Tl}-206$ & LLNL/ACTL & $\mathrm{Tl}-205$ & $(\mathrm{n}, \mathrm{g})$ \\
$\mathrm{Tl}-206$ & LLNL/ACTL & $\mathrm{Pb}-206$ & $(\mathrm{n}, \mathrm{p})$ \\
$\mathrm{Tl}-206$ & ENDFb6 & $\mathrm{Pb}-206$ & $(\mathrm{n}, \mathrm{p})$ \\
$\mathrm{Tl}-206$ & LLNL/ACTL & $\mathrm{Bi}-209$ & $(\mathrm{n}, \mathrm{a})$ \\
$\mathrm{Tl}-207$ & ENDFb6 & $\mathrm{Pb}-208$ & $(\mathrm{n}, \mathrm{np})$ \\
$\mathrm{Tl}-207$ & LLNL/ACTL & $\mathrm{Pb}-207$ & $(\mathrm{n}, \mathrm{p})$ \\
$\mathrm{Tl}-207$ & ENDFb6 & $\mathrm{Pb}-207$ & $(\mathrm{n}, \mathrm{p})$ \\
$\mathrm{Pb}-204$ & LLNL/ACTL & $\mathrm{Pb}-206$ & $(\mathrm{n}, 3 \mathrm{n})$ \\
$\mathrm{Pb}-204$ & ENDFb6 & $\mathrm{Pb}-206$ & $(\mathrm{n}, 3 \mathrm{n})$ \\
$\mathrm{Pb}-205$ & LLNL/ACTL & $\mathrm{Pb}-206$ & $(\mathrm{n}, 2 \mathrm{n})$ \\
$\mathrm{Pb}-205$ & ENDFb6 & $\mathrm{Pb}-206$ & $(\mathrm{n}, 2 \mathrm{n})$ \\
$\mathrm{Pb}-205$ & LLNL/ACTL & $\mathrm{Pb}-207$ & $(\mathrm{n}, 3 \mathrm{n})$ \\
$\mathrm{Pb}-205$ & ENDFb6 & $\mathrm{Pb}-207$ & $(\mathrm{n}, 3 \mathrm{n})$ \\
$\mathrm{Pb}-205$ & LLNL/ACTL & $\mathrm{Pb}-204$ & $(\mathrm{n}, \mathrm{g})$ \\
$\mathrm{Pb}-209$ & LLNL/ACTL & $\mathrm{Pb}-208$ & $(\mathrm{n}, \mathrm{g})$ \\
$\mathrm{Pb}-209$ & LLNL/ACTL & $\mathrm{Bi}-209$ & $(\mathrm{n}, \mathrm{p})$ \\
$\mathrm{Bi}-207$ & LLNL/ACTL & $\mathrm{Bi}-209$ & $(\mathrm{n}, 3 \mathrm{n})$ \\
$\mathrm{Bi}-207$ & ENDL94 & $\mathrm{Bi}-209$ & $(\mathrm{n}, 3 \mathrm{n})$ \\
$\mathrm{Bi}-208$ & LLNL/ACTL & $\mathrm{Bi}-209$ & $(\mathrm{n}, 2 \mathrm{n})$ \\
$\mathrm{Bi}-208$ & ENDL94 & $\mathrm{Bi}-209$ & $(\mathrm{n}, 2 \mathrm{n})$ \\
$\mathrm{Bi}-210$ & LLNL/ACTL & $\mathrm{Bi}-209$ & $(\mathrm{n}, \mathrm{g})$ \\
$\mathrm{Bi}-210$ & ENDL94 & $\mathrm{Bi}-209$ & $(\mathrm{n}, \mathrm{g})$ \\
$\mathrm{Th}-229$ & LLNL/ACTL & $\mathrm{Th}-232$ & $(\mathrm{n}, 4 \mathrm{n})$ \\
$\mathrm{Th}-229$ & ENDL94 & $\mathrm{Th}-232$ & $(\mathrm{n}, 4 \mathrm{n})$ \\
$\mathrm{Th}-230$ & LLNL/ACTL & $\mathrm{Th}-232$ & $(\mathrm{n}, 3 \mathrm{n})$ \\
$\mathrm{TLN}$ & ENDFb6 & $\mathrm{Th}-232$ & $(\mathrm{n}, 3 \mathrm{n})$ \\
$\mathrm{Th}-232$ & $(\mathrm{n}, 3 \mathrm{n})$
\end{tabular}

\begin{tabular}{|c|c|c|c|}
\hline Th-231 & LLNL/ACTL & Th-232 & $(n, 2 n)$ \\
\hline Th-231 & ENDL 94 & $\mathrm{Th}-232$ & $(n, 2 n)$ \\
\hline Th-231 & ENDFb 6 & Th-232 & $(n, 2 n)$ \\
\hline Th-233 & LLNL/ACTL & Th -232 & $(n, g)$ \\
\hline Th-233 & ENDL 94 & Th -232 & $(n, g)$ \\
\hline Th-233 & ENDFb 6 & Th-232 & $(n, g)$ \\
\hline $\mathrm{Pa}-229$ & ENDFb 6 & $\mathrm{~Pa}-231$ & $(n, 3 n)$ \\
\hline $\mathrm{Pa}-230$ & ENDFb 6 & $\mathrm{~Pa}-231$ & $(\mathrm{n}, 2 \mathrm{n})$ \\
\hline $\mathrm{Pa}-232$ & ENDFb5/532dos & $\mathrm{Pa}-23 \mathrm{I}$ & $(n, g)$ \\
\hline $\mathrm{Pa}-232$ & ENDFb 6 & $\mathrm{~Pa}-231$ & $(n, g)$ \\
\hline $\mathrm{U}-232$ & LLNL/ACTL & $U-234$ & $(n, 3 n)$ \\
\hline $\mathrm{U}-232$ & ENDL 94 & $U-234$ & $(n, 3 n)$ \\
\hline $\mathrm{U}-232$ & ENDFb 6 & $\mathrm{U}-234$ & $(n, 3 n)$ \\
\hline $\mathrm{U}-232$ & LLNL/ACTL & $U-235$ & $(n, 4 n)$ \\
\hline $\mathrm{U}-232$ & ENDL 94 & $\mathrm{U}-235$ & $(n, 4 n)$ \\
\hline $\mathrm{U}-232$ & ENDFb 6 & $\mathrm{U}-235$ & $(n, 4 n)$ \\
\hline $\mathrm{U}-233$ & LLNL/ACTL & $\mathrm{U}-234$ & $(\mathrm{n}, 2 \mathrm{n})$ \\
\hline $\mathrm{U}-233$ & ENDL94 & $U-234$ & $(n, 2 n)$ \\
\hline $\mathrm{U}-233$ & ENDFb 6 & $\mathrm{U}-234$ & $(n, 2 n)$ \\
\hline $\mathrm{U}-233$ & LLNL/ACTL & $\mathrm{U}-235$ & $(n, 3 n)$ \\
\hline $\mathrm{U}-233$ & ENDL94 & $U-235$ & $(n, 3 n)$ \\
\hline $\mathrm{U}-233$ & ENDFb 6 & $\mathrm{U}-235$ & $(n, 3 n)$ \\
\hline $\mathrm{U}-234$ & LLNL/ACTL & $\mathrm{U}-235$ & $(n, 2 n)$ \\
\hline $\mathrm{U}-234$ & ENDL 94 & $U-235$ & $(n, 2 n)$ \\
\hline $\mathrm{U}-234$ & ENDFb 6 & $\mathrm{U}-235$ & $(n, 2 n)$ \\
\hline $\mathrm{U}-235$ & LLNL/ACTL & $U-238$ & $(n, 4 n)$ \\
\hline$U-235$ & ENDL 94 & $U-238$ & $(n, 4 n)$ \\
\hline $\mathrm{U}-235$ & ENDFb 6 & $U-238$ & $(n, 4 n)$ \\
\hline $\mathrm{U}-235$ & LLNL/ACTL & $\mathrm{U}-234$ & $(n, g)$ \\
\hline $\mathrm{U}-235$ & ENDL 94 & $U-234$ & $(n, g)$ \\
\hline $\mathrm{U}-235$ & ENDFb 6 & $\mathrm{U}-234$ & $(n, g)$ \\
\hline$U-236$ & LLNL/ACTL & $U-238$ & $(n, 3 n)$ \\
\hline$U-236$ & ENDL94 & $U-238$ & $(n, 3 n)$ \\
\hline $\mathrm{U}-236$ & ENDFb 6 & $\mathrm{U}-238$ & $(n, 3 n)$ \\
\hline$U-236$ & LLNL/ACTL & $\mathrm{U}-235$ & $(n, g)$ \\
\hline$U-236$ & ENDL94 & $U-235$ & $(n, g)$ \\
\hline$U-236$ & ENDFb 6 & $U-235$ & $(n, g)$ \\
\hline $\mathrm{U}-237$ & LLNL/ACTL & $\mathrm{U}-238$ & $(n, 2 n)$ \\
\hline $\mathrm{U}-237$ & ENDL 94 & $U-238$ & $(\mathrm{n}, 2 \mathrm{n})$ \\
\hline $\mathrm{U}-237$ & ENDFb 6 & $U-238$ & $(\mathrm{n}, 2 \mathrm{n})$ \\
\hline
\end{tabular}

